\title{
REGRESSÃO LOGÍSTICA PARCIAL : UMA TÉCNICA PARAMÉTRICA EM ANÁLISE DE SOBREVIVÊNCIA
}

\author{
Carine Savalli
}

\author{
DISSERTAÇÃO APRESENTADA AO \\ INSTITUTO DE MATEMÁTICA E ESTATÍSTICA \\ DA UNIVERSIDADE DE SÃO PAULO \\ PARA OBTENÇÃO DO GRAU DE \\ MESTRE EM ESTATÍSTICA \\ Área de concentração : Estatística \\ Orientador: Prof. Dr. Gilberto Alvarenga Paula
}

Durante o curso de Mestrado e elaboração desse trabalho

o autor recebeu apoio financeiro do $\mathrm{CNPq}$

- São Paulo, maio de 1998 - 


\section{REGRESSÃO LOGÍSTICA PARCIAL : UMA TÉCNICA PARAMÉTRICA EM ANÁLISE DE SOBREVIVÊNCIA}

Este exemplar corresponde à redação

final da dissertação devidamente corrigida

e defendida por Carine Savalli e aprovada

pela comissão julgadora.

São Paulo, 8 de maio de 1998.

Comissão Julgadora:

- Professor Dr. Gilberto Alvarenga Paula (Orientador) - IME-USP

- Professor Dr. Heleno Bolfarine - IME - USP

- Professor Dr. Enrico Colosimo - UFMG 


\section{Aos meus pais}

"Seus conhecimentos e experiências sobre a vida conduziram-me além das teorias e metodologias, fazendo-me alcançar mais esse objetivo" 


\section{Agradecimentos}

São muitas as pessoas às quais eu gostaria de agradecer, que de alguma forma, colaboraram para a realização desse trabalho.

Aos meus pais, pela confiança e constante incentivo, sem os quais não seria possível alcançar este objetivo, o meu eterno obrigado.

Sou grata em especial ao meu orientador, o Prof. Gilberto, pela excelente orientação e apoio durante todo o curso de Mestrado e desenvolvimento desta dissertação.

Devo também agradecer aos meus colegas de curso, assim como ao Fernando, presentes em todos os momentos. Pelo companheirismo, amizade e compreensão, obrigada.

Agradeço também às minhas colegas de trabalho, Rita, Dália e Mariana, que na reta final da redação desse trabalho muito me incentivaram. Obrigada em especial à Rita, por compreender a necessidade de me ausentar em algumas ocasiões no trabalho em função de reuniões com meu orientador.

Agradeço ainda aos professores deste Instituto pela oportunidade de melhorar meus conhecimentos e minha formação acadêmica. 


\section{RESUMO}

Uma técnica largamente utilizada para estimar a função de sobrevivência para dados censurados é a técnica não-paramétrica de Kaplan-Meier que, apesar de ser facilmente calculável e interpretável, apresenta algumas limitações e, em alguns casos, estimadores paramétricos devem ser considerados. Entretanto, a teoria paramétrica clássica, também apresenta limitações, uma vez que escolher uma distribuição para a função de sobrevivência implica em fixar a forma da função de risco. Este trabalho pretende apresentar uma técnica paramétrica alternativa: a Regressão Logística Parcial, que permite a modelagem da função de risco bem como da função de sobrevivência. Trata-se de uma técnica adequada para estudos em que se tem interesse em analisar não só o comportamento da função de sobrevivência, mas também da função de risco.

\section{ABSTRACT}

The non-parametric Kaplan-Meier technique is a widely used approach for estimating the survivor function with censored data. In spite of facilitates for calculation and interpretation, it presents some limitations, and in some cases, parametric estimators should be considered. However, the classic parametric theory also presents limitations, since choosing a distribution to the survivor function implies in fixing a form for the hazard function. This works intends to present a parametric alternative: the Partial Logistic Regression, which allows modeling the hazard function as well as the survivor function. This technique is adequate to researchs in which it is important to analyse not only the survivor function but also the behaviour of hazard function. 


\section{CONTEÚDO}

Introdução

1

Capítulo 1 - Conceitos básicos em análise de sobrevivência

1.1 Introdução

2

1.2 Métodos não-paramétricos para estimação da função de sobrevivência $\quad$.............................. 4

1.2.1 O estimador da tábua de vida padrão

1.2.2 O estimador de Kaplan-Meier

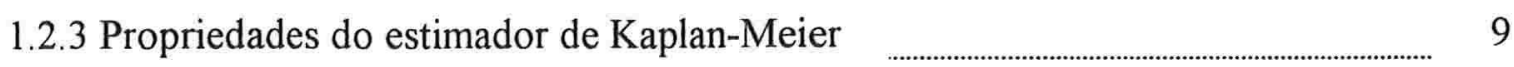

1.3 Comparação de duas curvas de sobrevivência $\quad$................................................................................. 10

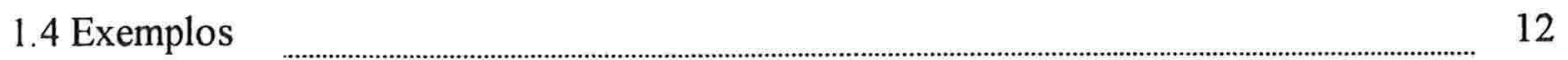

\section{Capítulo 2 - Modelo de regressão logística parcial}

2.1 Regressão Logística Parcial

2.1.1 Estimação da taxa de risco e função de sobrevivência 19

2.1.2 Estimação dos desvios-padrão da taxa de risco e função de sobrevivência 22

2.2 Função Desvio 26

2.3 Teste de hipóteses acerca do vetor de parâmetros $\alpha$ 28

2.4 Resíduos e técnicas de diagnósticos 32 
2.6 Paralelo entre estimação paramétrica e não-paramétrica

2.7 Relação entre o modelo condicional e não-condicional

2.80 caso contínuo

2.9 Como escolher a melhor discretização para o tempo

2.10 O exemplo do câncer na cabeça e pescoço

\section{Capítulo 3 - Aplicações}

3.1 Estudo sobre carreira de educadores de crianças excepcionais 75

3.2 Estudo seriado com ratos 82

3.3 Estudo sobre a sobrevivência a câncer no ovário após a segunda laparotomia 96

Conclusão

Apêndice A - Dados 109

Apêndice B - Programas no Splus 116

Referências Bibliográficas 126 


\section{INTRODUÇÃO}

Este trabalho está baseado no artigo "Logistic Regression, Survival Analysis, and the Kaplan-Meier Curve" de Bradley Efron, publicado em 1988 na revista Journal of the American Statistical Association, 83, 414-425, e na teoria de modelos lineares generalizados já bastante explorada por vários autores.

A proposta de Efron consiste em unir duas metodologias estatísticas aparentemente disjuntas, a análise de sobrevivência e a regressão logística, de tal forma a atingir o objetivo específico de estudar o comportamento da função de risco ao longo do tempo. A técnica em questão, denominada Regressão Logística Parcial, pode ser aplicada em dados com censuras e permite estimar a taxa de risco e a curva de sobrevivência bem como seus respectivos desviospadrão, além de consistir em uma alternativa à técnica de estimação não-paramétrica de Kaplan-Meier.

No Capítulo 1 serão apresentados alguns exemplos em que a técnica de Kaplan-Meier é frequentemente aplicada. Além disso, serão discutidas as implicações em se usar o estimador de Kaplan-Meier para analisar dados com censuras.

No Capítulo 2 será definido o modelo de Regressão Logística Parcial no contexto de análise de sobrevivência, sendo sempre que possível comparado à técnica não-paramétrica de Kaplan-Meier. O ajuste do modelo em questão será estudado através de técnicas de diagnóstico para modelos lineares generalizados.

Por fim, no Capítulo 3 o modelo de Regressão Logística Parcial será aplicado em problemas práticos e os resultados discutidos. 


\section{CAPÍTULO 1}

\section{CONCEITOS BÁSICOS EM ANÁLISE DE SOBREVIVÊNCIA}

\subsection{Introdução}

A análise de sobrevivência consiste em um conjunto de técnicas estatísticas paramétricas, não-paramétricas e gráficas adequadas a dados que medem o tempo até a ocorrência de um evento de interesse, como falha de um componente de uma máquina, morte de um paciente devido à uma certa doença, entre outros. Essa metodologia foi especialmente desenvolvida para incorporar a informação de observações censuradas, que são aquelas em que não foi possível observar a ocorrência do evento de interesse. O tempo exato de sobrevivência desses indivíduos é desconhecido, sabe-se somente que é maior que um certo valor. Essas censuras podem ocorrer em várias situações e de diversas formas: quando o tempo até a ocorrência do evento é maior que o tempo de estudo, quando um indivíduo é perdido ao longo do seguimento, quando o evento de interesse já havia ocorrido antes do início do estudo e em muitas outras situações. 
Devido à sua vasta área de aplicação, a análise de sobrevivência tem se tornado um tópico de considerável interesse entre os pesquisadores das áreas biomédicas e engenharias. As técnicas paramétricas e não-paramétricas são igualmente importantes, entretanto, as últimas apresentam a vantagem de não depender da suposição de uma distribuição conhecida para os tempos de sobrevivência, além de produzirem resultados de fácil interpretação.

Os tempos de sobrevivência são variáveis aleatórias e portanto possuem uma distribuição associada que pode ser descrita por três funções: função de sobrevivência, função densidade de probabilidade e função de risco. Essas três funções são matematicamente equivalentes mas ilustram aspectos diferentes dos dados.

Para efeito de padronização, será considerado sempre nesse texto o evento morte como sendo o evento de interesse. Considere, então, a variável aleatória não-negativa e absolutamente contínua $T$, indicando o tempo de sobrevivência. A função de sobrevivência, denotada por $G(t)$, é definida como sendo a probabilidade de um indivíduo sobreviver além do instante $t$ :

$$
G(t)=P(T>t)
$$

Na prática, quando não há observações censuradas, a função de sobrevivência é estimada pela proporção de indivíduos que sobreviveram além do instante $t$ com relação ao total de indivíduos no estudo, no entanto, na presença de censuras, estimadores mais gerais devem ser considerados. Essa função, além de ser não-crescente com o tempo, possui as seguintes propriedades:

$$
\begin{aligned}
& G(t)=1, \text { para } t=0 ; \\
& G(t)=0, \text { para } t=\infty .
\end{aligned}
$$

A função densidade de probabilidade de $T$ é definida pelo limite da probabilidade de morte, em um intervalo de tempo $\Delta t$ tendendo a zero: 


$$
f(t)=\lim _{\Delta t \rightarrow 0} \frac{P\{T \in[t, t+\Delta t)\}}{\Delta t} .
$$

Na prática, a função densidade de probabilidade é estimada pela proporção de indivíduos que morreram em um intervalo de tempo $\Delta t$. Essa função possui duas propriedades: é não-negativa e a área abaixo da curva por ela definida é igual a 1.

Por fim, a função de risco ou taxa de risco é definida pelo limite da probabilidade de morte em um intervalo de tempo $\Delta t$ tendendo a zero dado que o indivíduo sobreviveu até o início desse intervalo:

$$
h(t)=\lim _{\Delta t \rightarrow 0} \frac{P\{T \in[t, t+\Delta t) \mid T>t\}}{\Delta t} .
$$

Na prática, a função de risco é estimada pela proporção de indivíduos que morreram em um intervalo de tempo $\Delta t$ muito pequeno, com relação ao total de indivíduos que sobreviveram até o início do intervalo.

A função de risco, também conhecida como taxa instantânea de morte, informa sobre o risco de morte por unidade de tempo durante o processo. Essa função pode ser decrescente, crescente, constante ou um processo mais complexo. Em particular, $h(t) \Delta t$ é uma aproximação da probabilidade de morte no intervalo $[t, t+\Delta t)$, dado que o indivíduo sobreviveu até o instante $t$.

\subsection{Métodos não-paramétricos para estimação da função de sobrevivência}

Escolher uma distribuição teórica para o tempo de sobrevivência nem sempre é uma tarefa simples, mas a função de risco representa uma importante ferramenta na escolha do modelo mais adequado pois, uma vez determinada a distribuição do tempo de sobrevivência, a forma da função de risco ao longo do tempo fica pré-estabelecida. Portanto, através de uma 
avaliação da função de risco é possivel, em alguns casos, inferir sobre a distribuição do tempo de sobrevivência. Dentre as distribuições que merecem destaque por serem as mais utilizadas estão a exponêncial, Weibull e gama.

Os métodos não-paramétricos para a análise de dados de sobrevivência são menos eficientes que os paramétricos quando a distribuição do tempo de sobrevida é conhecida. Entretanto, na falta de informação sobre a forma dessa distribuição, constituem, juntamente com as técnicas gráficas, uma alternativa natural de estimação.

Das três funções apresentadas anteriormente, a função de sobrevivência e sua representação gráfica são largamente utilizadas, sendo importante, portanto, definir os estimadores usuais. Aqui serão apresentados dois estimadores não-paramétricos: o estimador da tábua de vida padrão e o estimador produto limite ou de Kaplan-Meier que corresponde ao caso limite do primeiro.

\subsubsection{O estimador da tábua de vida padrão}

O método de estimação da tábua de vida, um dos mais antigos para a análise de dados de sobrevivência, é essencialmente uma extensão das tabelas de frequências relativas para o caso em que existem observações censuradas. Com o intuito de introduzir a notação utilizada nas próximas seções, considere que $n$ indivíduos foram acompanhados por um certo período de tempo $[0, \Im]$ e que seus tempos de morte ou censura foram observados. Considere também que todo o período estudado tenha sido particionado em $k$ intervalos segundo as constantes 0 $=\varepsilon_{0}<\varepsilon_{l}<\ldots<\varepsilon_{k}=\mathfrak{I}$ como descrito na Figura1.1. Em cada intervalo $I_{i}=\left(\varepsilon_{i-1}, \varepsilon_{i}\right]$ observou-se as seguintes quantidades:

$n_{i}$ : número de indivíduos em risco no início do intervalo $I_{i}$, para $i=1, \ldots, k$;

$s_{i}$ : número de mortes no intervalo $I_{i}$, para $i=I, \ldots, k$;

$s_{i}{ }^{\prime}$ : número de censuras no intervalo $I_{i}$, para $i=1, \ldots, k$. 


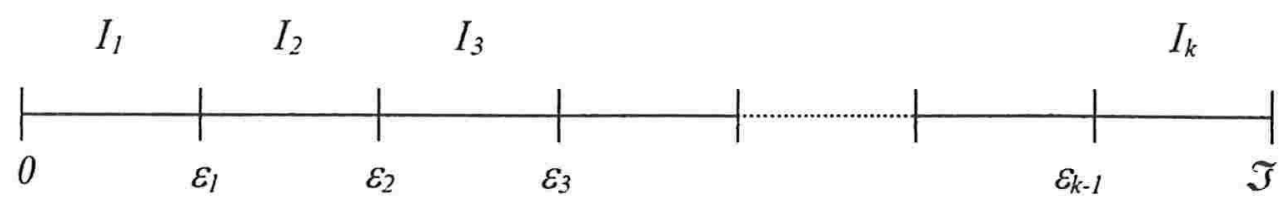

Figura 1.1: Representação do particionamento do período estudado em $k$ intervalos.

Note que, o número de indivíduos em risco no início do intervalo $I_{i}$ pode ser obtido através do simples cálculo

$$
n_{i}=n_{i-1}-s_{i-1}-s_{i-1}^{\prime} .
$$

Nesse contexto, a taxa de risco discreta é dada pela probabilidade condicional de morte no $i$-ésimo intervalo

$$
h_{i}=P\left\{T \leq \varepsilon_{i}: T>\varepsilon_{i-1}\right\},
$$

e portanto pelo teorema da probabilidade composta (ver DeGroot, 1989):

$$
\begin{aligned}
G\left(\varepsilon_{i}\right) & =P\left\{T>\varepsilon_{i}\right\} \\
& =P\left\{T>\varepsilon_{i} \mid T>\varepsilon_{i-1}\right\} P\left\{T>\varepsilon_{i-l} \mid T>\varepsilon_{i-2}\right\} \ldots P\left\{T>\varepsilon_{l} \mid T>\varepsilon_{0}\right\} P\left\{T>\varepsilon_{0}\right\} \\
& =\left(1-P\left\{T \leq \varepsilon_{i} \mid T>\varepsilon_{i-1}\right\}\right)\left(1-P\left\{T \leq \varepsilon_{i-1} \quad T>\varepsilon_{i-2}\right\}\right) \ldots\left(1-P\left\{T \leq \varepsilon_{l} \mid T>\varepsilon_{0}\right\}\right) \\
& =\left(1-h_{i}\right)\left(1-h_{i-1}\right) \ldots\left(1-h_{l}\right) \\
& =\prod_{l \leq j \leq i}\left(1-h_{j}\right) .
\end{aligned}
$$


Esse resultado é muito importante pois é a base para o método de estimação da tábua de vida padrão e do Kaplan-Meier. Ele indica que a probabilidade de sobrevivência no tempo $\varepsilon_{i}$ pode ser obtida através do produto das probabilidades condicionais de sobrevivência dos intervalos anteriores.

Assim, para o caso sem censura, o estimador da tábua de vida para a probabilidade condicional de morte no $i$-ésimo intervalo é simplesmente dado por

$$
\tilde{h}_{i}=\frac{s_{i}}{n_{i}}
$$

Se, entretanto, houver censura $\left(s_{i}{ }^{\prime} \geq 1\right)$, a quantidade em (1.1) deve estar subestimando a taxa de risco discreta, uma vez que é possível que alguns dos indivíduos censurados tenham permanecido em risco em somente parte desse intervalo. Nesse caso, o método da tábua de vida assume que as censuras tenham ocorrido de maneira uniforme ao longo do intervalo e que, em média, indivíduos censurados permanecem em risco durante metade desse intervalo. Considerando então essa correção, o estimador da tábua de vida padrão para a probabilidade condicional de morte no $i$-ésimo intervalo é aproximado por

$$
\tilde{h}_{i}=\frac{s_{i}}{n_{i}-\frac{l}{2} s_{i}{ }^{\prime}}=\frac{s_{i}}{n_{i}{ }^{\prime}},
$$

consequentemente, o estimador da tábua de vida padrão para a função de sobrevivência em um instante $t_{i}$ qualquer, dentro do período estudado, é dado por

$$
\widetilde{G}\left(t_{i}\right)=\prod_{I \leq j \leq i}\left(1-\frac{s_{j}}{n_{j}{ }^{\prime}}\right) .
$$


É possível mostrar (ver Lawless, 1982) que a variância aproximada do estimador da função de sobrevivência, para amostras grandes, é dada por

$$
\operatorname{Var}\left(\widetilde{G}\left(t_{i}\right)\right)=\widetilde{G}\left(t_{i}\right)^{2} \sum_{1 \leq j \leq i} \frac{h_{j}}{\left(1-h_{j}\right) n_{j}{ }^{\prime}}
$$

Essa aproximação estabelecida por Greenwood em 1926 permite-nos obter intervalos de confiança aproximados para a função de sobrevivência.

\subsection{2. $O$ estimador de Kaplan-Meier}

Suponha que tenham sido observados $q$ tempos de morte entre os $n$ indivíduos analisados com $q \leq n$, e considere esses tempos ordenados $t_{l}<t_{2}<\ldots<t_{q}$.

O estimador produto-limite, ou de Kaplan-Meier, pode ser entendido como o caso limite do estimador da tábua de vida padrão quando o número de partições no intervalo $[0, \Im]$ tende a infinito $(k \rightarrow \infty)$. Assim sendo, os intervalos tendem a conter no máximo um tempo de morte e, mais ainda, os seus extremos irão convergir para os valores $t_{1}<t_{2}<\ldots<t_{q}$. Podem ocorrer observações empatadas, ou seja, mais de uma falha no tempo $t_{i}$, contudo, em caso de empate entre censura e morte, assume-se que a última ocorre antes e a primeira não contribui naquele instante. Portanto, considerando agora que $n_{i}$ seja o número de indivíduos em risco no instante $t_{i}$ e $s_{i}$ o número de mortes no instante $t_{i}$, o estimador da taxa de risco discreta em $t_{i}$ é dado por

$$
\tilde{h}_{i}= \begin{cases}\frac{l}{n_{i}} & \text { sem observações empatadas } ; \\ \frac{s_{i}}{n_{i}} & \text { na presença de observações empatadas. }\end{cases}
$$


Consequentemente, o estimador de Kaplan-Meier para a função de sobrevivência em um instante $t$ qualquer, no caso em que existem observações empatadas, reduz-se a

$$
\widetilde{G}(t)=\prod_{i: t_{i}<t}\left(\frac{n_{i}-s_{i}}{n_{i}}\right) .
$$

\subsubsection{Propriedades do estimador de Kaplan-Meier}

O estimador de Kaplan-Meier para a função de sobrevivência coincide com o estimador de máxima verossimilhança e possui várias propriedades assintóticas sendo a principal delas o fato de, para $k \rightarrow \infty$ e $n \rightarrow \infty, \widetilde{G}(t)$ ser um estimador consistente para $G(t)$, sob a condição de que os tempos de vida e censura sejam independentes. Mais ainda, a distribuição assintótica de $\sqrt{n}(\widetilde{G}(t)-G(t))$ é Normal com média zero e variância assintótica estimada pela fórmula de Greenwood. Uma prova formal da consistência desse estimador e da sua distribuição assintótica pode ser vista em Lima (1988).

Miller (1983) discute a eficiência desse estimador com relação ao estimador de máxima verossimilhança de duas distribuições para o tempo de sobrevivência: exponencial e Weibull; tal eficiência foi calculada para várias frações de sobrevivência e proporções de censura. Através desse estudo o autor mostra que a eficiência assintótica do estimador de Kaplan-Meier é baixa, principalmente para amostras com alta proporção de censura ou para frações de sobrevivência extremas, ou seja, próximas de zero ou de um. Para frações de sobrevivência altas, referentes aos períodos mais afastados do início do estudo, modelos paramétricos devem ser considerados como uma alternativa para aumentar a precisão das estimativas. 


\subsection{Comparação de duas curvas de sobrevivência}

O interesse em comparar funções de sobrevivência de diferentes grupos é bastante comum, principalmente em pesquisas biomédicas. As ferramentas gráficas são largamente utilizadas e são úteis para visualizar o comportamento dos grupos, além disso, existem vários testes para dados com e sem censura. Para efeito de ilustração será apresentada a técnica de Mantel-Haenszel (1966) para comparar duas curvas de sobrevivência, também denominada teste de "Log-Rank".

Seja $n_{i j}$ o número de indivíduos em risco no instante $t_{i}$ para o $j$-ésimo grupo e $s_{i j}$ o número de mortes no instante $t_{i}$ para o $j$-ésimo grupo. Para cada um dos tempos de falha observados, os dados podem ser representados através de uma tabela de contingência $2 \times 2$, exemplificada pela Tabela 1.1 .

Tabela 1.1: Tabela de contingência usada no cálculo da estatística de Mantel-Haenszel.

\begin{tabular}{cccc}
\hline Grupo & $\begin{array}{c}\text { número de } \\
\text { falhas }\end{array}$ & $\begin{array}{c}\text { número de } \\
\text { sobreviventes }\end{array}$ & Total \\
\hline 1 & $s_{i l}$ & $n_{i l}-s_{i l}$ & $n_{i l}$ \\
2 & $s_{i 2}$ & $n_{i 2}-s_{i 2}$ & $n_{i 2}$ \\
\hline Total & $s_{i}$ & $n_{i}-s_{i}$ & $n_{i}$ \\
\hline
\end{tabular}

Suponha que $s_{i j} \sim \operatorname{bin}\left(n_{i j}, \pi_{i j}\right)$, onde $\pi_{i j}$ denota a probabilidade de morte no instante $t_{i}$ para o $j$-ésimo grupo. A razão de chances de morte entre os grupos $1 \mathrm{e} 2$, no instante $t_{i}$, é definida por

$$
\Psi_{i}=\frac{\pi_{i 1} /\left(1-\pi_{i 1}\right)}{\pi_{i 2} /\left(1-\pi_{i 2}\right)}=\frac{\pi_{i 1}\left(1-\pi_{i 2}\right)}{\pi_{i 2}\left(1-\pi_{i 1}\right)} .
$$


Chances de morte iguais nos dois grupos, no instante $t_{i}$, implicam em $\Psi_{i}=1$. Essa hipótese é usualmente testada através da distribuição hipergeométrica central, cuja função de probabilidades é dada por

$$
f\left(s_{i 1} \mid s_{i}, \Psi_{i}=1\right)=\frac{\left(\begin{array}{c}
n_{i 1} \\
s_{i 1}
\end{array}\right)\left(\begin{array}{c}
n_{i 2} \\
s_{i 2}
\end{array}\right)}{\left(\begin{array}{c}
n_{i 1}+n_{i 2} \\
s_{i}
\end{array}\right)}
$$

com média $E\left(s_{i l} \mid s_{i}, \Psi_{i}=1\right)=\frac{n_{i l} s_{i}}{n_{i}}$ e variância $\operatorname{Var}\left(s_{i l} \mid s_{i}, \Psi_{i}=1\right)=\frac{n_{i l} n_{i 2} s_{i}\left(n_{i}-s_{i}\right)}{n_{i}^{2}\left(n_{i}-1\right)}$. Nesse contexto, comparar duas curvas de sobrevivência equivale a testar a inexistência de associação entre grupo e morte em todos os tempos de falhas observalos, ou seja,

$$
\mathrm{H}_{0}: \Psi_{l}=\Psi_{2}=\ldots=\Psi_{k}=I
$$

Para tanto, Mantel e Haenszel (1966) propuseram um teste cuja estatística é dada por

$$
\begin{aligned}
& X^{2}= \frac{\left(\sum_{i=1}^{k} s_{i 1}-\sum_{i=1}^{k} E\left(s_{i 1} \mid s_{i}, \Psi_{i}=I\right)\right)^{2}}{\sum_{i=1}^{k} \operatorname{Var}\left(s_{i 1} \mid s_{i}, \Psi_{i}=I\right)} \\
&=\left(\sum_{i=1}^{k}\left(s_{i 1}-n_{i 1} s_{i} / n_{i}\right)\right)^{2} \\
& \sum_{i=1}^{k} n_{i I} n_{i 2} s_{i}\left(n_{i}-s_{i}\right) / n_{i}^{2}\left(n_{i}-I\right)
\end{aligned}
$$

Essa estatística possui distribuição nula assintótica qui-quadrado com 1 grau de liberdade, isto é $P\left\{X^{2} \geq c\right\} \cong P\left\{\chi_{1}^{2} \geq c\right\}$, para $c \geq 0$. 
O teste de Log-Rank pode ser generalizado para comparar funções de sobrevivência de três ou mais grupos (ver Lee, 1980).

\subsection{Exemplos}

Considere um exemplo hipotético com a finalidade de exibir os cálculos referentes ao estimador de Kaplan-Meier e à estatística de Mantel-Haenszel.

Exemplo 1.1: 16 pacientes com uma certa doença foram acompanhados e divididos em dois grupos aos quais foram aplicados tratamentos diferentes. Observou-se o tempo até a morte desses indivíduos e obteve-se os resultados a seguir:

Tempo de vida (em meses) para 8 pacientes em cada grupo:

Grupo 1: 3, 5, 7, 9*, 13, 22, 25, 35*;

Grupo 2: 6, 7, 8, 12*, 20*, 23, 33, 34 .

Obs.: Os asteriscos indicam pacientes cujo tempo de vida foi censurado.

O cálculo do estimador de Kaplan-Meier e o intervalo com $95 \%$ de confiança para a função de sobrevivência estão apresentados na Tabela 1.2.

Tabela 1.2: Estimativas de Kaplan-Meier para os dois grupos estudados.

\begin{tabular}{|c|c|c|c|c|c|c|c|c|c|}
\hline \multicolumn{5}{|c|}{ Grupo 1} & \multicolumn{5}{|c|}{ Grupo 2} \\
\hline$t_{i}$ & $n_{i}$ & $s_{i}$ & $\widetilde{G}\left(t_{i}\right)$ & I.C. $\operatorname{para} G\left(t_{i}\right)$ & $t_{i}$ & $n_{i}$ & $s_{i}$ & $\widetilde{G}\left(t_{i}\right)$ & I.C. $\operatorname{para} G\left(t_{i}\right)$ \\
\hline 3 & 8 & 1 & 0,87 & {$[0,67 ; 1,00]$} & 2 & 8 & 1 & 0,87 & {$[0,67 ; 1,00]$} \\
\hline 5 & 7 & 1 & 0,75 & {$[0,50 ; 1,00]$} & 6 & 7 & 1 & 0,75 & {$[0,50 ; 1,00]$} \\
\hline 7 & 6 & 1 & 0,62 & {$[0,37 ; 1,00]$} & 10 & 6 & 1 & 0,62 & {$[0,37 ; 1,00]$} \\
\hline 13 & 4 & 1 & 0,47 & {$[0,21 ; 1,00]$} & 23 & 3 & 1 & 0,42 & {$[0,16 ; 1,00]$} \\
\hline 22 & 3 & 1 & 0,31 & {$[0,10 ; 0,95]$} & 33 & 2 & 1 & 0,21 & {$[0,04 ; 1,00]$} \\
\hline 25 & 2 & 1 & 0,16 & {$[0,03 ; 0,93]$} & 34 & 1 & 1 & 0 & - \\
\hline
\end{tabular}


A Figura 1.2 ilustra as curvas de sobrevivência dos dois grupos submetidos aos dois diferentes tratamentos. Através desse gráfico é possível observar que não parece haver um tratamento melhor, em outras palavras, o gráfico indica que os tratamentos produzem o mesmo efeito na proporção de sobreviventes. Para verificar se existe diferença significativa entre os tratamentos aplicou-se o teste de Mantel-Haenszel. Os cálculos estão apresentados na Tabela 1.3.

Tabela 1.3: Cálculo da estatística de Mantel-Haenszel.

\begin{tabular}{cccccccc}
\hline$t_{i}$ & $n_{i}$ & $n_{i 1}$ & $s_{i}$ & $s_{i 1}$ & $E\left(s_{i 1}\right)$ & $s_{i 1}-E\left(s_{i 1}\right)$ & $\operatorname{Var}\left(s_{i 1}\right)$ \\
\hline 2 & 16 & 8 & 1 & 0 & 0,50 & $-0,50$ & 0,25 \\
3 & 15 & 8 & 1 & 1 & 0,53 & 0,47 & 0,25 \\
5 & 14 & 7 & 1 & 1 & 0,50 & 0,50 & 0,25 \\
6 & 13 & 6 & 1 & 0 & 0,46 & $-0,46$ & 0,25 \\
7 & 12 & 6 & 1 & 1 & 0,50 & 0,50 & 0,25 \\
10 & 10 & 4 & 1 & 0 & 0,40 & $-0,40$ & 0,24 \\
13 & 8 & 4 & 1 & 1 & 0,50 & 0,50 & 0,25 \\
22 & 6 & 3 & 1 & 1 & 0,50 & 0,50 & 0,25 \\
23 & 5 & 2 & 1 & 0 & 0,40 & $-0,40$ & 0,24 \\
25 & 4 & 2 & 1 & 1 & 0,50 & 0,50 & 0,25 \\
33 & 3 & 1 & 1 & 0 & 0,33 & $-0,33$ & 0,22 \\
34 & 2 & 1 & 1 & 0 & 0,50 & $-0,50$ & 0,25 \\
\hline
\end{tabular}

Obtendo-se

$$
X^{2}=\frac{0,37^{2}}{2,95}=0,05 \text {, }
$$

cujo nível descritivo $(p=0,829)$ indica que não há diferença significativa entre os dois tratamentos, ou seja, eles parecem produzir o mesmo efeito na proporção de sobreviventes. 


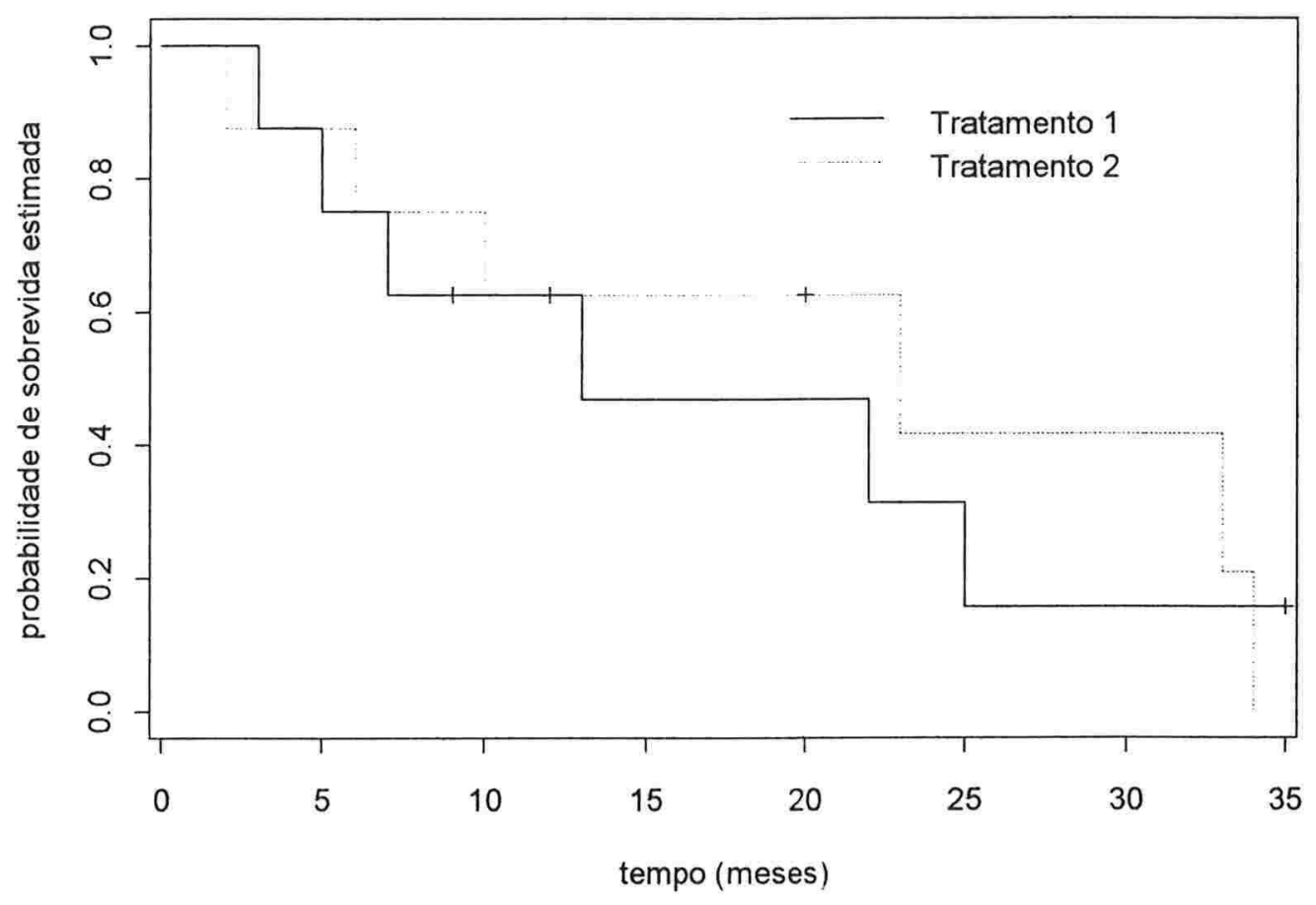

Figura 1.2: Estimativas da probabilidade de sobrevivência obtidas através do método de Kaplan-Meier para os tratamentos 1 e 2.

O exemplo apresentado a seguir foi descrito por Efron (1988) e será usado para ilustrar a metodologia estudada no capítulo seguinte.

Exemplo 1.2: Um estudo de seguimento foi realizado com 96 pacientes com câncer na cabeça e pescoço. Esses pacientes foram divididos em dois grupos aos quais foram aplicados tratamentos diferentes: radioterapia (Tratamento A) e quimioterapia associada a radioterapia (Tratamento B). O grupo submetido ao tratamento A foi observado por 47 meses, enquanto que o outro grupo foi observado por 77 meses. 
Suponha que os períodos observados para os dois grupos tenham sido divididos em intervalos que podem ser de um mês, dois meses ou metade de um mês. Os resultados obtidos nos dois grupos estão apresentados no Apêndice A. Note que esse conjunto de dados possui observações censuradas. Como os pacientes não entraram no estudo necessariamente na mesma data, esse tipo de censura é denominada censura aleatória.

Construindo a curva de Kaplan-Meier para os dois grupos e aplicando o teste de Mantel-Haenszel conclui-se que o tratamento B é significativamente melhor que o A $(p=0,022)$, a um nível de significância de 0,05 , como pode ser visualizado na Figura 1.3.

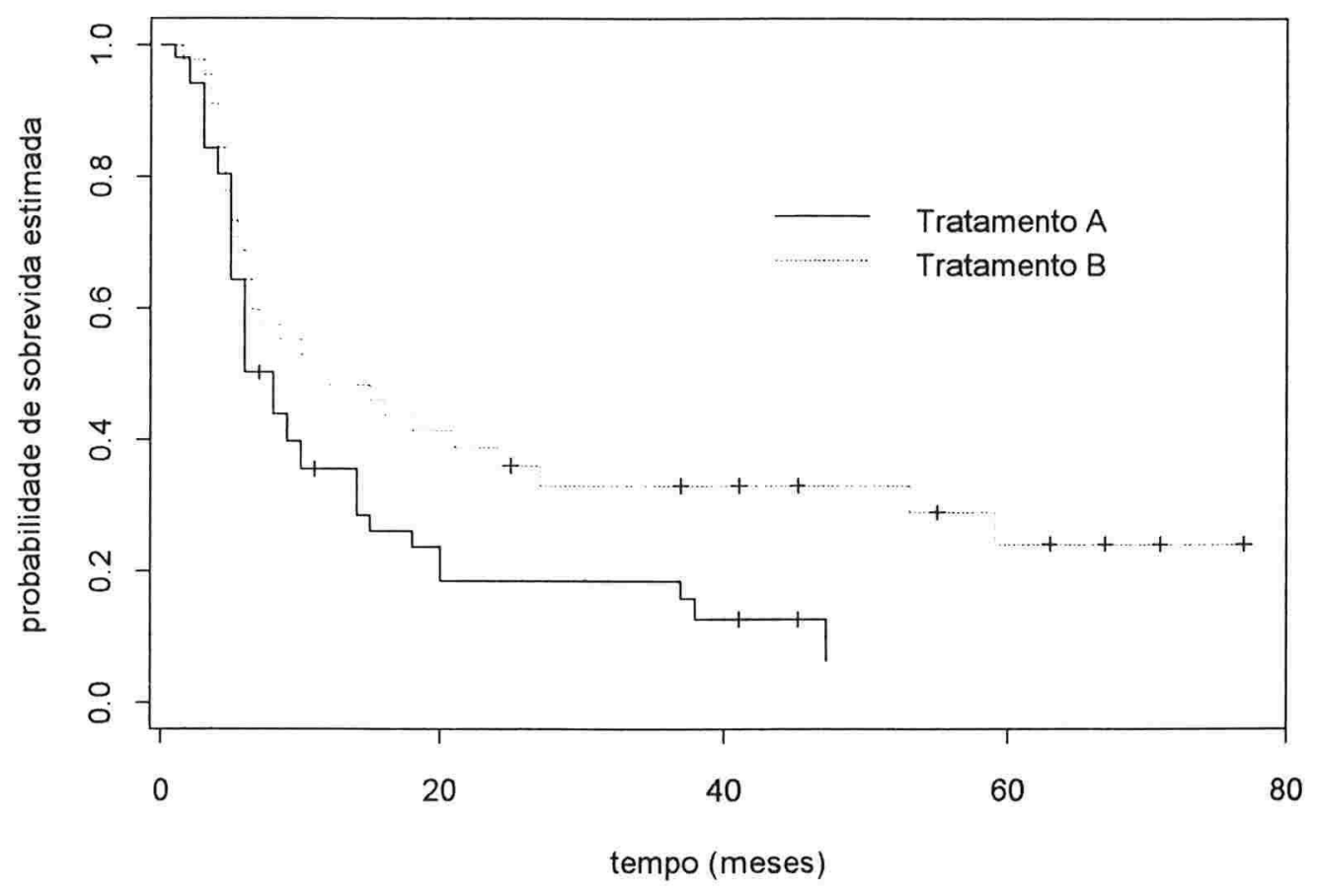

Figura 1.3: Estimativas da probabilidade de sobrevivência obtidas através do método de Kaplan-Meier para os tratamentos A e B. 
Durante muitos anos os estudos referentes a dados de sobrevivência tiveram como principal interesse estimar a função de sobrevivência e comparar distribuições de vários grupos. Atualmente o estudo acerca da função de risco também tem sido de relevante importância. No Capítulo 2 será abordada uma técnica alternativa para analisar dados de sobrevivência, usando regressão logística e "splines" para modelar a taxa de risco. Trata-se de uma estimação paramétrica para problemas em que se tem interesse em estudar o comportamento da taxa de risco ao longo do tempo. Além disso, é possível também obter estimativas para a função de sobrevivência e seu desvio-padrão assintótico, consistindo portanto, uma alternativa à estimação não-paramétrica. 


\section{CAPÍTULO 2}

\section{MODELO DE REGRESSÃO LOGÍSTICA PARCIAL}

Neste capítulo será apresentada uma técnica paramétrica alternativa à de Kaplan-Meier para a estimação da taxa de risco e da curva de sobrevivência para dados censurados. Tal técnica, denominada regressão logística parcial, é uma aplicação direta da regressão logística e a idéia básica não difere muito do estimador de Kaplan-Meier. De fato, quando o número de parâmetros do modelo paramétrico aumenta, as estimativas obtidas aproximam-se das estimativas de Kaplan-Meier.

Como já foi mencionado anteriormente, em regressão paramétrica, é assumida uma distribuição para o tempo de sobrevivência fixando a forma da função de risco. $\mathrm{Na}$ metodologia a ser apresentada, procura-se o melhor ajuste para a função de risco sem a 
preocupação de conhecer qual a distribuição da função de sobrevivência, apesar de, em alguns casos, ela ser facilmente determinada.

Através da regressão logística parcial pode-se obter estimativas para a taxa de risco e função de sobrevivência, bem como para seus respectivos desvios-padrão. A metodologia em questão será apresentada através do Exemplo 1.2 descrito no Capítulo 1.

\subsection{Regressão logística parcial}

Para definir o modelo em questão será utilizada a notação já apresentada no Capítulo 1 considerando-se a discretização indicada na Figura 1.1. A suposição básica necessária desse modelo é que, para $n_{i}$ fixado, o número de mortes em cada intervalo $I_{i}(i=1, \ldots, k)$ ocorra de maneira independente segundo uma distribuição binomial, ou seja,

$$
s_{i} \mid n_{i} \sim \operatorname{bin}\left(n_{i}, h_{i}\right) \text { independentes, para } i=1, \ldots, k \text {, }
$$

onde $h_{i}$ é a taxa de risco discreta ou probabilidade condicional de morte no $i$-ésimo intervalo, como definido anteriormente. Em outras palavras, $s_{i} \mid n_{i}$ tem função de probabilidades

$$
\left(\begin{array}{c}
n_{i} \\
s_{i}
\end{array}\right) h_{i} s_{i}\left(1-h_{i}\right)^{n_{i}-s_{i}}
$$

O objetivo do modelo em estudo é estimar a taxa de risco discreta $h_{i}$ através da técnica de regressão logística tomando o tempo como variável explicativa, tal que

$$
\log \left(\frac{h_{i}}{I-h_{i}}\right)=\sum_{j=1}^{p} x_{i j} \alpha_{j}=x_{i}^{T} \alpha
$$


onde $x_{i}=\left(x_{i 1}, \ldots, x_{i p}\right)^{T}$ contém $p$ funções do tempo e $\alpha=\left(\alpha_{1}, \ldots, \alpha_{p}\right)^{T}$ é um vetor com $p$ parâmetros. Para ilustrar, considere uma regressão logística quadrática no tempo, isto é, o logit da taxa de risco discreta variando segundo uma função quadrática do tempo,

$$
\log i t\left(h_{i}\right)=\boldsymbol{x}_{i}^{T} \alpha=\left(1, t_{i}, t_{i}^{2}\right)\left(\begin{array}{l}
\alpha_{1} \\
\alpha_{2} \\
\alpha_{3}
\end{array}\right)=\alpha_{1}+\alpha_{2} t_{i}+\alpha_{3} t_{i}^{2} .
$$

Efron sugere utilizar $t_{i}$ o ponto médio do $i$-ésimo intervalo para representá-lo e isso implica em fazer a suposição de que os eventos ocorrem de maneira uniforme ao longo do intervalo.

Dessa forma, é fácil verificar que a taxa de risco discreta pode ser expressa por

$$
h_{i}=\left\{1+e^{-\alpha_{1}-\alpha_{2} t_{i}-\alpha_{3} t_{i}^{2}}\right\}^{-1}, \text { para } i=1, \ldots, k
$$

Através da regressão logística, assumindo $s_{i} \mid n_{i} \sim \operatorname{bin}\left(n_{i}, h_{i}\right)$ independentes, será obtido o estimador de máxima verossimilhança $\hat{\alpha}$, utilizando métodos iterativos, para em seguida serem estimados a taxa de risco, a função de sobrevivência e seus respectivos desvios-padrão.

É importante ressaltar que, como a distribuição binomial pertence à família exponencial (McCullagh e Nelder, 1989), a regressão logística nada mais é do que um modelo linear generalizado cuja função de ligação entre a média e o preditor linear é a logit. Portanto, toda teoria referente à essa classe de modelos pode ser aplicada para o modelo em estudo.

\subsubsection{Estimação da taxa de risco discreta e função de sobrevivência}

Considere $\mu_{i}$ como sendo a média do número de mortes no $i$-ésimo intervalo. $\mathrm{O}$ logaritmo da função de verossimilhança do modelo em estudo é dado por 


$$
\begin{aligned}
L=L(\mu, s) & =\sum_{i=1}^{k} L\left(\mu_{i}, s_{i}\right)=\sum_{i=1}^{k} \log \left\{\left(\begin{array}{c}
n_{i} \\
s_{i}
\end{array}\right) h_{i} s_{i}\left(1-h_{i}\right)^{n_{i}-s_{i}}\right\} \\
& =\sum_{i=1}^{k}\left(\log \left(\begin{array}{c}
n_{i} \\
s_{i}
\end{array}\right)+s_{i} \log \left(\frac{h_{i}}{1-h_{i}}\right)+n_{i} \log \left(1-h_{i}\right)\right) .
\end{aligned}
$$

Para derivar o estimador de máxima verossimilhança $\hat{\alpha}$ e estudar sua distribuição assintótica, deve-se obter o vetor escore e a matriz de informação de Fisher, dadas, respectivamente, por

$$
U(\alpha)=\left(\frac{\partial}{\partial \alpha_{1}}, \ldots, \frac{\partial L}{\partial \alpha_{p}}\right)^{T}=X^{T}(s-n h)
$$

$\mathrm{e}$

$$
I(\alpha)=E\left[-\frac{\partial^{2} L}{\partial \alpha \partial \alpha^{T}}\right]=E\left(\begin{array}{ccc}
-\frac{\partial^{2} L}{\partial \alpha_{1}^{2}} & \cdots & -\frac{\partial^{2} L}{\partial \alpha_{1} \partial \alpha_{p}} \\
\vdots & \ddots & \vdots \\
-\frac{\partial^{2} L}{\partial \alpha_{p} \partial \alpha_{1}} & \cdots & -\frac{\partial^{2} L}{\partial \alpha_{p}^{2}}
\end{array}\right)=\boldsymbol{X}^{T} \operatorname{diag}\left(n_{i} V_{i}\right) \boldsymbol{X}=\boldsymbol{X}^{T} \boldsymbol{V} \boldsymbol{X}
$$

onde $\boldsymbol{X}_{(p x k)}^{T}=\left(x_{1}, x_{2}, \cdots, x_{k}\right), \boldsymbol{s}_{(k \times 1)}=\left[s_{1}, s_{2}, \cdots, s_{k}\right]^{T}, \boldsymbol{n} \boldsymbol{h}_{(k \times 1)}=\left[n_{1} h_{1}, n_{2} h_{2}, \cdots, n_{k} h_{k}\right]^{T} \mathrm{e}$ $V_{(k \times k)}=\operatorname{diag}\left[n_{1} V_{l}, n_{2} V_{2}, \cdots, n_{k} V_{k}\right]$ denota a matriz de pesos, com $V_{i}=h_{i}\left(1-h_{i}\right)$.

O estimador de máxima verossimilhança $\hat{\alpha}$ é obtido resolvendo-se o sistema de equações $U(\alpha)=0$ e estimativas para esse vetor de parâmetros são fornecidas por processos iterativos. O processo iterativo para a obtenção de estimativas de máxima verossimilhança de $\alpha$ mais utilizado é o de Newton-Raphson, definido pela expansão da função escore $U(\alpha)$ em torno de um valor inicial $\alpha^{(0)}$ tal que 


$$
U(\alpha) \cong U\left(\alpha^{(0)}\right)+U^{\prime}\left(\alpha^{(0)}\right)\left(\alpha-\alpha^{(0)}\right)
$$

onde $U^{\prime}(\alpha)$ denota a primeira derivada de $U(\alpha)$ com relação a $\alpha$. Assim, repetindo-se o procedimento acima, chega-se ao processo iterativo

$$
\alpha^{(m+l)}=\alpha^{(m)}+\left\{-U^{\prime}\left(\alpha^{(m)}\right)\right\}^{-1} U\left(\alpha^{(m)}\right), \text { para } \mathrm{m}=0,1,2,3, \ldots
$$

A solução do processo iterativo na convergência é dada por

$$
\hat{\alpha}=\left(X^{T} \hat{V} X\right)^{-1} X^{T} \hat{V} z
$$

onde

$$
z=X \hat{\alpha}+\hat{V}^{-1}(s-n \hat{h})
$$

Note que (2.3) coincide com a solução de mínimos quadrados de uma regressão linear, ponderada por $\hat{V}$, onde a variável resposta é $Z$ e a matriz modelo é $X$.

A grande vantagem de utilizar o estimador de máxima verossimilhança (e.m.v.) consiste no fato de o mesmo possuir propriedades bastante importantes a partir das quais pode-se estudar a sua distribuição assintótica e obter estimadores para funções de $\alpha$. Aqui estão descritas três propriedades importantes do estimador de máxima verossimilhança.

Propriedade 1. Se $\hat{\alpha}$ é um e.m.v. de $\alpha$, então $\mathrm{f}(\hat{\alpha})$ é e.m.v. de $\mathrm{f}(\alpha)$ (onde $\mathrm{f}($.) é uma função injetora). Essa é conhecida como propriedade da invariância do estimador de máxima verossimilhança. 
Propriedade 2. Se $\hat{\alpha}$ é um e.m.v. de $\alpha$, sob certas condições de regularidade, a distribuição assintótica de $\hat{\alpha}$ é dada por

$$
\hat{\alpha} \sim N\left(\alpha, I^{-1}(\alpha)\right)
$$

Propriedade 3. Se $\hat{\alpha}$ é o e.m.v. de $\alpha$, então possui a menor variância assintótica entre todos os estimadores assintóticamente não-viesados e é dito eficiente (ver DeGroot, 1989).

Portanto, pela propriedade 2 a matriz de variância-covariância assintótica de $\hat{\alpha}$ é a inversa da matriz de informação de Fisher $\left(\operatorname{Cov}(\hat{\alpha})=I^{-1}(\alpha)\right)$ já determinada anteriormente. Além disso, pela propriedade da invariância, os estimadores de máxima verossimilhança da taxa de risco discreta e, consequentemente, da função de sobrevivência, são respectivamente, expressos por

$$
\hat{h}_{i}=\frac{1}{1+e^{-x_{i}^{T} \hat{\alpha}}}
$$

$\mathrm{e}$

$$
\hat{G}_{i}=\prod_{j=l}^{i}\left(I-\hat{h}_{j}\right) .
$$

\subsubsection{Estimação dos desvios-padrão da taxa de risco e função de sobrevivência}

Para determinar os desvios-padrão de $\hat{h}_{\imath}$ e $\hat{G}_{i}$, deve-se utilizar o método Delta, descrito a seguir. Se o vetor $\hat{\alpha}$ tem distribuição $N\left(\alpha, I^{-1}(\alpha)\right)$ então, assintoticamente, o vetor $f(\hat{\alpha})=\left[f_{l}(\hat{\alpha}), \ldots, f_{k}(\hat{\alpha})\right]$ tem distribuição

$$
N\left(f(\alpha) ;\left\{f^{\prime}(\alpha)\right\} I^{-1}(\alpha)\left\{f^{\prime}(\alpha)\right\}^{T}\right)
$$


Assim, considerando novamente que a taxa de risco discreta é uma função injetora de $\alpha$ podemos determinar a matriz de variância-covariância correspondente. Tem-se que,

$$
h_{i}=f_{i}(\alpha)=\frac{1}{1+e^{-x_{i}^{T} \alpha}}
$$

e daí segue que

$$
f_{i}^{\prime}(\alpha)=\left(\frac{-x_{i l} e^{-x_{i}^{T} \alpha}}{\left(1+e^{-x_{i}^{T} \alpha}\right)^{2}}, \ldots, \frac{-x_{i p} e^{-x_{i}^{T} \alpha}}{\left(1+e^{-x_{i}^{T} \alpha}\right)^{2}}\right)=h_{i}\left(1-h_{i}\right)\left(-x_{i l}, \ldots,-x_{i p}\right)=-h_{i}\left(1-h_{i}\right) x_{i}^{T}
$$

onde, como já foi dito, $x_{i}^{T}=\left(x_{i 1}, \ldots, x_{i p}\right)$. Logo, a variância e o desvio-padrão de $\hat{h}_{i}$ são dados, respectivamente, por

$$
\begin{aligned}
\operatorname{Var}\left(\hat{h}_{i}\right) & =h_{i}\left(1-h_{i}\right) x_{i}^{T} I^{-1}(\alpha) h_{i}\left(1-h_{i}\right) x_{i} \\
& =\left(h_{i}\left(I-h_{i}\right)\right)^{2} x_{i}^{T} I^{-1}(\alpha) x_{i}
\end{aligned}
$$

e

$$
d p\left(\hat{h}_{i}\right)=h_{i}\left(I-h_{i}\right)\left(\boldsymbol{x}_{i}^{T} I^{-1}(\alpha) x_{i}\right)^{1 / 2}=V_{i}\left(x_{i}^{T} I^{-1}(\alpha) x_{i}\right)^{1 / 2}
$$

Da mesma forma, a função de sobrevivência também pode ser escrita como uma função do vetor de parâmetros $\alpha$, 


$$
G_{i}=f_{i}(\alpha)=\prod_{j=1}^{i}\left(1-h_{j}\right)=e^{\sum_{j=1}^{l} \log \left(\frac{e^{-\boldsymbol{x}_{j}^{T} \boldsymbol{\alpha}}}{1+e^{-\boldsymbol{x}_{j}^{T} \alpha}}\right)},
$$

de onde obtém-se

$$
\begin{aligned}
\frac{\partial f_{i}(\alpha)}{\partial \alpha_{m}} & =G_{i} \sum_{j=1}^{i} \frac{I+e^{-\boldsymbol{x}_{j}^{T} \alpha}}{e^{-\boldsymbol{x}_{j}^{T} \alpha}}\left(\frac{\left(1+e^{-\boldsymbol{x}_{j}^{T} \alpha}\right)\left(-x_{j m}\right) e^{-\boldsymbol{x}_{j}^{T} \alpha}-e^{-\boldsymbol{x}_{j}^{T} \alpha}\left(-x_{j m}\right) e^{-\boldsymbol{x}_{j}^{T} \alpha}}{\left(1+e^{-\boldsymbol{x}_{j}^{T} \alpha}\right)}\right) \\
& =G_{i} \sum_{j=1}^{i} \frac{1}{I-h_{j}}\left(-x_{j m} h_{j}\left(1-h_{j}\right)\right)=G_{i} \sum_{j=1}^{i}-x_{j m} h_{j}=-G_{i} \sum_{j=1}^{i} x_{j m} h_{j}, \operatorname{para} m=1, \ldots, p
\end{aligned}
$$

Portanto, a variância e o desvio-padrão de $\hat{G}_{i}$ ficam, respectivamente, dados por

$$
\begin{aligned}
\operatorname{Var}\left(\hat{G}_{i}\right) & =G_{i}\left(-\sum_{j=1}^{i} x_{j l} h_{j}, \ldots,-\sum_{j=1}^{i} x_{j p} h_{j}\right) I^{-1}(\alpha) G_{i}\left(\begin{array}{c}
-\sum_{j=1}^{i} x_{j l} h_{j} \\
\vdots \\
-\sum_{j=1}^{i} x_{j p} h_{j}
\end{array}\right) \\
& =G_{i}^{2}\left(\sum_{j=1}^{i} h_{j} x_{j}\right)^{T} I^{-1}(\alpha)\left(\sum_{j=1}^{i} h_{j} x_{j}\right)
\end{aligned}
$$

e

$$
d p\left(\hat{G}_{i}\right)=G_{i}\left\{\left(-\sum_{j=1}^{i} h_{j} x_{j}\right)^{T} I^{-1}(\alpha)\left(-\sum_{j=1}^{i} h_{j} x_{j}\right)\right\}^{1 / 2}
$$


Uma vez determinados os desvios-padrão da taxa de risco discreta e da função de sobrevivência e, consequentemente, as distribuições assintóticas dessas quantidades, pode-se construir intervalos de confiança pontuais a partir dos quais obtém-se bandas de confiança. No caso da taxa de risco os limites inferior e superior de um intervalo com $95 \%$ de confiança para o $i$-ésimo intervalo são obtidos, respectivamente, através das expressões

$$
\hat{h}_{i}-1,96 * \hat{V}_{i}\left(x_{i}^{T} I^{-1}(\hat{\alpha}) x_{i}\right)^{1 / 2}
$$

$\mathrm{e}$

$$
\hat{h}_{i}+1,96 * \hat{V}_{i}\left(\boldsymbol{x}_{i}^{T} I^{-1}(\hat{\alpha}) x_{i}\right)^{1 / 2} .
$$

Analogamente, no caso da função de sobrevivência os limites inferior e superior de um intervalo com $95 \%$ de confiança para o $i$-ésimo intervalo são dados, respectivamente, por

$$
\hat{G}_{i}-1,96 * \hat{G}_{i}\left\{\left(-\sum_{j=1}^{i} \hat{h}_{j} x_{j}\right)^{T} I^{-1}(\hat{\alpha})\left(-\sum_{j=1}^{i} \hat{h}_{j} x_{j}\right)\right\}^{1 / 2}
$$

e

$$
\hat{G}_{i}+1,96 * \hat{G}_{i}\left\{\left(-\sum_{j=1}^{i} \hat{h}_{j} x_{j}\right)^{T} I^{-1}(\hat{\alpha})\left(-\sum_{j=1}^{i} \hat{h}_{j} x_{j}\right)\right\}^{1 / 2} .
$$




\subsection{Função desvio}

Considere o modelo saturado em que a função de verossimilhança é estimada por

$$
L(s, s)=\sum_{i=1}^{k} L\left(s_{i}, s_{i}\right) .
$$

Para avaliar a qualidade do ajuste de um modelo linear generalizado pode-se utilizar a função desvio,

$$
D(\hat{\mu}, s)=2\{L(s, s)-L(\hat{\mu}, s)\},
$$

que é uma medida da distância entre o logaritmo da função de verossimilhança do modelo saturado (com $k$ parâmetros) e do modelo sob investigação (com $p$ parâmetros) avaliado na estimativa de máxima verossimilhança $\hat{\alpha}$. Efetuando-se os cálculos para o caso da distribuição binomial, a expressão da função desvio fica dada por

$$
D(\hat{\mu}, s)= \begin{cases}2 \sum_{i=1}^{k}\left[s_{i} \log \left(\frac{s_{i}}{n_{i} \hat{h}_{i}}\right)+\left(n_{i}-s_{i}\right) \log \left(\frac{n_{i}-s_{i}}{n_{i}\left(1-\hat{h}_{i}\right)}\right)\right] & 0<s_{i}<n_{i} ; \\ -2 n_{i} \log \left(1-\hat{h}_{i}\right) & s_{i}=0 ; \\ -2 n_{i} \log \hat{h}_{i} & s_{i}=n_{i} .\end{cases}
$$

Em geral a distribuição do desvio segue, sob a hipótese de que o modelo adotado seja verdadeiro, uma distribuição qui-quadrado com $(k-p)$ graus de liberdade. Entretanto, esse resultado é questionável quando $n_{i} h_{i}\left(1-h_{i}\right)$ fica limitado, como é o caso dos modelos em que $n_{i}$ decresce a cada intervalo. Nesse caso, Hosmer e Lemeshow $(1980,1989)$ sugerem uma estatística alternativa para avaliar a qualidade do ajuste, baseada na comparação do número 
observado e esperado de eventos, que no caso é a morte, de $g$ grupos formados. O primeiro grupo deverá conter os $n_{1}{ }^{\prime}$ intervalos correspondentes às $n_{1}{ }^{\prime}$ menores probabilidades ajustadas e assim sucessivamente até o último grupo que deverá conter os $n_{g}{ }^{\prime}$ intervalos correspondentes às $n_{g}{ }^{\prime}$ maiores probabilidades ajustadas. A estatística em questão é definida por

$$
\hat{C}=\sum_{i=1}^{g} \frac{\left(O_{i}-n_{i}{ }^{\prime} \bar{\pi}_{i}\right)^{2}}{n_{i}{ }^{\prime} \bar{\pi}_{i}\left(1-\bar{\pi}_{i}\right)},
$$

onde $O_{i}$ é o número observado de eventos no $i$-ésimo grupo formado e $\bar{\pi}_{i}$ é uma média das probabilidades ajustadas do $i$-ésimo grupo formado. Hosmer e Lemeshow verificaram que a distribuição nula assintótica dessa estatística pode ser aproximada por uma qui-quadrado com $(g-2)$ graus de liberdade. Da mesma forma que a função desvio, um valor suficientemente pequeno dessa estatística indica que, para um número menor de parâmetros, obtém-se um ajuste tão bom quanto o ajuste do modelo saturado.

A grande vantagem da função desvio está no aspecto computacional, já que ela é facilmente obtida, entretanto, como essa estatística pode não ser adequada no caso do modelo estudado, foi construído um programa no Splus para calcular a estatística de Hosmer e Lemeshow. Dessa forma, pode-se concluir com mais segurança quanto ao ajuste dos modelos propostos.

Efron sugere ainda, a utilização da estatística do desvio para verificar a qualidade do ajuste de cada modelo, mas baseada também em um agrupamento dos intervalos de tal forma a garantir que o número de indivíduos em risco em cada grupo formado seja aproximadamente o mesmo. Essa estatística deve, então, ser comparada então com uma qui-quadrado com $g-k$ graus de liberdade, onde $g$ é o número de grupos e $k$ o número de parâmetros do modelo. Portanto, pode ser que a estatística Hosmer e Lemeshow, que é semelhante à essa estatística proposta por Efron, deva ser comparada com uma qui-quadrado com graus de liberdade menor do que $g-2$, no caso dos modelos mais parametrizados. 


\subsection{Teste de hipótese acerca do vetor de parâmetros $\alpha$}

Considere a partição para o vetor de parâmetros $\alpha=\left(\alpha_{1}^{T}, \alpha_{2}^{T}\right)^{T}$, onde $\alpha_{1}$ é um vetor $u$-dimensional e, consequentemente, $\alpha_{2}$ um vetor $(p$ - $u)$-dimensional. Analogamente, considere a partição da matriz modelo $X=\left(X_{1}, X_{2}\right)$, onde $X_{1}$ é uma matriz $\left(k_{x} u\right)$ cujas colunas são relativas ao vetor $\alpha_{1}$ e, da mesma forma, $X_{2}$ uma matriz $\left(k_{x}(p-u)\right)$ cujas colunas são relativas ao vetor $\alpha_{2}$. Nesse contexto, pode-se ter interesse em testar a hipótese $H_{0}: \alpha_{1}=0$ contra $H_{l}$ : $\alpha_{1} \neq 0$. Tomando os logaritmos das funções de verossimilhança correspondentes aos modelos sob $H_{0}$ e $H_{l}$, respectivamente $L\left(\hat{\mu}^{0}, s\right)$ e $L(\hat{\mu}, s)$, a estatística da razão de verossimilhança para testar essa hipótese é definida, nesse caso, por

$$
\varepsilon_{R V}=2\left\{L(\hat{\mu}, s)-L\left(\hat{\mu}^{0}, s\right)\right\}=D\left(\hat{\mu}^{0}, s\right)-D(\hat{\mu}, s),
$$

isto é, a diferença entre os desvios dos modelos sob $H_{0}$ e $H_{l}$. No caso de $0<s_{i}<n_{i}$ a expressão da estatística da razão de verossimilhança é dada por

$$
\varepsilon_{R V}=2 \sum_{i=1}^{k}\left[s_{i} \log \left(\frac{\hat{h}_{i}}{\hat{h}_{i}^{0}}\right)+\left(n_{i}-s_{i}\right) \log \left(\frac{\left(1-\hat{h}_{i}\right)}{\left(1-\hat{h}_{i}^{0}\right)}\right)\right] .
$$

De maneira similar, outras duas estatísticas podem ser utilizadas para testar essa hipótese, a estatística de Wald e de escore. A primeira é expressa na forma

$$
\varepsilon_{W}=\left(\hat{\alpha}_{1}-0\right)^{T} \hat{\operatorname{Var}}^{-1}\left(\hat{\alpha}_{1}\right)\left(\hat{\alpha}_{1}-0\right)
$$

e a segunda

$$
\varepsilon_{S R}=U_{l}\left(\hat{\alpha}^{0}\right)^{T} \widetilde{V} r_{0}\left(\hat{\alpha}_{1}\right) U_{l}\left(\hat{\alpha}^{0}\right) .
$$


onde $\hat{\operatorname{Var}}\left(\hat{\alpha}_{1}\right)$ e $\widetilde{\operatorname{Var}}{ }_{0}\left(\hat{\alpha}_{l}\right)$ indicam, respectivamente, que as variâncias assintóticas de $\hat{\alpha}_{t}$ estão sendo estimadas sob $H_{l}$ e $H_{0}$ e $U_{l}(\alpha)=\frac{\partial}{\partial \alpha_{l}}=X_{l}^{T}(s-n h)$. A expressão da variância assintótica de $\hat{\alpha}_{1}$, é dada por

$$
\begin{aligned}
\operatorname{Var}\left(\hat{\alpha}_{l}\right) & =\left(\boldsymbol{X}_{1}^{T} V \boldsymbol{X}_{l}-\boldsymbol{X}_{l}^{T} V \boldsymbol{X}_{2}\left(\boldsymbol{X}_{2}^{T} V \boldsymbol{X}_{2}\right)^{-l} \boldsymbol{X}_{2}^{T} V \boldsymbol{X}_{l}\right)^{-l} \\
& =\left(\boldsymbol{X}_{1}^{T} \boldsymbol{V}^{l / 2}\left(\boldsymbol{I}-\boldsymbol{P}_{2}\right) \boldsymbol{V}^{1 / 2} \boldsymbol{X}_{l}\right)^{-1}
\end{aligned}
$$

onde $\boldsymbol{P}_{2}=\left(\boldsymbol{V}^{l .2} \boldsymbol{X}_{2}\left(\boldsymbol{X}_{2}^{T} V \boldsymbol{X}_{2}\right)^{-1} \boldsymbol{X}_{2}^{T} \boldsymbol{V}^{1 / 2}\right)^{-1}$ é a matriz de projeção ortogonal de vetores do $\mathfrak{R}^{K}$ no subespaço gerado pelas colunas da matriz $V^{1 / 2} \boldsymbol{X}_{2}$. Logo, as estatísticas de Wald e de escore ficam expressas, respectivamente, nas formas

$$
\varepsilon_{W}=\hat{\alpha}_{1}^{T}\left(\boldsymbol{X}_{1}^{T} \hat{\boldsymbol{V}}^{1 / 2}\left(\boldsymbol{I}-\boldsymbol{P}_{2}\right) \hat{\boldsymbol{V}}^{1 / 2} \boldsymbol{X}_{1}\right) \hat{\alpha}_{1}
$$

e

$$
\varepsilon_{S R}=(s-n \widetilde{h})^{T} X_{l}\left(X_{l}^{T} \widetilde{V}_{0}^{l / 2}\left(I-P_{2}\right) \widetilde{V}_{0}^{1 / 2} X_{l}\right)^{-1} X_{l}^{T}(s-n \widetilde{h}) .
$$

Para estimar a estatística de escore, $V$ deve ser substituído por $\widetilde{V}_{0}$, ou seja, deve ser estimado sob $H_{0}$, embora tenha a forma do modelo em $H_{l}$. Por essa razão, a estatística de escore pode ser muito conveniente quando se tem um modelo complicado na hipótese alternativa, pois é estimada inteiramente sob $H_{0}$. Assim, a estimação dos parâmetros sob $H_{l}$ torna-se necessária somente quando $H_{0}$ é rejeitada.

Assintoticamente e sob a hipótese nula, as estatísticas $\varepsilon_{R V}, \varepsilon_{W}$ e $\varepsilon_{S R}$ seguem uma distribuição qui-quadrado $\operatorname{com} u$ graus de liberdade. 
Uma outra maneira de testar a hipótese em questão é através da estatística $F$, definida, assim como a estatística da razão de verossimilhança, a partir dos desvios correspondentes aos modelos sob $H_{0}$ e $H_{l}$,

$$
F=\frac{\left\{D\left(\hat{\mu}^{o}, s\right)-D(\hat{\mu}, s)\right\} / u}{D(\hat{\mu}, s) /(k-p)}
$$

e no caso $0<s_{i}<n_{i}$, a estatística $F$ assume a forma

$$
F=\frac{\sum_{i=1}^{k}\left[s_{i} \log \left(\frac{\hat{h}_{i}}{\hat{h}_{i}^{0}}\right)+\left(n_{i}-s_{i}\right) \log \left(\frac{\left(1-\hat{h}_{i}\right)}{\left(1-\hat{h}_{i}^{0}\right)}\right)\right] / u}{2 \sum_{i=1}^{k}\left[s_{i} \log \left(\frac{s_{i}}{n_{i} \hat{h}_{i}}\right)+\left(n_{i}-s_{i}\right) \log \left(\frac{n_{i}-s_{i}}{n_{i}\left(1-\hat{h}_{i}\right)}\right)\right] /(k-p)} .
$$

A distribuição assintótica sob $H_{0}$ dessa estatística é uma $F_{u,(k-p)}$.

Para ilustrar, considere o modelo (2.2) em que o logit da chance de morte varia segundo uma função quadrática no tempo. Nesse caso, o vetor de parâmetros é dado por $\alpha=\left(\alpha_{1}, \alpha_{2}, \alpha_{3}\right)^{T}$ e pode-se ter interesse em testar se o coeficiente quadrático é significativamente diferente de zero, ou seja, testar as hipóteses

$$
\begin{aligned}
& H_{0}: \alpha_{3}=0 \\
& H_{1}: \alpha_{3} \neq 0 .
\end{aligned}
$$

Deve-se, portanto, considerar a partição do vetor de parâmetros em $\alpha_{3}$ e $\left(\alpha_{1}, \alpha_{2}\right)^{T}$. Da mesma forma a matriz modelo 


$$
\boldsymbol{X}=\left(\begin{array}{ccc}
l & t_{1} & t_{l}^{2} \\
l & t_{2} & t_{2}^{2} \\
\vdots & \vdots & \vdots \\
l & t_{k} & t_{k}^{2}
\end{array}\right)
$$

também deve ser particionada em $X_{1}=\left(\begin{array}{llll}t_{1}^{2} & t_{2}^{2} & \cdots & t_{k}^{2}\end{array}\right)^{T}$ e $X_{2}=\left(\begin{array}{cccc}1 & 1 & \cdots & 1 \\ t_{1} & t_{2} & \cdots & t_{k}\end{array}\right)^{T}$.

Efetuando as contas matriciais obteve-se o seguinte resultado para a variância assintótica do parâmetro $\alpha_{3}$ :

$$
\operatorname{Var}\left(\hat{\alpha}_{3}\right)=v_{4}-\frac{v_{2}^{3}-2 v_{1} v_{2} v_{3}+v_{0} v_{3}^{2}}{v_{0} v_{2}-v_{1}^{2}}
$$

onde $v_{0}=\sum_{i=1}^{k} n_{i} V_{i}, \quad v_{1}=\sum_{i=1}^{k} t_{i} n_{i} V_{i}, \quad v_{2}=\sum_{i=1}^{k} t_{i}^{2} n_{i} V_{i}, \quad v_{3}=\sum_{i=1}^{k} t_{i}^{3} n_{i} V_{i}$ e $v_{4}=\sum_{i=1}^{k} t_{i}^{4} n_{i} V_{i}$

A estatistica de Wald para testar a hipótese de interesse é dada por

$$
\varepsilon_{W}=\frac{\hat{\alpha}_{3}^{2}}{\operatorname{Var}\left(\hat{\alpha}_{3}\right)}
$$

onde $\hat{\operatorname{Var}}\left(\hat{\alpha}_{3}\right)$ é avaliada sob $H_{1}$. Já a estatística de escore toma a forma

$$
\begin{aligned}
\varepsilon_{S R} & =\boldsymbol{X}_{1}^{T}\left(\boldsymbol{s}-\boldsymbol{n} \hat{\boldsymbol{h}}^{0}\right)^{T} \widetilde{\operatorname{Var}}\left(\hat{a}_{3}^{0}\right) \boldsymbol{X}_{l}\left(\boldsymbol{s}-\boldsymbol{n} \hat{\boldsymbol{h}}^{0}\right) \\
& =\left(\sum_{i=1}^{k} t_{i}^{2}\left(s_{i}-n_{i} \hat{h}_{i}^{0}\right)\right)^{2} \widetilde{\operatorname{Var}}\left(\hat{\alpha}_{3}^{0}\right) .
\end{aligned}
$$

onde $\hat{h}_{i}^{0}=\left\{I+e^{-\hat{\alpha}_{1}-\hat{\alpha}_{2} t_{i}}\right\}^{-1}$, para $i=I, \ldots, k$, e a variância $\widetilde{\operatorname{Var}}\left(\hat{\alpha}_{3}^{o}\right)$ é avaliada sob $H_{0}$. 


\subsection{Resíduos e técnicas de diagnóstico}

Como já foi visto, o estimador de máxima verossimilhança de $\alpha$ coincide com a solução de mínimos quadrados de uma regressão linear, ponderada por $\hat{V}$, onde a variável resposta é $Z$, dada por (2.4), e a matriz modelo é $\boldsymbol{X}$. Portanto, o resíduo ordinário dessa regressão é definido pela diferença entre o valor observado e ajustado através do modelo, ponderado pela matriz de pesos

$$
\boldsymbol{r}=\hat{V}^{1 / 2}(z-X \hat{\alpha})=\hat{V}^{-1 / 2}(s-n \hat{\boldsymbol{h}})
$$

Esse resíduo, denominado Studentizado, pode ser escrito, para o $i$-ésimo intervalo, na forma

$$
r_{i}=\frac{\left(s_{i}-n_{i} \hat{h}_{i}\right)}{\sqrt{n_{i} \hat{h}_{i}\left(1-\hat{h}_{i}\right)}}
$$

ou ainda

$$
\boldsymbol{r}=(\boldsymbol{I}-\hat{\boldsymbol{P}}) \hat{\boldsymbol{V}}^{1 / 2} \boldsymbol{z}
$$

onde $\hat{P}=\hat{V}^{1 / 2} X\left(X^{T} \hat{V} X\right)^{-1} X^{T} \hat{V}^{1 / 2}$ desempenha o papel de uma matriz de projeção ortogonal local de $\hat{V}^{1 / 2} z$ nas colunas de $\hat{V}^{1 / 2} \boldsymbol{X}$, possuindo, portanto, as mesmas propriedades da martiz "hat" na regressão normal linear onde $V$ é uma matriz identidade, ou seja, é simétrica e idempotente $\left(\boldsymbol{P}=\boldsymbol{P}^{2}\right)$. Isso assegura que $0 \leq p_{i i} \leq l$ sugerindo a utilização da diagonal principal da matriz $\hat{\boldsymbol{P}}$ para detectar pontos mais afastados no subespaço gerado pelas colunas de $\hat{V}^{1 / 2} X$. No entanto, a ponderação $\hat{V}^{1 / 2}$ não leva necessariamente à mesma interpretação do caso normal linear. Para entender isso, note que $\hat{p}_{i i}$ pode ser escrito na forma

$$
\hat{p}_{i i}=n_{i} \hat{h}_{i}\left(1-\hat{h}_{i}\right) x_{i}^{T}\left(X^{T} \hat{V} X\right)^{-1} x_{i}
$$


e não necessariamente pontos com valores altos para $\boldsymbol{x}_{i}^{T}\left(\boldsymbol{X}^{T} \hat{\boldsymbol{V} X}\right)^{-1} \boldsymbol{x}_{i}$, que seriam no caso normal linear pontos de alto "leverage" (ou alavancas), tem essa propriedade no modelo logístico linear, pois quando a probabilidade ajustada $\hat{h}_{i}$ é muito pequena ou muito grande, $\hat{p}_{i t}$ torna-se, em geral, pequeno o que dificulta a detecção de pontos que estejam afastados no subespaço gerado pelas colunas de $\boldsymbol{X}$. Portanto, $\hat{p}_{i i}$ pode ser interpretado como no caso normal linear somente para $0,1<\hat{h}_{i}<0,9$. Tendência similar pode ser observada para o resíduo Studentizado padronizado

$$
t_{S_{i}}=\frac{r_{i}}{\sqrt{1-\hat{p}_{i i}}},
$$

e para a medida de influência das observações nas estimativas dos coeficientes, conhecida como distância de Cook

$$
L D_{i}=\frac{\hat{p}_{i i}}{\left(1-\hat{p}_{i i}\right)^{2}} \frac{\left(s_{i}-n_{i} \hat{h}_{i}\right)^{2}}{n_{i} \hat{h}_{i}\left(1-\hat{h}_{i}\right)} .
$$

Essa última medida é baseada na comparação dos modelos com e sem a $i$-ésima observação e permite avaliar o impacto da retirada da $i$-ésima observação na verossimilhança do modelo completo (Collet,1991). Espera-se que todos os pontos exerçam a mesma influência nas estimativas dos parâmetros, entretanto, quando um ponto apresenta essa medida muito maior que os demais, ele é usualmente considerado um possível ponto influente e, nesse caso, deve-se investigar a causa.

Outro tipo de resíduo bastante utilizado, definido a partir dos componentes da função desvio

$$
d_{i}=\operatorname{sinal}\left(s_{i}-n_{i} \hat{h}_{i}\right) 2\left[s_{i} \log \left(\frac{s_{i}}{n_{i} \hat{h}_{i}}\right)+\left(n_{i}-s_{i}\right) \log \left(\frac{n_{i}-s_{i}}{n_{i}\left(1-\hat{h}_{i}\right)}\right)\right]^{1 / 2},
$$

é o chamado componente do desvio padronizado, 


$$
t_{D_{i}}=\frac{d_{i}}{\sqrt{\left(1-\hat{p}_{i i}\right)}}
$$

Williams (1984) verificou através de simulações que a distribuição do $t_{D_{i}}$ tende a estar mais próxima da normalidade do que a distribuição de outros resíduos.

Note que como a medida $\hat{p}_{i i}$ depende das probabilidades ajustadas, os resíduos $t_{s_{i}} \mathrm{e}$ $t_{D_{i}}$ e a medida de influência $L D_{i}$ também dependem. A sugestão, portanto, é construir gráficos de $\hat{p}_{i i}, t_{S_{i}}, t_{D_{i}}$ e $L D_{i}$ contra os valores ajustados, que podem ser mais informativos a respeito do posicionamento dos pontos aberrantes e influentes. Espera-se observar as tendências apresentadas na Tabela 2.1 .

Tabela 2.1: Possíveis tendências para algumas medidas de diagnóstico segundo as probabilidades ajustadas.

\begin{tabular}{cccccc}
\hline & \multicolumn{5}{c}{ Probabilidades ajustadas } \\
\cline { 2 - 6 } Medida & $0,0-0,1$ & $0,1-0,3$ & $0,3-0,7$ & $0,7-0,9$ & $0,9-1,0$ \\
\hline$t_{D_{i}}$ & $\begin{array}{c}\text { grande ou } \\
\text { pequeno }\end{array}$ & moderado & moderado & moderado & grande ou \\
& ou pequeno & & pequeno \\
$L D_{i}$ & pequeno & grande & moderado & grande & pequeno \\
$\hat{p}_{i i}$ & pequeno & grande & moderado & grande & pequeno \\
& & & ou pequeno & \\
\hline
\end{tabular}

Desde que a distribuição do resíduo $t_{D_{t}}$ pode ser aproximada pela distribuição normal padrão, outro gráfico que pode ser utilizado para verificar a adequação do modelo, caso ele seja verdadeiro, é o gráfico normal de probabilidades. A idéia por trás desse gráfico é que se os resíduos ordenados são plotados contra uma aproximação dos seus valores esperados, sob a suposição de normalidade, uma linha reta indicará que essa suposição é válida. Na modelagem de regressão logística, além de permitir a verificação da normalidade dos resíduos, esse gráfico pode também ser útil para diagnosticar a inadequação do modelo, falta de alguma informação na componente sistemática e a presença de pontos aberrantes. 
Entretanto, na modelagem em questão, os resíduos usados na construção do gráfico de probabilidades não serão necessariamente não-correlacionados e podem não ter distribuição aproximadamente normal, mesmo quando o modelo ajustado for correto. Por esse motivo, esse gráfico não apresentará necessariamente uma linha reta mesmo quando o modelo ajustado é de fato correto.

Assim, a interpretação do gráfico normal de probabilidades para os resíduos padronizados deve ser auxiliada pela construção de envelopes, que são bandas de confiança simuladas que cobrem os resíduos (Collet, 1990). Esses envelopes são tais que, se o modelo estiver bem ajustado, a maioria dos pontos "plotados" deve estar dentro dessas bandas.

Para construir esse gráfico, deve-se, inicialmente, simular, para a $i$-ésima observação, com $i=I, \ldots, k$, outras $m$ observações adicionais de uma distribuição binomial de parâmetros $n_{i} \mathrm{e}$ $\hat{h}_{i}$, onde no modelo estudado $n_{i}$ é o número de pacientes vivos no início do $i$-ésimo intervalo e $\hat{h}_{i}$ é a probabilidade condicional de morte ajustada para o $i$-ésimo intervalo. $O$ modelo ajustado para os dados originais é então ajustado para cada um dos $m$ conjuntos de valores simulados da variável resposta binomial, onde as variáveis exploratórias do modelo assumem seus valores originais.

Para cada um desses ajustes, os valores dos resíduos $t_{D_{i}}$ padronizados são obtidos e ordenados. A média, mínimo e máximo desses resíduos, em cada intervalo, são "plotados" com os resíduos do conjunto de dados original.

Usando 19 simulações, quantidade sugerida por Atkinson (1985), existe 1 chance em 20 ou $5 \%$ de que o maior valor absoluto dos resíduos do conjunto de dados originais esteja fora dos envelopes simulados, quando o modelo ajustado for o correto. Esse resultado pode ser usado para detectar a presença de pontos aberrantes.

Segue a seguir um esquema simplificado da construção dos envelopes simulados para efeito de visualização.

Como já foi dito anteriormente, deve-se gerar uma amostra de tamanho 19, por exemplo, de uma distribuição $\operatorname{bin}\left(n_{i} ; \hat{h}_{i}\right)$ para o $i$-ésimo intervalo, $i=I, \ldots, k$, obtendo-se assim, 19 conjuntos de observações simuladas, como no esquema apresentado na Figura 2.1a. 


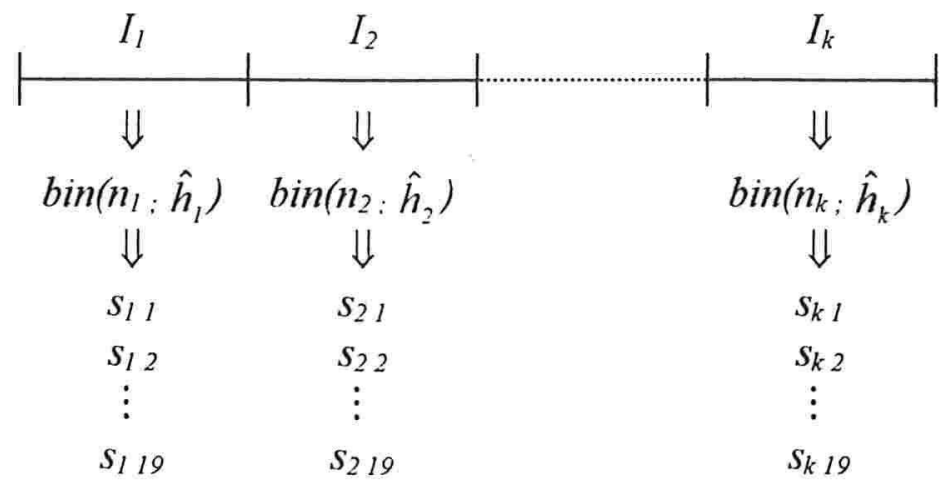

Figura 2.1a: Esquematização para construção dos envelopes simulados.

Para cada um dos 19 conjuntos de dados formados $\left(s_{1 j}, s_{2 j}, \ldots, s_{k j}\right), j=1, \ldots, 19$, ajustase um modelo com as mesmas variáveis explicativas do modelo original, obtendo-se, assim, os resíduos $t_{D_{i}}$ padronizados, denotado agora por $r_{i}$, como indicado na Figura 2.1b.

$$
\begin{array}{cccccc}
\left(s_{11}, s_{21}, \ldots, s_{k 1}\right) & \Rightarrow & r_{11} & r_{21} & \ldots & r_{k 1} \\
\left(s_{12}, s_{22}, \ldots, s_{k 2}\right) & \Rightarrow & r_{12} & r_{22} & \ldots & r_{k 2} \\
\vdots & & \vdots & \vdots & & \vdots \\
\left(s_{119}, s_{219}, \ldots, s_{k 19}\right) & \Rightarrow & r_{119} & r_{219} & \ldots & r_{k 19}
\end{array}
$$

Figura 2.1b: Obtenção do resíduos padronizados a partir do ajuste dos 19 conjuntos de dados.

Em seguida, ordena-se os resíduos obtidos,

$$
\begin{gathered}
r_{(1) 1}<r_{(2) 1}<\ldots<r_{(k) 1} \\
r_{(1) 2}<r_{(2) 2}<\ldots<r_{(k) 2} ; \\
\vdots \quad \vdots \quad \vdots \\
r_{(1) 19}<r_{(2) 19}<\ldots<r_{(k) 19} ;
\end{gathered}
$$


e por fim toma-se a média, mínimo e máximo de cada um dos intervalos:

$$
\begin{gathered}
r_{(1) M} \leq r_{(2) M} \leq \ldots \leq r_{(k) M} \\
r_{(1) I} \leq r_{(2) I} \leq \ldots \leq r_{(k) I} \\
r_{(1) S} \leq r_{(2) S} \leq \ldots \leq r_{(k) S},
\end{gathered}
$$

onde $r_{(i) M}=\underset{1 \leq j \leq 19}{=\operatorname{med}} r_{(i) j}, \quad r_{(i) 1} \underset{1 \leq j \leq 19}{=\min _{1 i}} r_{(i) j} \quad \mathrm{e} \quad r_{(i) S}=\underset{1 \leq j \leq 19}{\max } r_{(i) j}$

Na prática o número de envelopes gerados é maior que 19. Nos exemplos apresentados no texto será usado $m=100$ e será considerado bandas de confiança de $95 \%$.

A suspeita de que a $i$-ésima observação seja um ponto aberrante, pode ser verificada também ajustando o modelo $\log i t(h)=X \alpha+\lambda_{i} \boldsymbol{u}_{i}$, onde $\boldsymbol{u}_{i}$ é um vetor com $k$ - 1 zeros e um na $i$-ésima posição, e testando a hipótese de que $\lambda_{i}=0$. Para tanto, deve-se ajustar o modelo completo (com todas as observações) e comparar com o modelo sem a $i$-ésima observação através da estatística da razão de verossimilhança, denominada nesse caso particular de $\varepsilon_{G_{i}}$. Nesse contexto, pode-se ainda ter interesse em testar a hipótese de que existe uma única observação que é ponto aberrante, cuja identidade é desconhecida, e nesse caso uma estatística natural seria o máximo entre as estatísticas $\varepsilon_{G_{i}}$ 's. Entretanto, o cálculo dessa estatística exigiria $k+l$ ajustes não sendo, portanto, interessante do ponto de vista computacional. Williams (1986) estabeleceu, então, uma aproximação para o máximo das estatísticas $\varepsilon_{G_{i}}$ 's dada por

$$
t_{G_{i}}^{2}=\left(1-p_{i}\right) t_{D_{i}}^{2}+p_{i} t_{S_{i}}^{2},
$$


que pode, portanto, ser utilizada para testar a presença de um único ponto aberrante. A distribuição assintótica dessa estatística é uma qui-quadrado com um grau de liberdade, somente quando a distribuição da variável resposta é normal ou converge para ela.

Considerando $t_{G_{i}}=\sqrt{\left(1-p_{i}\right) t_{D_{i}}^{2}+p_{i} t_{S_{i}}^{2}}$ com o sinal de $\left(s_{i}-n_{i} \hat{h}_{i}\right)$, obtém-se um resíduo, que é uma combinação linear dos resíduos $t_{D_{i}}^{2}$ e $t_{S_{i}}^{2}$. Williams constatou através de simulações que esse resíduo possui uma média ligeiramente diferente de zero, variância excedendo um e uma leve assimetria, mas ainda assim, pode ser uma boa escolha quando se tem um interesse maior na deteç̧ão de "pontos aberrantes". Da mesma forma que os outros tipos de resíduo o gráfico contra valores ajustados pode ser bastante informativo. Além disso, a interpretação do gráfico normal de probabilidades pode também ser auxiliada pela adição de envelopes.

\subsection{Superdispersão}

Quando um modelo de regressão logística é utilizado, ele deve ajustar adequadamente as probabilidades de resposta observadas e, adicionalmente, modelar de maneira apropriada a variação aleatória dos dados. Quando a variabilidade do número de sucessos é maior do que a variabilidade dessa quantidade obtida no modelo em que a suposição de binomiais independentes é válida, ocorre um fenômeno denominado superdispersão.

A superdispersão pode ocorrer quando, por exemplo, a componente sistemática não foi corretamente especificada, quando a função de ligação entre a média e o preditor linear é incorreta ou ainda na presença de pontos aberrantes, e pode ser explicada pela ocorrência de variação entre as probabilidades de resposta ou correlação entre as respostas binárias, em outras palavras, correlação entre as réplicas da binomial. Essas duas causas representam na verdade o mesmo problema, pois correlação entre respostas binárias acarreta variação entre as probabilidades de resposta e vice-versa. 
No caso da análise de sobrevivência, suponha que se tenha interesse em estudar, por exemplo, a sobrevida de pacientes com uma certa doença genética. Nesse caso, mesmo quando os indivíduos são expostos às mesmas condições no experimento essa influência genética pode acarretar uma variação na probabilidade de morte de cada individuo. Portanto, mesmo em análise de sobrevivência, a aplicação da regressão logística pode apresentar a superdispersão e nesse caso ela deve ser modelada.

O fenômeno da variação entre as probabilidades de resposta acontece quando grupos expostos a condições similares no experimento possuem probabilidades de resposta diferentes, e pode ser causada pela falta de alguma informação no modelo que seja relevante ou pela existência de alguma variável explicativa importante que não foi controlada ou medida adequadamente.

Já a correlação entre as réplicas da binomial acontece quando a suposição de independência entre as respostas binárias não é válida. Desde que o número de sucessos segue uma distribuição binomial somente quando as componentes binárias são independentes, a nãoindependência levará a uma variabilidade maior ou menor do que o caso em que essa independência existe.

No primeiro caso, em que as probabilidades de resposta variam entre as unidades experimentais expostas às mesmas condições, algumas suposições devem ser feitas sobre a forma dessa variação. Considere o caso em que $k$ proporções são observadas e suponha que a probabilidade de resposta para a $i$-ésima unidade experimental dependa de $p$ variáveis explicativas, segundo um modelo logístico. Para introduzir variabilidade na probabilidade de resposta da $i$-ésima observação, deve-se assumir que a verdadeira probabilidade de resposta, $\pi_{i}$, varia em torno de uma média $h_{i}$, ou seja, $\mathrm{E}\left\{\pi_{i}\right\}=h_{i}$. Portanto, $\pi_{i}$ deve ser considerada uma variável aleatória. Como trata-se de uma probabilidade, a variância de $\pi_{i}$ deve ser zero quando $h_{i}$ é zero ou um, e sendo assim, a função que descreve essa variabilidade pode ser da forma

$$
\operatorname{Var}\left(\pi_{i}\right)=\phi h_{i}\left(1-h_{i}\right),
$$


onde $\phi \geq 0$ é um parâmetro de escala a ser estimado. A quantidade $\pi_{i}$ é uma variável aleatória não-observável. Entretanto, dado um particular valor de $\pi_{i}$, o número de sucessos terá uma distribuição binomial com média $n_{i} \pi_{i}$ e variância $n_{i} \pi_{i}\left(l-\pi_{i}\right)$. Em outras palavras, a média e variância do número de sucessos condicionado à verdadeira probabilidade de resposta $\pi_{i}$ são dadas, respectivamente, por

$$
E\left\{s_{i} \mid \pi_{i}\right\}=n_{i} \pi_{i}
$$

e

$$
\operatorname{Var}\left\{s_{i} \mid \pi_{i}\right\}=n_{i} \pi_{i}\left(1-\pi_{i}\right)
$$

Desde que $\pi_{i}$ não pode ser estimado, a proporção $s_{i} / n_{i}$ deve ser tomada como uma estimativa de $h_{i}$, que é o valor esperado de $\pi_{i}$. Assim, para investigar o efeito da suposição de variação nas probabilidades de resposta no valor esperado e variância do número de sucessos deve-se obter as estimativas incondicionais dessas quantidades.

De acordo com o teorema da probabilidade condicional, a esperança e variância incondicionais do número de sucessos, são dadas, respectivamente, por

$$
\begin{aligned}
E\left\{s_{i}\right\} & =E\left\{E\left\{s_{i} \mid \pi_{i}\right\}\right\} \\
& =E\left\{n_{i} \pi_{i}\right\} \\
& =n_{i} h_{i}
\end{aligned}
$$




$$
\begin{aligned}
\operatorname{Var}\left\{s_{i}\right\} & =E\left\{\operatorname{Var}\left\{s_{i} \pi_{i}\right\}\right\}+\operatorname{Var}\left\{E\left\{s_{i} \mid \pi_{i}\right\}\right\} \\
& =E\left\{n_{i} \pi_{i}\left(1-\pi_{i}\right)\right\}+\operatorname{Var}\left\{n_{i} \pi_{i}\right\} \\
& =n_{i}\left[E\left\{\pi_{i}\right\}-E\left\{\pi_{i}^{2}\right\}\right]+n_{i}^{2} \operatorname{Var}\left\{\pi_{i}\right\} \\
& =n_{i}\left[h_{i}-\operatorname{Var}\left\{\pi_{i}\right\}-E^{2}\left\{\pi_{i}\right\}\right]+n_{i}^{2} \phi h_{i}\left(1-h_{i}\right) \\
& =n_{i}(1-\phi) h_{i}\left(1-h_{i}\right)+n_{i}^{2} \phi h_{i}\left(1-h_{i}\right) \\
& =n_{i} h_{i}\left(1-h_{i}\right)\left\{\left(1+\phi\left(n_{i}-1\right)\right\}\right. \\
& =n_{i} h_{i}\left(1-h_{i}\right) \sigma_{i}^{2} .
\end{aligned}
$$

$\mathrm{Na}$ ausência de variação nas probabilidades de resposta, ou seja, quando $\phi=0$, o número de sucessos, $s_{i}$, tem distribuição $\operatorname{bin}\left(n_{i} ; h_{i}\right)$ e, nesse caso, $\operatorname{Var}\left\{s_{i}\right\}=n_{i} h_{i}\left(I-h_{i}\right)$. Se $\phi \geq 0$ então existem indícios de superdispersão.

Para entender o segundo caso em que existe correlação entre as réplicas da binomial, considere o problema estudado onde se observa o número de sucessos (morte) $s_{i}$, entre os indivíduos vivos no início do $i$-ésimo intervalo, $n_{i}$. Considere que associado a cada indivíduo no $i$-ésimo intervalo exista uma variável binária $R_{i j}, j=1, \ldots, n_{i}$, que assume dois valores, sucesso (morte) ou fracasso. Nesse contexto, $R_{i j}$ segue uma distribuição Bernoulli de parâmetro $h_{i}$ e, portanto, $E\left\{R_{i j}\right\}=h_{i}$ e $\operatorname{Var}\left\{R_{i j}\right\}=h_{i}\left(1-h_{i}\right)$. Assim, o valor esperado e a 
variância do número de sucessos no $i$-ésimo intervalo, obtido através de $s_{i}=\sum_{j=1}^{n_{i}} R_{i j}$, são dados por

$$
E\left\{s_{i}\right\}=n_{i} h_{i}
$$

e

$$
\begin{aligned}
\operatorname{Var}\left\{s_{i}\right\} & =\sum_{j=1}^{n_{i}} \operatorname{Var}\left(R_{i j}\right)+\sum_{j=1}^{n_{i}} \sum_{j \neq k} \operatorname{cov}\left(R_{i j}, R_{i k}\right) \\
& =n_{i} h_{i}\left(1-h_{i}\right)+\sum_{j=1}^{n_{i}} \sum_{j \neq k} \operatorname{cov}\left(R_{i j}, R_{i k}\right) .
\end{aligned}
$$

Se as variáveis binárias $R_{i j}^{\prime} s, j=1, \ldots, n_{i}$, não forem mutuamente independentes, ou seja, se existir alguma relação entre o sucesso (morte) dos indivíduos presentes no estudo no $i$ ésimo intervalo, essa covariância não será igual a zero.

Considere, então, um modelo em que a correlação entre dois quaisquer $R_{i j}^{\prime} s$ é constante, ou seja, a correlação é igual a $\delta$ e, portanto, a covariância igual a $\delta h_{i}\left(1-h_{i}\right)$. Nesse caso, a variância do número de sucessos é dada por

$$
\begin{aligned}
\operatorname{Var}\left(s_{i}\right) & =n_{i} h_{i}\left(1-h_{i}\right)+n_{i}\left(n_{i}-1\right) \delta h_{i}\left(I-h_{i}\right) \\
& =n_{i} h_{i}\left(1-h_{i}\right)\left[1+\delta\left(n_{i}-1\right)\right] \\
& =n_{i} h_{i}\left(1-h_{i}\right) \sigma_{i}^{2} .
\end{aligned}
$$


Assim, novamente, quando $\delta>0$, tem-se $\operatorname{Var}\left(s_{i}\right)>n_{i} h_{i}\left(1-h_{i}\right)$, indicando a ocorrência de superdispersão.

A diferença entre os modelos de superdispersão nos dois casos estudados aqui consiste no fato de que no primeiro caso, $\phi$ é suposto maior ou igual a zero, já $\delta$ pode ser negativo. Entretanto, no segundo caso, como a quantidade $\left[1+\delta\left(n_{i}-1\right)\right]$ deve ser maior ou igual a zero, tem-se que $-1 /\left(n_{i}-1\right) \leq \delta \leq 1$. A menos que $n_{i}$ seja muito pequeno, o limite inferior para $\delta$ será efetivamente o zero.

Assim, tanto no primeiro quanto no segundo caso o modelo de superdispersão é o mesmo de onde se conclui que não é possível distinguir se a superdispersão é devido ao fato de existir variação entre as probabilidades de resposta ou correlação entre as respostas binárias.

Existem várias maneiras de contornar o problema da superdispersão. Morgan (1992, Cap.6) discute o ajuste do modelo de superdispersão usando o método de quasiverossimilhança a partir do qual é possível estimar um parâmetro de dispersão $\sigma^{2}$, que é assumido constante para os $k$ intervalos. Esse método é uma alternativa quando as informações para construção da verossimilhança são insuficientes. A idéia básica consiste em construir a verossimilhança para uma variável aleatória $Y$, cuja média é dada por $E(Y)=\mu$, considerando que sua variância assuma a forma

$$
\operatorname{Var}(Y)=\sigma^{2} V(\mu)
$$

onde $V(\mu)$ é a função de variância associada à essa variável aleatória. Nessas condições define-se a função

$$
U(\mu, y)=\frac{Y-\mu}{\sigma^{2} V(\mu)},
$$


que possui algumas propriedades encontradas nas funções escores como:

$$
\begin{gathered}
E(U)=0, \\
\operatorname{Var}(U)=\frac{1}{\sigma^{2} V(\mu)} \mathrm{e} \\
-E\left(\frac{\partial U}{\partial \mu}\right)=\frac{1}{\sigma^{2} V(\mu)} .
\end{gathered}
$$

Por esse motivo pode-se inferir que a integral

$$
Q(\mu, y)=\int_{y}^{\mu} \frac{Y-t}{\sigma^{2} V(t)} d t
$$

se existir, deve apresentar um comportamento semelhante ao do logaritmo da função de verossimilhança para $\mu$, sendo denominada, portanto, de função de quasi-verossimilhança. No caso particular em que a função de variância $V(\mu)$ é referente à alguma distribuição da família exponêncial, $Q(\mu, y)$ coincide com o logaritmo da função de verossimilhança dessa distribuição, apresentando, assim, as mesmas estimativas pontuais dos parâmetros, mas com a variabilidade inflacionada pelo parâmetro de dispersão. MacCullagh e Nelder (1989) apontam que a matriz de covariância dos parâmetros também deve ser corrigida $\left(\operatorname{Cov}(\hat{\alpha})=\sigma^{2} I^{-1}(\alpha)\right)$.

Uma outra maneira de contornar o problema da superdispersão, também discutida por Morgan (1992, Cap.6), consiste em modelar a variabilidade da verdadeira probabilidade de resposta no $i$-ésimo intervalo, $\pi_{i}$, com uma distribuição beta $\left(a_{i}, b_{i}\right)$, de tal forma a obter uma distribuição beta-binomial para o número de sucessos.

O fenômeno descrito aqui pode ser detectado através da função desvio, pois valores muito altos dessa medida indicam que o modelo não está bem ajustado, e uma possivel causa é 
a superdispersão, entretanto, a suspeita deve ser confirmada através do gráfico dos resíduos padronizados com envelopes. A superdispersão ocorre quando os resíduos negativos estão mais próximos da banda inferior e os resíduos positivos mais próximos da banda superior, pois esse comportamento indica que a variância do número de sucessos, utilizada na construção dos resíduos, é menor do que deveria ser, sugerindo uma correção através da divisão dos resíduos por um parâmetro de dispersão.

\subsection{Paralelo entre estimação paramétrica e não-paramétrica}

Como já foi visto, o estimador de Kaplan-Meier para a taxa de risco é definido por $\widetilde{h}_{i}=s_{i} / n_{i}$, sendo essa estimativa sempre não-viesada para $n_{i}>0$. Entretanto, se a suposição feita no modelo paramétrico, $s_{i} \mid n_{i} \sim \operatorname{bin}\left(n_{i}, h_{i}\right)$ independentes, para $i=1, \ldots, k$, for correta, a estimação paramétrica pode produzir melhores resultados. Para ilustrar, considere a razão das variâncias assintóticas dos estimadores paramétrico e não-paramétrico

$$
\begin{aligned}
\frac{\operatorname{Var}\left(\hat{h}_{i}\right)}{\operatorname{Var}\left(\widetilde{h}_{i}\right)} & =\frac{\left(h_{i}\left(1-h_{i}\right)\right)^{2} \boldsymbol{x}_{i}^{T} I^{-1}(\alpha) x_{i}}{h_{i}\left(1-h_{i}\right) / n_{i}} \\
& =n_{i} V_{i} x_{i}^{T} I^{-1}(\alpha) x_{i} \\
& =n_{i} V_{i} x_{i}^{T}\left(X^{T} \operatorname{diag}\left(n_{i} V_{i}\right) X\right)^{-1} x_{i} \\
& =n_{i} V_{i} x_{i}^{T}\left(\sum_{j=1}^{k} x_{i} n_{i} V_{i} x_{i}^{T}\right)^{-1} x_{i} \\
& =\sqrt{n_{i} V_{i}} x_{i}^{T}\left(\sum_{j=1}^{k} \sqrt{n_{i} V_{i}} x_{i} \sqrt{n_{i} V_{i}} x_{i}^{T}\right)^{-1} \sqrt{n_{i} V_{i}} x_{i} .
\end{aligned}
$$


Portanto, a razão das variâncias assintóticas dos estimadores paramétrico e não-paramétrico é dada por

$$
\frac{\operatorname{Var}\left(\hat{h}_{i}\right)}{\operatorname{Var}\left(\tilde{h}_{i}\right)}=\boldsymbol{z}_{i}^{T}\left(\sum_{j=1}^{k} z_{j} z_{j}^{T}\right)^{-1} \boldsymbol{z}_{i},
$$

onde $z_{i}=x_{i} \sqrt{n_{i} V_{i}}$. Efron (1988) ressalta que, como essa razão é sempre menor que 1, a variância assintótica do estimador paramétrico é sempre menor que a variância do estimador não-paramétrico. Desse resultado segue o teorema:

Teorema 2.1. A média das razões das variâncias assintóticas dos estimadores paramétrico e não-paramétrico é dada por

$$
\frac{l}{k} \sum_{i=1}^{k} \frac{\operatorname{Var}\left(\hat{h}_{i}\right)}{\operatorname{Var}\left(\widetilde{h}_{i}\right)}=\frac{p}{k}
$$

Para verificar esse resultado basta notar que a razão das variâncias assintóticas dos estimadores paramétrico e não-paramétrico no $i$-ésimo intervalo é igual ao $i$-ésimo elemento da diagonal principal da matriz de projeção ortogonal $P, p_{i i}=n_{i} h_{i}\left(1-h_{i}\right) \boldsymbol{x}_{i}^{T}\left(\boldsymbol{X}^{T} \hat{\boldsymbol{V} X}\right)^{-1} \boldsymbol{x}_{i}$. Como a soma dos elementos da diagonal principal dessa matriz é igual a $p$, número de parâmetros do modelo, segue o resultado acima.

Note que, quanto mais parâmetros forem utilizados na regressão logística parcial, menor a vantagem de $\hat{h}_{i}$ sobre $\widetilde{h}_{i}$, e mais ainda, se $p=k$ então $\hat{h}_{i}=\widetilde{h}_{i}$. Portanto, a variabilidade de $\hat{h}_{i}$ obtida na regressão logística parcial aproxima-se da variabilidade do estimador de Kaplan-Meier, $\tilde{h}_{\imath}$, quando $p$ aproxima-se de $k$.

Para ilustrar esse fato, considere a variância da função de sobrevivência, já calculada na Seção 2.1.2. 


$$
\operatorname{Var}\left(\hat{G}_{i}\right)=G_{i}^{2}\left\{\left(\sum_{j=1}^{i} h_{j} x_{j}\right)^{T} I^{-1}(\alpha)\left(\sum_{j=l}^{i} h_{j} x_{j}\right)\right\}
$$

Essa fórmula está diretamente relacionada à fórmula de Greenwood para a variância do estimador de Kaplan-Meier. Considere, por exemplo, a situação em que $p=k$, ou seja, o número de parâmetros da regressão logística parcial é igual ao número de intervalos considerados. Nesse caso o vetor $\boldsymbol{x}_{\boldsymbol{i}}$ fica expresso por

$$
x_{i}=\left(\begin{array}{lll}
0 & 0 \ldots 1 \ldots 0
\end{array}\right)^{T}, \text { para } i=1, \ldots, k \text {, }
$$

onde o valor 1 encontra-se na $i$-ésima posição, e, portanto, a matriz modelo é uma matriz identidade de ordem $k, \boldsymbol{X}=\boldsymbol{I}_{K}$. Nesse caso, o estimador de máxima verossimilhança $\hat{\alpha}$, obtido igualando a equação $U(\alpha)=\boldsymbol{X}^{T}(\boldsymbol{s}-\boldsymbol{n h})$ a zero, assume o valor

$$
\hat{h}_{i}=\tilde{h}_{i}=\frac{s_{i}}{n_{i}}, \text { para } i=I, \ldots, k
$$

Portanto, o estimador de máxima verossimilhança da função de sobrevivência $\hat{G}_{i}$, nesse caso particular, é também igual ao de Kaplan-Meier $\widetilde{G}_{i}$. Além disso, a matriz de informação de Fisher pode ser facilmente obtida, sendo dada por $I(\alpha)=\operatorname{diag}\left(n_{i} V_{i}\right)$. Assim, a variância da função de sobrevivência fica expressa na forma

$$
\operatorname{Var}\left(\hat{G}_{t}\right)=G_{i}^{2}\left\{\left(\sum_{j=1}^{i} h_{j} x_{j}\right)^{T}\left[\operatorname{diag}\left\{n_{i} h_{i}\left(1-h_{i}\right)\right\}\right]^{-1}\left(\sum_{j=1}^{i} h_{j} x_{j}\right)\right\}
$$




$$
\begin{aligned}
& =G_{i}^{2}\left\{\left(h_{1}, h_{2} \cdots, h_{i}, 0, \cdots, 0\right)\left[\operatorname{diag}\left\{n_{i} h_{i}\left(1-h_{i}\right)\right\}\right]^{-1}\left(\begin{array}{c}
h_{1} \\
h_{2} \\
\vdots \\
h_{i} \\
0 \\
\vdots \\
0
\end{array}\right)\right\} \\
& =G_{i}^{2} \sum_{j=1}^{i} \frac{h_{j}^{2}}{n_{j} h_{j}\left(1-h_{j}\right)},
\end{aligned}
$$

sendo estimada por

$$
\hat{\operatorname{Var}}\left(\hat{G}_{i}\right)=\hat{G}_{i}^{2} \sum_{j=1}^{i} \frac{s_{j}}{n_{j}\left(n_{j}-s_{j}\right)} .
$$

Essa, como já foi visto no Capítulo 1, é conhecida como a fórmula de Greenwood, e, portanto, a variabilidade da função de sobrevivência $\hat{G}_{i}$ obtida com $p$ parâmetros da regressão logística parcial também tende para a variabilidade do estimador de Kaplan-Meier $\widetilde{G}_{i}$ quando $p \rightarrow k$.

\subsection{Relação entre o modelo condicional e não-condicional}

Ao utilizar a regressão logística parcial, assume-se, como já foi dito, binomiais independentes em cada intervalo baseado no condicionamento do número de indivíduos em risco e, portanto, trata-se de um modelo condicional. Entretanto, essa suposição pode não ser verdadeira. No caso sem censura, por exemplo, o número de mortes no $i$-ésimo intervalo pode ser obtido através da diferença entre o número de indivíduos em risco no início do $i$-ésimo e (i+l)-ésimo intervalo, $s_{i}=n_{i}-n_{i+1}$. Portanto, a sequência $s_{l}, s_{2}, \ldots, s_{k}$ é completamente 
determinada por $n_{l}, n_{2}, \ldots, n_{k}$, contradizendo, então, a suposição de independência. Ainda assim, existem boas razões para se utilizar o modelo condicional.

Considere o modelo não-condicional, ou completo, em que a informação da censura é incorporada. Usando a notação já apresentada, seja $v_{i}=\left(s_{1}, s_{1}{ }^{\prime}, s_{2}, s_{2}{ }^{\prime} \ldots, s_{i-1}, s_{i-1}{ }^{\prime}\right)$ e $v_{i}{ }^{\prime}=\left(s_{1}, s_{1}{ }^{\prime}, s_{2}, s_{2}{ }^{\prime} \ldots, s_{i-1}, s_{i-1}{ }^{\prime}, s_{i}\right)$, de tal forma que $v_{i}$ representa a história de mortes e censuras até o (i-1)-ésimo intervalo e $v_{i}{ }^{\prime}$ a história de mortes e censuras incluindo a informação das mortes no $i$-ésimo intervalo. Considerando somente o número de indivíduos em risco no início do seguimento $n_{1}$, toda sequência $n_{2}, \ldots, n_{i}$ pode ser determinada através de $n_{l}$ e $v_{i}$ (ou $v_{i}{ }^{\prime}$ ). Assuma, também, que o número de mortes no $i$-ésimo intervalo dado toda a história anterior, ou seja, $s_{i}$ dado $v_{i}$, segue uma distribuição $\operatorname{bin}\left(n_{i}, h_{i}\right)$ e que, o número de censuras no $i$-ésimo intervalo dado toda a história anterior, ou seja, $s_{i}{ }^{\prime}$ dado $v_{i}{ }^{\prime}$, segue uma distribuição $f_{\varepsilon}\left(s_{i}{ }^{\prime} \mid \boldsymbol{v}_{i}{ }^{\prime}\right)$ que depende de um parâmetro $\varepsilon$, denominado parâmetro de perturbação. É razoável admitir que $f_{\varepsilon}\left(s_{i}{ }^{\prime} \mid \boldsymbol{v}_{i}{ }^{\prime}\right)$ não depende de $\alpha$, já que as censuras não dependem do tempo, ou seja, acontecem de maneira aleatória ao longo do intervalo considerado. Assim, a distribuição conjunta das mortes e censuras para todo o intervalo de tempo considerado é dada por

$$
\begin{aligned}
f_{\alpha, \varepsilon}\left(s_{1}, s_{1}{ }^{\prime}, s_{2}, s_{2}{ }^{\prime}, \ldots, s_{k}, s_{k}{ }^{\prime}\right) & =f_{\alpha, \varepsilon}\left(s_{k}, s_{k}{ }^{\prime}, v_{k}\right) \\
& =f_{\alpha, \varepsilon}\left(s_{k}, s_{k}{ }^{\prime} \mid, v_{k}\right) f_{\alpha, \varepsilon}\left(v_{k} \mid v_{k-1}\right) f_{\alpha, \varepsilon}\left(v_{k-1} \mid v_{k-2}\right) \ldots f_{\alpha, \varepsilon}\left(v_{2} \mid v_{l}\right)
\end{aligned}
$$

mas,

$$
\begin{aligned}
f_{\alpha, \varepsilon}\left(s_{k}, s_{k}{ }^{\prime} \mid v_{k}\right) & =f_{\alpha, \varepsilon}\left(s_{k}, s_{k}{ }^{\prime} \mid s_{1}, s_{1}{ }^{\prime}, s_{2}, s_{2}{ }^{\prime} \ldots, s_{k-1}, s_{k-1}{ }^{\prime}\right) \\
& =f_{\alpha, \varepsilon}\left(s_{k}{ }^{\prime} s_{l}, s_{l}{ }^{\prime}, s_{2}, s_{2}{ }^{\prime} \ldots, s_{k-1}, s_{k-1}{ }^{\prime}, s_{k}\right) f_{\alpha, \varepsilon}\left(s_{k} \mid s_{1}, s_{1}{ }^{\prime}, s_{2}, s_{2}{ }^{\prime} \ldots, s_{k-1}, s_{k-1}{ }^{\prime}\right) \\
& =f_{\alpha, \varepsilon}\left(s_{k}{ }^{\prime} v_{k}{ }^{\prime}\right) f_{\alpha, \varepsilon}\left(s_{k} \mid v_{k}\right),
\end{aligned}
$$


e no caso geral

$$
\begin{aligned}
f_{\alpha, \varepsilon}\left(v_{i} v_{i-1}\right) & =f_{\alpha, \varepsilon}\left(s_{1}, s_{1}{ }^{\prime}, s_{2}, s_{2}{ }^{\prime} \ldots, s_{i-1}, s_{i-1}{ }^{\prime} \mid s_{1}, s_{1}{ }^{\prime}, s_{2}, s_{2}{ }^{\prime} \ldots, s_{i-2}, s_{i-2}{ }^{\prime}\right) \\
& =f_{\alpha, \varepsilon}\left(s_{i-1}, s_{i-1}{ }^{\prime} \mid s_{1}, s_{1}{ }^{\prime}, s_{2}, s_{2}{ }^{\prime} \ldots, s_{i-2}, s_{i-2}{ }^{\prime}\right) \\
& =f_{\alpha, \varepsilon}\left(s_{i-1}{ }^{\prime} s_{1}, s_{1}{ }^{\prime}, s_{2}, s_{2}{ }^{\prime} \ldots, s_{i-2}, s_{i-2}{ }^{\prime}, s_{i-1}\right) f_{\alpha, \varepsilon}\left(s_{i-1} \mid s_{1}, s_{1}{ }^{\prime}, s_{2}, s_{2}{ }^{\prime} \ldots, s_{i-2}, s_{i-2}{ }^{\prime}\right) \\
& =f_{\alpha, \varepsilon}\left(s_{i-1}{ }^{\prime} \mid v_{i-1}{ }^{\prime}\right) f_{\alpha, \varepsilon}\left(s_{i-1} \mid v_{i-1}\right) .
\end{aligned}
$$

Portanto, a distribuição conjunta das mortes e censuras, ou verossimilhança do modelo nãocondicional, pode ser escrita na forma

$$
f_{\alpha, \varepsilon}\left(s_{l}, s_{1}{ }^{\prime}, s_{2}, s_{2}{ }^{\prime} \ldots, s_{k}, s_{k}{ }^{\prime}\right)=f_{\alpha, \varepsilon}\left(s_{k} \mid v_{k}\right) f_{\alpha, \varepsilon}\left(s_{k}{ }^{\prime} \mid v_{k}{ }^{\prime}\right) \ldots f_{\alpha, \varepsilon}\left(s_{l} \mid v_{l}\right) f_{\alpha, \varepsilon}\left(s_{1}{ }^{\prime} \mid v_{l}{ }^{\prime}\right),
$$

ou ainda

$$
f_{a, \varepsilon}\left(s_{1}, s_{1}{ }^{\prime}, s_{2}, s_{2}{ }^{\prime} \ldots, s_{k}, s_{k}{ }^{\prime}\right)=\prod_{i=1}^{k}\left(\begin{array}{c}
n_{i} \\
s_{i}
\end{array}\right) h_{i} s_{i}\left(1-h_{i}\right)^{n_{i}-s_{i}} \prod_{i=1}^{k} f_{\alpha, \varepsilon}\left(s_{i}{ }^{\prime} \mid v_{i}{ }^{\prime}\right) .
$$

Tomando o logaritmo dessa função tem-se

$$
\log f_{\alpha, \varepsilon}\left(s_{1}, s_{1}{ }^{\prime}, s_{2}, s_{2}{ }^{\prime} \ldots, s_{k}, s_{k}{ }^{\prime}\right)=L_{\alpha, \varepsilon}=L_{\alpha}+L_{\varepsilon},
$$

onde $L_{\alpha}=\sum_{i=1}^{k} \log \left\{\left(\begin{array}{c}n_{i} \\ s_{i}\end{array}\right) h_{i} s_{i}\left(1-h_{i}\right)^{n_{i}-s_{i}}\right\}$ e $L_{\varepsilon}$ não depende de $\alpha$. Portanto, é fácil notar que o modelo condicional estudado é uma parte do modelo completo, o que explica o fato da técnica em estudo ser denominada regressão logística parcial. Efron comenta que esse nome vem por analogia à verossimilhança parcial do modelo de Cox, onde a verossimilhança 
completa pode ser decomposta em duas partes tal que somente uma delas contém informação acerca do vetor de parâmetros de interesse. Assume-se, então, que a verossimilhança parcial se comporta como uma verossimilhança comum e estima-se o vetor de parâmetros normalmente.

Como $L_{\alpha}$ é o logaritmo da função de verossimilhança para a situação de $k$ binomiais independentes, como no modelo condicional, os seguintes fatos se seguem:

- O vetor escore para $\alpha$ no modelo não-condicional é o mesmo do modelo condicional, ou seja,

$$
U(\alpha)=\frac{\partial_{\alpha, \varepsilon}}{\partial \alpha}=\frac{\partial L_{\alpha}}{\partial \alpha}=X^{T}(\boldsymbol{s}-\boldsymbol{n h}),
$$

e consequentemente o estimador de máxima verossimilhança $\hat{\alpha}$ também.

- A matriz de segundas derivadas é bloco-diagonal

$$
-\frac{\partial^{2} L_{\alpha, \varepsilon}}{\partial \gamma \partial \gamma^{T}}=-\left(\begin{array}{cc}
\frac{\partial^{2} L_{\alpha, \varepsilon}}{\partial \alpha \partial \alpha^{T}} & 0 \\
0 & \frac{\partial^{2} L_{\alpha, \varepsilon}}{\partial \varepsilon \partial \varepsilon^{T}}
\end{array}\right)=-\left(\begin{array}{cc}
\frac{\partial^{2} L_{\alpha}}{\partial \alpha \partial \alpha^{T}} & 0 \\
0 & \frac{\partial^{2} L_{\varepsilon}}{\partial \varepsilon \partial \varepsilon^{T}}
\end{array}\right),
$$

onde $\gamma=(\alpha, \varepsilon)^{T}$ e $-\frac{\partial^{2} L_{\alpha, \varepsilon}}{\partial \alpha \partial \alpha^{T}}=X^{T} \operatorname{diag}\left(n_{i} V_{i}\right) X$ é a matriz de informação de Fisher observada que é igual a matriz de informação de Fisher observada e esperada no modelo condicional.

- A matriz de informação de Fisher esperada é 


$$
I(\alpha, \varepsilon)=E_{\alpha, \varepsilon}\left\{-\frac{\partial^{2} L_{\alpha, \varepsilon}}{\partial \gamma \partial \gamma^{T}}\right\}=\left(\begin{array}{cc}
E_{\alpha, \varepsilon}\left\{-\frac{\partial^{2} L_{\alpha}}{\partial \alpha \partial \alpha^{T}}\right\} & 0 \\
0 & E_{\alpha, \varepsilon}\left\{-\frac{\partial^{2} L_{\varepsilon}}{\partial \varepsilon \partial \varepsilon^{T}}\right\}
\end{array}\right)
$$

e portanto a matriz de variância-covariância assintótica de $\hat{\alpha}$ é obtida tomando o bloco pxp superior esquerdo da diagonal da matriz $I^{-1}(\alpha, \varepsilon)$, ou seja,

$$
\operatorname{Var}_{\alpha, \varepsilon}(\hat{\alpha})=\left(E_{\alpha, s}\left\{-\frac{\partial^{2} L_{\alpha}}{\partial \alpha \partial \alpha^{T}}\right\}\right)^{-1}
$$

Note que não necessariamente tem-se a igualdade

$$
E_{\alpha, \varepsilon}\left\{-\frac{\partial^{2} L_{\alpha}}{\partial \alpha \partial \alpha^{T}}\right\}=-\frac{\partial^{2} L_{\alpha}}{\partial \alpha \partial \alpha^{T}}
$$

como acontece no modelo condicional, pois no modelo completo $n_{i}$ também é variável aleatória. Assim, com o objetivo de comparar os valores esperados das matrizes de variânciacovariância de $\hat{\alpha}$ no modelo condicional e completo, considere a desigualdade de Jensen (ver por exemplo, Barry James, 1981) que diz que se $f(x)$ é uma função convexa, então

$$
f(E(x)) \leq E(f(x))
$$

Assim, utilizando a desigualdade de Jensen pode-se concluir que

$$
\left(E_{\alpha, \varepsilon}\left\{-\frac{\partial^{2} L_{\alpha}}{\partial \alpha \partial \alpha^{T}}\right\}\right)^{-1} \leq E_{\alpha, \varepsilon}\left\{-\frac{\partial^{2} L_{\alpha}}{\partial \alpha \partial \alpha^{T}}\right\}^{-1}
$$


Em resumo, o modelo condicional, baseado na suposição de binomiais independentes, fornece a mesma estimativa de máxima verossimilhança que o modelo completo. Além disso, a estimativa da matriz de variância-covariância de $\hat{\alpha}$ obtida no modelo condicional, baseada na matriz de informação de Fisher observada, não difere do modelo completo. Entretanto, isso não é verdade quando se considera a matriz de informação de Fisher esperada, e mais ainda, o resultado (2.5) sugere que em média a variância-covariância aproximada obtida no modelo de binomiais independentes é maior que a obtida no modelo completo.

\section{8. $O$ caso contínuo}

Em análise de sobrevivência clássica existem técnicas de modelagem do tempo de sobrevivência por algumas funções contínuas como exponencial, Weibull, lognormal entre outras. Entretanto, uma vez definida a distribuição do tempo de sobrevivência, a distribuição da função de risco fica pré-estabelecido. Resta somente verificar através de gráficos se é razoável aceitar que a função de risco assume a forma imposta pelo modelo.

Modelos contínuos para a função de risco podem ser obtidos, por exemplo, como um caso limite da regressão logística parcial quando o tamanho dos intervalos considerados tende a zero.

Considere $\Delta_{i}$ o tamanho do $i$-ésimo intervalo. Efron (1988) mostra que

$$
\log \left(\frac{h_{i, \alpha}}{\left(1-h_{i, \alpha}\right)}\right)=\log \left(\Delta_{i}\right)+\log \left(h_{\alpha}\left(t_{i}\right)\right)+1 / 2 h_{\alpha}\left(t_{i}\right) \Delta_{i}+O\left(\Delta_{i}\right)
$$

onde $h_{i, \alpha}$ é a função de risco discreta e $h_{\alpha}\left(t_{i}\right)=\exp \left(x\left(t_{i}\right) \alpha\right)$ é a função de risco contínua. Em particular, quando $\Delta=\Delta_{i}$ a equação acima reduz-se a 


$$
\log \left(\frac{h_{i, \alpha}}{\left(1-h_{i, \alpha}\right)}\right)=\log (\Delta)+\log \left(h_{\alpha}\left(t_{i}\right)\right)+O(\Delta)
$$

e, a medida que $\Delta \rightarrow 0$, tem-se que $O(\Delta) \rightarrow 0$ e a quantidade $\log (\Delta)$ é absorvida pela constante do modelo. Portanto a classe de modelos paramétricos contínuos para modelar a função de risco é da forma

$$
h_{\alpha}(t)=\exp \{x(t) \alpha\}
$$

onde $\alpha$ é um vetor de $p$ parâmetros desconhecidos e $\boldsymbol{x}(t)$ é um vetor $p$-dimensional referente à um polinômio do tempo. Por conveniência, assume-se que a primeira coordenada de $x(t)$ é 1 para todo $t$.

Considere então o caso mais simples, onde $p=1$, e, portanto, a função de risco é da forma

$$
h_{\alpha}(t)=\exp \left\{\alpha_{1}\right\}, \text { para todo } t>0
$$

Seja $\theta=\exp \left\{\alpha_{1}\right\}$. A função de risco acumulada, definida como $H_{\alpha}(t)=\int_{0}^{t} h_{\alpha}(s) d s$, é dada por $H_{\alpha}(t)=\theta t$ e, então, a função de sobrevivência, definida por $G_{\alpha}(t)=\exp \left(-H_{a}(t)\right)$, é dada por $G_{a}(t)=\exp (-\theta t)$. Essa é a função de sobrevivência referente a distribuição exponencial de parâmetro $\theta=\exp \left\{\alpha_{1}\right\}$ para o tempo. Assim, modelando a função de risco, como em (2.8), é possivel também determinar a distribuição do tempo de sobrevivência.

O caso em que o logaritmo da função de risco varia linearmente com o tempo é o próximo caso mais simples, expresso por

$$
h_{\alpha}(t)=\exp \left\{\alpha_{1}+\alpha_{2} t\right\}, \text { para todo } t>0
$$


A função de risco acumulada é dada por

$$
\begin{aligned}
H_{\alpha}(t) & =\int_{0}^{t} \exp \left\{\alpha_{1}+\alpha_{2} s\right\} d s \\
& =\frac{\exp \left\{\alpha_{1}+\alpha_{2} t\right\}}{\alpha_{2}}-\frac{\exp \alpha_{1}}{\alpha_{2}} \\
& =\frac{\exp \alpha_{1}}{\alpha_{2}}\left\{\exp \left\{\alpha_{2} t\right\}-1\right\} \\
& =\theta\left\{\exp \left\{\alpha_{2} t\right\}-1\right\},
\end{aligned}
$$

onde $\theta=\exp \left\{\alpha_{1}\right\} / \alpha_{2}$. Consequentemente, a função de sobrevivência é obtida através da expressão $G_{\alpha}(t)=\exp \left[-\theta\left\{\exp \left(\alpha_{2} t\right)-1\right\}\right]$ que é a função de sobrevivência referente a uma distribuição para o tempo também conhecida como função de sobrevivência de Gompertz (ver por exemplo Lee, 1980). Note que para $\alpha_{2}>0$ e $\mathrm{t} \rightarrow \infty$, a função $G_{\alpha}(t)$ aproxima-se de zero, enquanto que se $\alpha_{2}<0$ e $\mathrm{t} \rightarrow \infty, \lim _{t \rightarrow \infty} G_{\alpha}(t)=\exp \{\theta\}$, onde $\theta<0$. Portanto, com esse modelo é possível estimar $P\{T=\infty\}$ como uma probabilidade positiva, o que consiste em uma vantagem da metodologia apresentada aqui com relação aos modelos clássicos.

Exemplos mais complicados para o modelo (2.7), como o modelo quadrático, não resulta necessariamente em expressões simples ou conhecidas para a função de sobrevivência, mas esse fato é irrelevante desde que a estimação dos parâmetros do modelo proposto é feita através da função de risco.

\subsection{Como escolher a melhor "discretização" do tempo}

Como toda a metodologia de regressão logística parcial assume o tempo de seguimento dividido em intervalos, ou seja, discretizado, um importante aspecto que deve ser discutido é 
como escolher a melhor discretização. Efron comenta que quanto maior o número de intervalos considerados, menor a informação perdida devido a discretização. Entretanto, é necessário entender que a discretização deve ser feita ponderando-se o número de intervalos com um número de indivíduos em risco em cada intervalo suficientes para evidenciar alguma tendência. Assim, duas situações devem ser evitadas:

- um número de intervalos muito grande com poucos indivíduos em risco em cada intervalo;

- poucos intervalos com muitos indivíduos em risco em cada intervalo.

Essas duas situações não são adequadas para aplicar a técnica em questão pois, a primeira impossibilita a visualização de tendências relacionadas ao risco de morte e a segunda resulta em poucas observações, já que a observação na modelagem proposta é o intervalo. Portanto, como não há um critério definido para efetuar a discretização deve-se utilizar o bomsenso e ponderar as duas quantidades descritas anteriormente. Efron comenta também que propôs várias discretizações para o exemplo do câncer na cabeça e pescoço e constatou que pequenas alterações na discretização não afetam os resultados.

Ainda nesse contexto deve-se considerar o caso em que a discretização mais apropriada é baseada em intervalos de tamanhos desiguais. No problema do câncer na cabeça $\mathrm{e}$ pescoço, por exemplo, Efron utiliza para modelar o risco de morte no tratamento B uma discretização do tempo composta por intervalos de tamanho: 15 dias, 1 mês e dois meses. Nesse caso, pode-se utilizar um modelo ainda mais refinado que leva em conta o tamanho dos intervalos, através da suposição de que a chance de morte em cada intervalo é proporcional ao seu comprimento. Trata-se de uma suposição bastante razoável indicando que em um intervalo de comprimento duas unidades a chance de morte é duas vezes maior do que em um intervalo de comprimento uma unidade, ou seja, considerando $\Delta_{i}$ o comprimento do i-ésimo intervalo tem-se

$$
\frac{h_{i}}{\left(I-h_{i}\right)}=\Delta_{i} \exp \left(x_{i}^{T} \alpha\right)
$$

ou ainda, 


$$
\log \left(\frac{h_{i}}{\left(1-h_{i}\right)}\right)=\log \left(\Delta_{1}\right)+\exp \left(x_{i}^{T} \alpha\right) .
$$

Portanto, essa suposição implica em adicionar ao modelo original um "offset" ou seja, um termo constante relacionado ao comprimento de cada intervalo. Efron justifica a utilização do "offset" através da expressão 2.6 e portanto essa suposição é válida quando o tamanho dos intervalos é pequeno.

\subsection{O exemplo do câncer na cabeça e pescoço}

No problema discutido no Exemplo 1.2 de câncer na cabeça e pescoço, Efron sugere, para relacionar a taxa de risco discreta com o tempo, os seguintes modelos paramétricos: linear, cúbico e cúbico-linear "spline".

Se o objetivo do estudo for estudar o comportamento da taxa de risco discreta em cada tratamento sem necessariamente estabelecer comparações, modelos diferentes podem ser considerados. Assim, cada um dos modelos propostos foi ajustado separadamente para os tratamentos $\mathrm{A}$ e $\mathrm{B}$ de tal forma a permitir que a taxa de risco discreta de cada tratamento assuma a melhor forma. Contudo, se houver interesse em estabelecer comparações, mesmo que empíricas, entre tratamentos, é mais conveniente escolher o mesmo modelo.

O modelo linear pode ser escrito na seguinte forma

$$
\log \left(\frac{h_{i}}{l-h_{i}}\right)=\alpha_{0}+\alpha_{1} t_{i}
$$

onde a matriz modelo e o vetor de parâmetros são dados, respectivamente, por

$$
\boldsymbol{X}^{T}=\left(\begin{array}{cccc}
l & l & \cdots & l \\
t_{1} & t_{2} & \cdots & t_{k}
\end{array}\right),
$$


e

$$
\alpha=\left(\alpha_{0}, \alpha_{1}\right)^{T}
$$

com $k$ intervalos considerados.

O modelo cúbico pode ser escrito na forma

$$
\log \left(\frac{h_{i}}{1-h_{i}}\right)=\alpha_{0}+\alpha_{1} t_{i}+\alpha_{2} t_{i}^{2}+\alpha_{3} t_{i}^{3},
$$

onde a matriz modelo e o vetor de parâmetros são dados, respectivamente, por

$$
X=\left(\begin{array}{cccc}
1 & t_{1} & t_{1}^{2} & t_{1}^{3} \\
1 & t_{2} & t_{2}^{2} & t_{2}^{3} \\
\vdots & \vdots & \vdots & \vdots \\
& & & \\
1 & t_{k} & t_{k}^{2} & t_{k}^{3}
\end{array}\right)
$$

e

$$
\alpha=\left(\alpha_{0}, \alpha_{1}, \alpha_{2}, \alpha_{3}\right)^{T}
$$

Por fim, o modelo cúbico-linear "spline" pode ser escrito como

$$
\log \left(\frac{h_{i}}{I-h_{i}}\right)=x_{i} \alpha=\left(\begin{array}{llll}
1 & t_{i} & \left(t_{i}-11\right)_{-}^{2} & \left(t_{i}-11\right)_{-}^{3}
\end{array}\right)\left(\begin{array}{c}
\alpha_{0} \\
\alpha_{1} \\
\alpha_{2} \\
\alpha_{3}
\end{array}\right)
$$

onde $\left(t_{i}-11\right)=\min \left(0,\left(t_{i}-11\right)\right)$. Assim, a matriz modelo e o vetor de parâmetros são dados, nesse caso, respectivamente, por 


$$
X=\left(\begin{array}{cccc}
I & t_{1} & \left(t_{1}-11\right)^{2} & \left(t_{1}-11\right)^{3} \\
I & t_{2} & \left(t_{2}-11\right)^{2} & \left(t_{2}-11\right)^{3} \\
\vdots & \vdots & \vdots & \vdots \\
& & & \\
1 & t_{i} & \left(t_{i}-11\right)^{2} & \left(t_{i}-11\right)^{3} \\
1 & t_{i+1} & 0 & 0 \\
\vdots & \vdots & \vdots & \vdots \\
1 & t_{k} & 0 & 0
\end{array}\right)
$$

onde $t_{i}<11$ e $t_{i+1} \geq 11$, e

$$
\alpha=\left(\alpha_{0}, \alpha_{1}, \alpha_{2}, \alpha_{3}\right)^{T}
$$

Esse último modelo impõe, portanto, que até 11 meses o logaritmo da chance de morte varie segundo uma função cúbica do tempo e depois de 11 meses varie linearmente com o tempo. O ponto de inflexão, a partir do qual muda a forma da curva que relaciona o tempo com o logit da taxa de risco discreta, pode ser determinado com o auxílio dos conhecimentos do pesquisador e também a partir da soma dos desvios referentes aos dois tratamentos, de acordo com a sugestão de Efron. A idéia básica é ajustar vários modelos com diferentes pontos de inflexão e escolher aquele que minimize a soma dos desvios. Novamente vale ressaltar que se o objetivo do estudo for avaliar o comportamento individual de cada tratamento sem necessariamente estabelecer comparações, pode-se considerar pontos de inflexão diferentes.

No caso do tratamento $\mathrm{B}$, foi adicionado, para todos os modelos propostos, um "offset", com o intuito de compensar a diferença de comprimento dos intervalos.

Como pôde ser visto, a escolha do melhor modelo é uma tarefa bastante complexa envolvendo aspectos de diferentes ordens sendo necessário, portanto, estabelecer regras de 
decisão. Aqui, para cada tratamento, todos os três modelos propostos por Efron foram ajustados. Ao ajustar cada modelo procurou-se, inicialmente, verificar a qualidade de ajuste através da estatística do desvio. Em seguida os modelos com mais parâmetros foram comparados com o mais simples, no caso o linear, através da estatística da diferença dos desvios, que nada mais é do que a estatística da razão de verossimilhança, que permite fazer testes acerca de inclusão ou exclusão de parâmetros. A Tabela 2.2a ilustra os resultados obtidos dos ajustes individuais e das comparações.

Tabela 2.2a: Ajustes dos modelos logísticos para os dois tratamentos segundo a função desvio.

\begin{tabular}{|c|c|c|c|c|c|c|c|}
\hline Tratamento & Modelo & g.l. & Desvio & $\begin{array}{c}\text { Nivel } \\
\text { descritivo }\end{array}$ & $\begin{array}{c}\text { Diferença } \\
\text { dos } \\
\text { desvios }\end{array}$ & $\begin{array}{c}\text { g.l. } \\
\text { (diferença) }\end{array}$ & $\begin{array}{c}\text { Nível } \\
\text { descritivo } \\
\text { (diferença) }\end{array}$ \\
\hline \multirow{3}{*}{ A } & linear & 45 & 59,96 & 0,067 & & & \\
\hline & cúbico & 43 & 49,88 & 0,218 & 10,08 & 2 & 0,006 \\
\hline & cúbico-linear & 43 & 47,73 & 0,286 & 12,23 & 2 & 0,002 \\
\hline \multirow{3}{*}{ B } & linear & 59 & 50,99 & 0,762 & & & \\
\hline & cúbico & 57 & 49,24 & 0,758 & 1,75 & 2 & 0,186 \\
\hline & cúbico-linear & 57 & 34,56 & 0,992 & 16,43 & 2 & $<0,001$ \\
\hline
\end{tabular}

Analisando a Tabela 2.2a, nota-se que todos os modelos parecem se ajustar adequadamente aos dados referentes ao tratamento $\mathrm{B}$, mas quando comparado com o linear, o modelo cúbico-linear "spline" apresentou um melhor ajuste. Com relação ao tratamento A, destaca-se também o modelo cúbico-linear "spline". Para concluir com mais segurança quanto ao melhor modelo foi considerada a estatística de Hosmer e Lemeshow apresentada na Tabela 2.2b. Para efetuar os cálculos foram criados para os tratamentos A e B, respectivamente 9 e 12 grupos segundo descrito na Seção 2.2. 
Tabela 2.2b: Ajustes dos modelos logísticos para os dois tratamentos segundo a estatística de Hosmer e Lemeshow.

\begin{tabular}{|c|c|c|c|c|}
\hline Tratamento & Modelo & g.1. & $\begin{array}{c}\text { Estatistica de Hosmer e } \\
\text { Lemeshow }\end{array}$ & $\begin{array}{c}\text { Nível } \\
\text { descritivo }\end{array}$ \\
\hline \multirow{3}{*}{ A } & linear & 7 & 5,230 & 0,632 \\
\hline & cúbico & 7 & 10,884 & 0,144 \\
\hline & cúbico-linear & 7 & 4,609 & 0,708 \\
\hline \multirow{3}{*}{ B } & linear & 10 & 19,454 & 0,035 \\
\hline & cúbico & 10 & 15,924 & 0,102 \\
\hline & cúbico-linear & 10 & 6,350 & 0,785 \\
\hline
\end{tabular}

Assim como a função desvio, a estatística de Hosmer e Lemeshow também apontou para o modelo cúbico-linear "spline" nos dois tratamentos, sendo esse, portanto, o modelo escolhido. As Tabelas 2.3 e 2.4 contém as estimativas dos parâmetros dos modelos ajustados para cada tratamento.

Tabela 2.3: Estimativas dos parâmetros do modelo cúbico-linear "spline" para o tratamento A.

\begin{tabular}{lccc}
\hline \multicolumn{1}{c}{ Coeficientes } & Estimativa & Desvio padrão & $\begin{array}{c}\text { Valor da } \\
\text { estatística } Z\end{array}$ \\
\hline intercepto & $-2,733$ & 0,605 & $-4,514$ \\
coeficiente linear & $-0,019$ & 0,027 & $-0,705$ \\
coeficiente quadrático & 0,087 & 0,035 & 2,499 \\
coeficiente cúbico & 0,009 & 0,003 & 2,806 \\
\hline
\end{tabular}


Tabela 2.4: Estimativas dos parâmetros do modelo cúbico-linear "spline" para o tratamento B.

\begin{tabular}{lccc}
\hline \multicolumn{1}{c}{ Coeficientes } & Estimativa & Desvio padrão & $\begin{array}{c}\text { Valor da } \\
\text { estatística } Z\end{array}$ \\
\hline intercepto & $-3,497$ & 0,585 & $-5,975$ \\
coeficiente linear & $-0,024$ & 0,021 & $-1,124$ \\
coeficiente quadrático & 0,136 & 0,042 & 3,212 \\
coeficiente cúbico & 0,015 & 0,005 & 3,292 \\
\hline
\end{tabular}

As Tabelas 2.5 e 2.6 contém as estimativas das taxas de risco e função de sobrevivência e seus respectivos desvios-padrão aproximados para alguns dos intervalos segundo o modelo cúbico-linear "spline" para os tratamentos A e B.

Tabela 2.5: Estimativas da taxa de risco e função de sobrevivência e seus respectivos desvios-padrão aproximados para alguns dos intervalos segundo o modelo cúbico-linear "spline" referente ao tratamento A.

\begin{tabular}{cccccc}
\hline Mês & $\begin{array}{c}\text { Taxa de } \\
\text { risco }\end{array}$ & $\begin{array}{c}\text { Desvio } \\
\text { padrão }\end{array}$ & & $\begin{array}{c}\text { Função de } \\
\text { sobrevivência }\end{array}$ & $\begin{array}{c}\text { Desvio } \\
\text { padrão }\end{array}$ \\
\hline 1 & 0,0148 & 0,0110 & & 0,9851 & 0,0110 \\
3 & 0,0867 & 0,0214 & & 0,8605 & 0,0425 \\
5 & 0,1461 & 0,0311 & & 0,6418 & 0,0609 \\
7 & 0,1226 & 0,0247 & & 0,4830 & 0,0663 \\
9 & 0,0755 & 0,0202 & & 0,4029 & 0,0643 \\
11 & 0,0513 & 0,0177 & & 0,3593 & 0,0629 \\
15 & 0,0467 & 0,0137 & & 0,2951 & 0,0622 \\
20 & 0,0426 & 0,0112 & & 0,2354 & 0,0594 \\
25 & 0,0388 & 0,0114 & & 0,1917 & 0,0550 \\
30 & 0,0353 & 0,0132 & & 0,1590 & 0,0508 \\
35 & 0,0321 & 0,0154 & & 0,1342 & 0,0479 \\
\hline
\end{tabular}


Tabela 2.5: Continuação.

\begin{tabular}{cccccc}
\hline Mês & $\begin{array}{c}\text { Taxa de } \\
\text { risco }\end{array}$ & $\begin{array}{c}\text { Desvio } \\
\text { padrão }\end{array}$ & & $\begin{array}{c}\text { Função de } \\
\text { sobrevivência }\end{array}$ & $\begin{array}{c}\text { Desvio } \\
\text { padrão }\end{array}$ \\
\hline 40 & 0,0293 & 0,0175 & & 0,1150 & 0,0465 \\
45 & 0,0266 & 0,0192 & & 0,1000 & 0,0464 \\
47 & 0,0256 & 0,0198 & & 0,0948 & 0,0466 \\
\hline
\end{tabular}

Tabela 2.6: Estimativas das taxas de risco e função de sobrevivência e seus respectivos desvios-padrão aproximados para alguns dos intervalos segundo o modelo cúbico-linear "spline" referente ao tratamento B.

\begin{tabular}{cccccc}
\hline Mês & $\begin{array}{c}\text { Taxa de } \\
\text { risco }\end{array}$ & $\begin{array}{c}\text { Desvio } \\
\text { padrão }\end{array}$ & & $\begin{array}{c}\text { Função de } \\
\text { sobrevivência }\end{array}$ & $\begin{array}{c}\text { Desvio } \\
\text { padrão }\end{array}$ \\
\hline 1 & 0,0040 & 0,0046 & & 0,9945 & 0,0067 \\
3 & 0,0581 & 0,0198 & & 0,8747 & 0,0570 \\
5 & 0,1216 & 0,0296 & & 0,5620 & 0,0835 \\
7 & 0,0867 & 0,0207 & & 0,3602 & 0,0808 \\
9 & 0,0397 & 0,0123 & & 0,2871 & 0,0732 \\
11 & 0,0237 & 0,0093 & & 0,2718 & 0,0709 \\
15 & 0,0210 & 0,0074 & & 0,2489 & 0,0675 \\
20 & 0,0187 & 0,0058 & & 0,2255 & 0,0640 \\
31 & 0,0146 & 0,0048 & & 0,1940 & 0,0587 \\
41 & 0,0115 & 0,0053 & & 0,1820 & 0,0563 \\
51 & 0,0091 & 0,0057 & & 0,1730 & 0,0544 \\
61 & 0,0072 & 0,0059 & & 0,1663 & 0,0531 \\
71 & 0,0057 & 0,0058 & & 0,1612 & 0,0525 \\
77 & 0,0049 & 0,0056 & & 0,1587 & 0,0524 \\
\hline
\end{tabular}

Note que para os pacientes submetidos ao tratamento A, radioterapia, o risco de morte por câncer na cabeça e pescoço cresce no início do intervalo estudado, até aproximadamente o 
quinto mês, onde assume o maior valor, 0,1461, e em seguida decresce rapidamente até o décimo primeiro mês, para enfim seguir linearmente até o término do seguimento.

Da mesma forma, para os pacientes submetidos ao tratamento $\mathrm{B}$, radioterapia associada a quimioterapia, o risco de morte por câncer na cabeça e pescoço também cresce no início do intervalo estudado, mas é sempre menor que no tratamento $\mathrm{A}$, como mostram as Tabelas 2.5 e 2.6. Após o crescimento, que também acontece até aproximadamente o quinto mês quando assume o maior valor, 0,1216, a taxa de risco decresce rapidamente até o décimo primeiro mês, para depois descrescer linearmente até o término do seguimento.

Com intuito de estabelecer uma relação quantitativa entre as chances de morte no dois tratamentos, considerou-se uma medida equivalente à razão das chances. Constatou-se que na ocasião do quinto mês, onde o risco de morte é maior no dois tratamentos, a chance de morte no tratamento A é 1,863 vezes maior do que no tratamento B e após 20 meses de seguimento a chance de morte no tratamento A já é 2,373 vezes maior do que no tratamento B. Vale ressaltar que essa medida é empírica tendo como propósito dar uma idéia comparativa dos dois tratamentos.

Para efeito de vizualização, foram feitos gráficos contendo as curvas ajustadas de cada tratamento tanto para a taxa de risco discreta quanto para a função de sobrevivência. Através dos Gráficos 2.1 e 2.2, nota-se que no início do período estudado a taxa de risco discreta nos tratamentos A e B são próximas, e consequentemente, as curvas de sobrevida se confundem. Entretanto após o período de alto risco, ou seja, após 11 meses, o risco de morte por câncer na cabeça e pescoço parace ser maior no tratamento $\mathrm{A}$, ou seja, entre os pacientes submetidos à radioterapia isolada.

Os Gráficos 2.3 e 2.4 mostram as curvas referentes às taxas de risco e as bandas com $95 \%$ de confiança em cada tratamento separadamente. Analogamente, os Gráficos 2.5 e 2.6 mostram as curvas de sobrevida e as bandas com $95 \%$ de confiança também para cada tratamento. 
Para cada um dos modelos ajustados, foi feita uma análise de resíduos. Os Gráficos 2.7, 2.8 e 2.9 são referentes ao tratamento A e os Gráficos $2.10,2.11$ e 2.12 são referentes ao tratamento B.

Com relação ao tratamento A, o Gráfico 2.7 do componente do desvio padronizado $t_{D_{i}}$ contra os valores ajustados das taxas de risco, indica a presença de alguns pontos fora dos limites toleráveis, sendo, portanto, pontos aberrantes. O Gráfico 2.8 da distância de Cook, $L D_{i}$, pelos valores ajustados das probabilidades, indica que, o ponto referente ao intervalo 4 é um ponto influente pois, quando presente nos dados, altera em mais de $20 \%$ as estimativas dos coeficientes do modelo ajustado. Essa influência pode ser explicada pelo fato de apresentar um número menor de eventos do que o esperado, segundo a tendência apresentada nos intervalos anteriores, como pode ser visto no Apêndice A. O Gráfico 2.9 dos resíduos padronizados e envelopes, indica que todos os pontos encontram-se dentro das bandas, entretanto, existem indícios de superdispersão, pois resíduos negativos estão mais próximos da banda inferior e resíduos positivos estão mais próximos da banda superior, sugerindo, então, a correção por um parâmetro de dispersão.

Com relação ao tratamento B, o Gráfico 2.10 do componente do desvio padronizado $t_{D_{i}}$ contra os valores ajustados das taxas de risco, indica que todos os pontos encontram-se dentro dos limites toleráveis e apresentam a tendência esperada de acordo com a Tabela 2.1. O Gráfico 2.11 da distância de Cook, $L D_{i}$, pelos valores ajustados das probabilidades, indica que não existem pontos influentes. A partir do Gráfico 2.12, que contém os resíduos padronizados e envelopes, nota-se que todos os pontos encontram-se dentro das bandas e não existem indícios de superdispersão, e portanto, não há evidências de que o modelo esteja mal ajustado ou que as suposições feitas não sejam válidas. 
Gráfico 2.1: Taxas de risco estimada para cada tratamento sob modelo cúbico-linear.

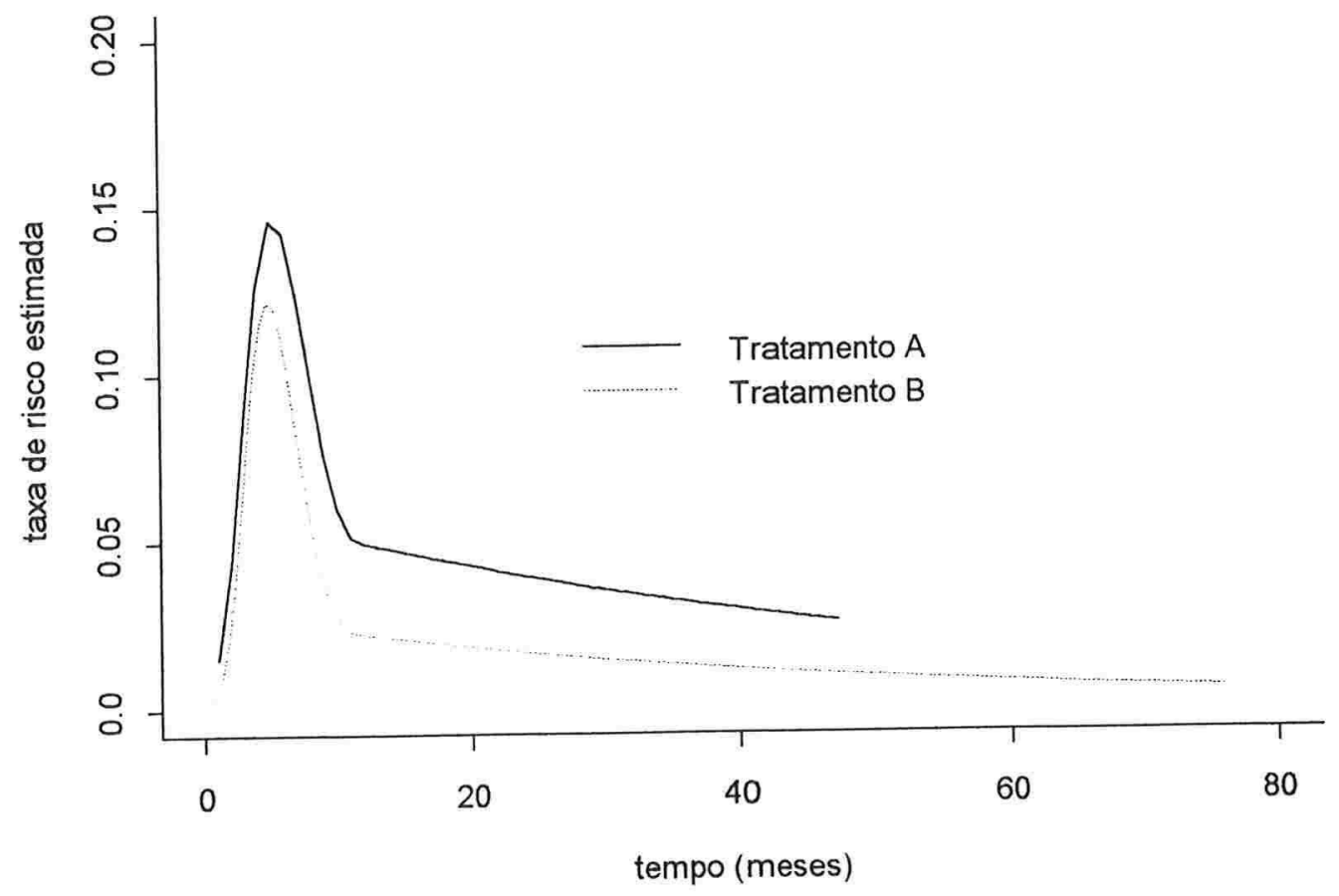

Gráfico 2.2:Curva de sobrevida estimada para cada tratamento sob modelo cúbico-linear.

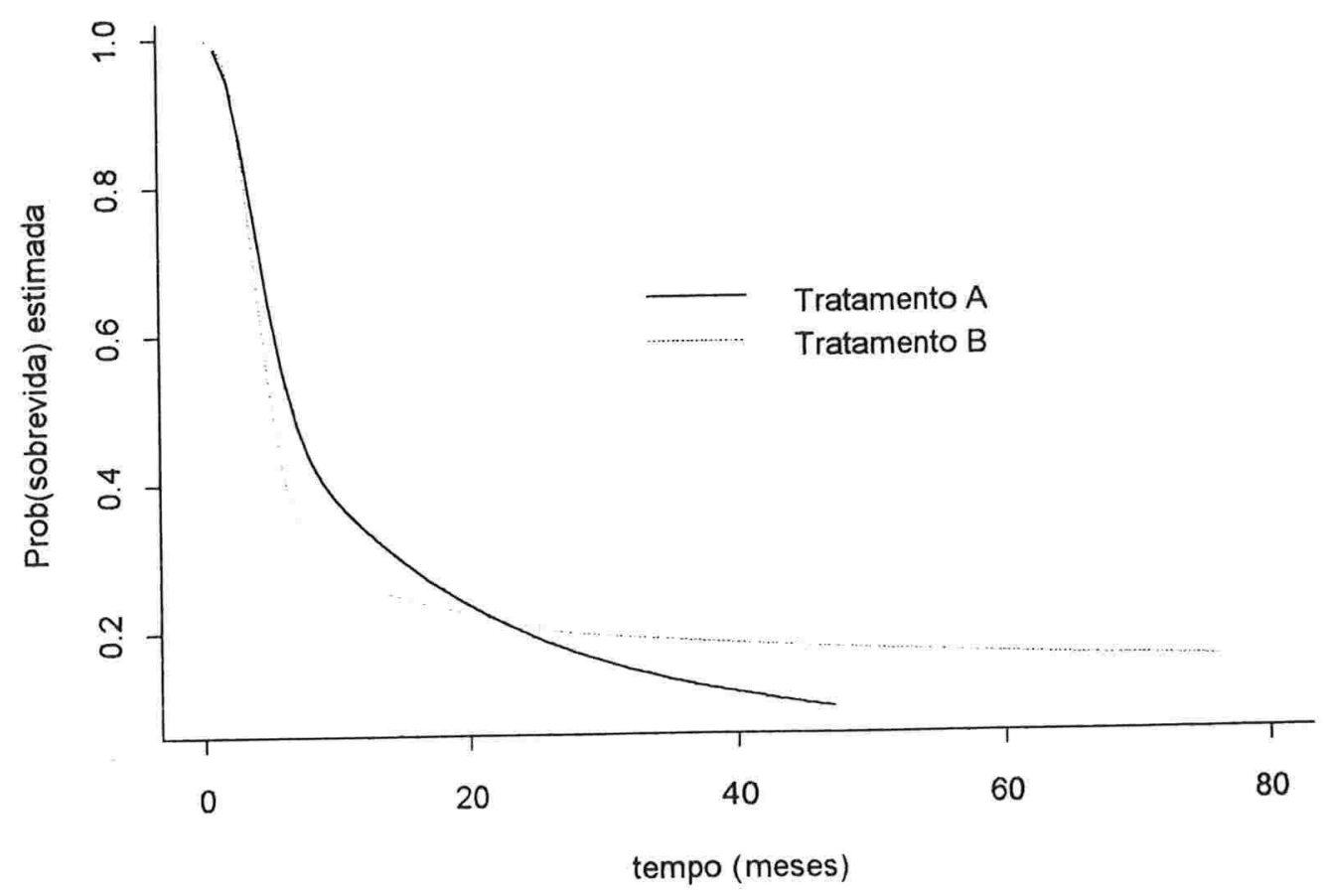


Gráfico 2.3: Taxa de risco e bandas de confiança (95\%) estimadas para o tratamento A.

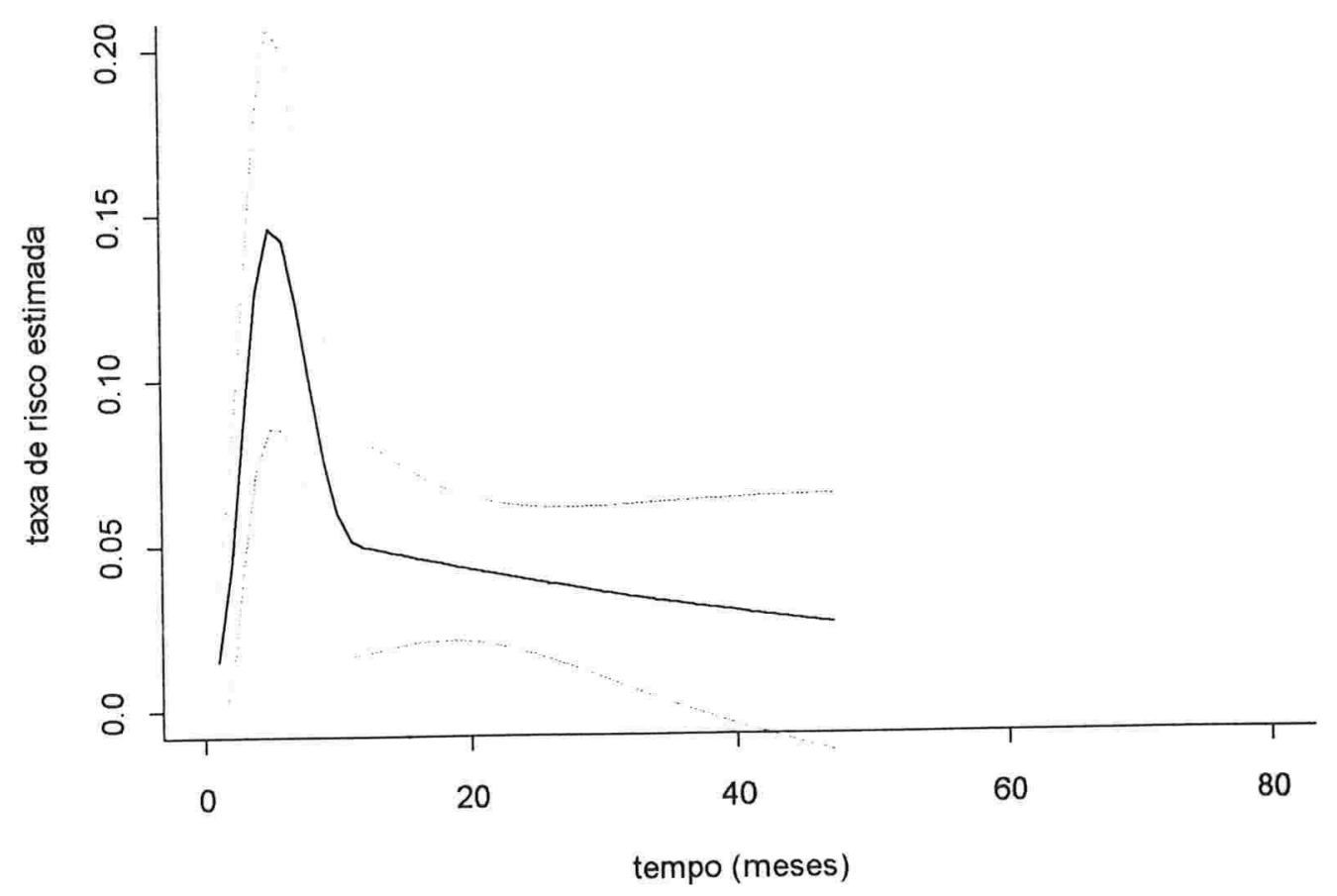

Gráfico 2.4: Taxa de risco e bandas de confiança (95\%) estimadas para o tratamento B.

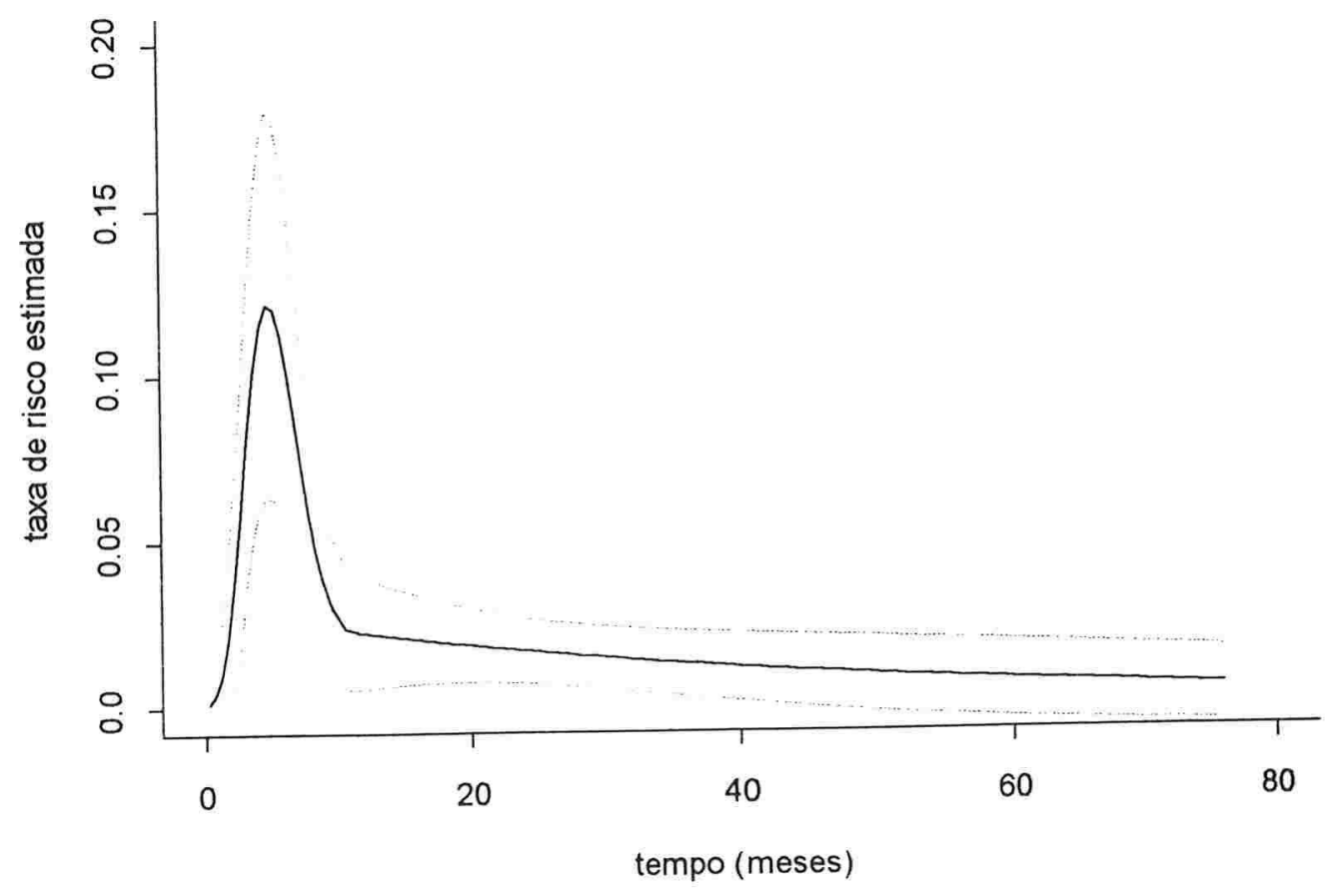


Gráfico 2.5: Curva de sobrevida e bandas de confiança (95\%) estimadas para o tratamento A.

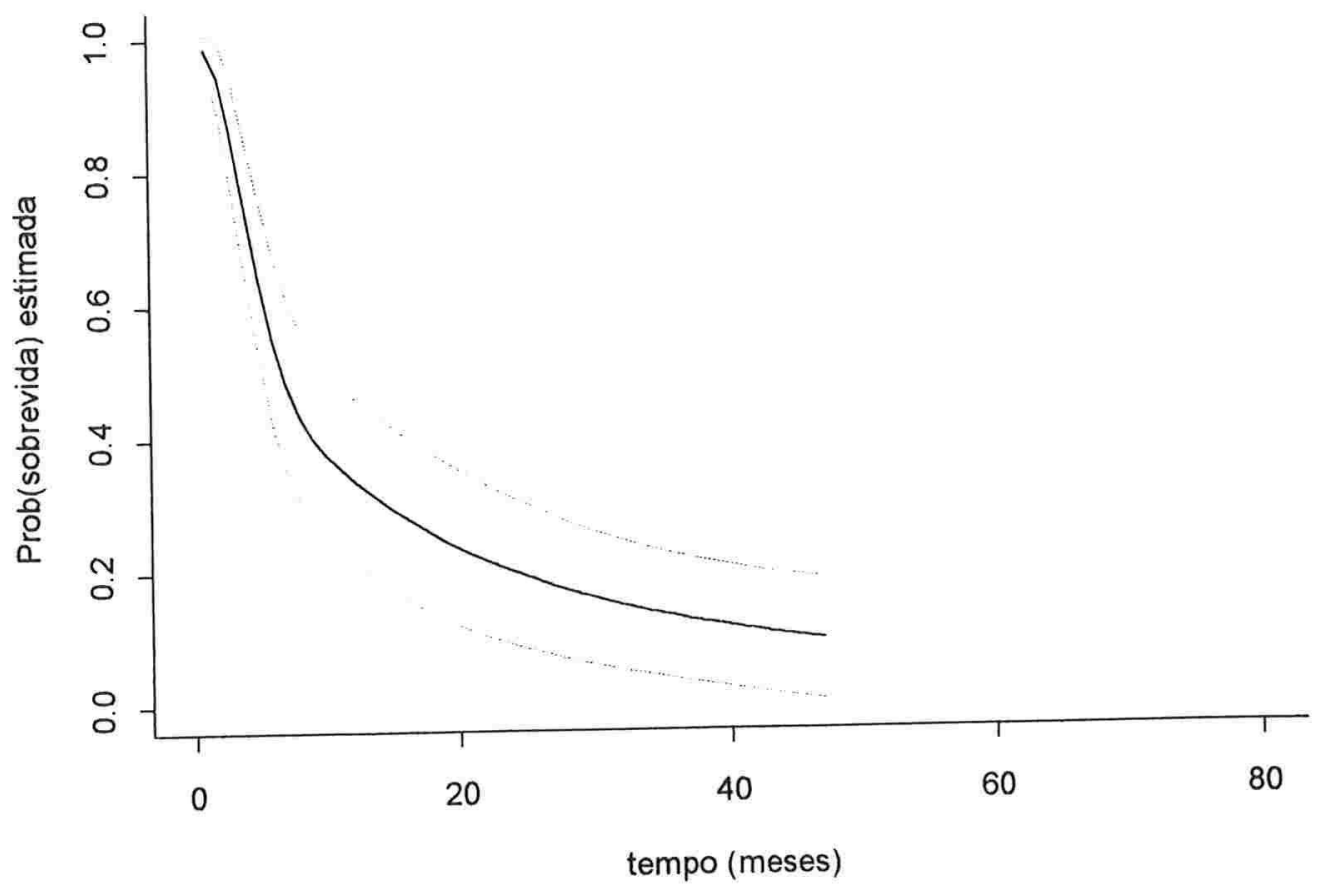

Gráfico 2.6: Curva de sobrevida e bandas de confiança (95\%) estimadas para o tratamento B.

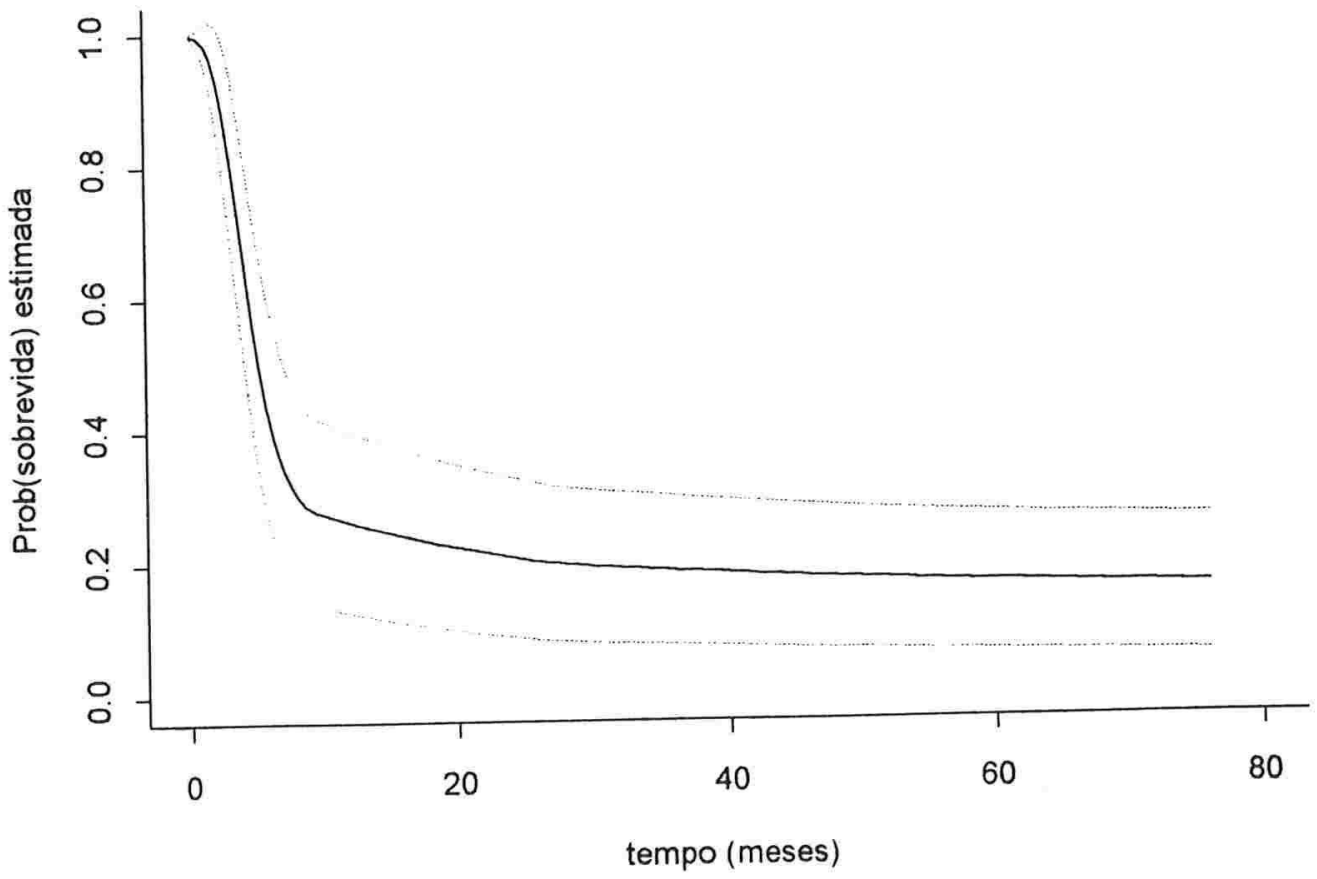


Gráfico 2.7: Gráfico do $t_{D_{i}}$ para detectar pontos aberrantes no tratamento $\mathrm{A}$.

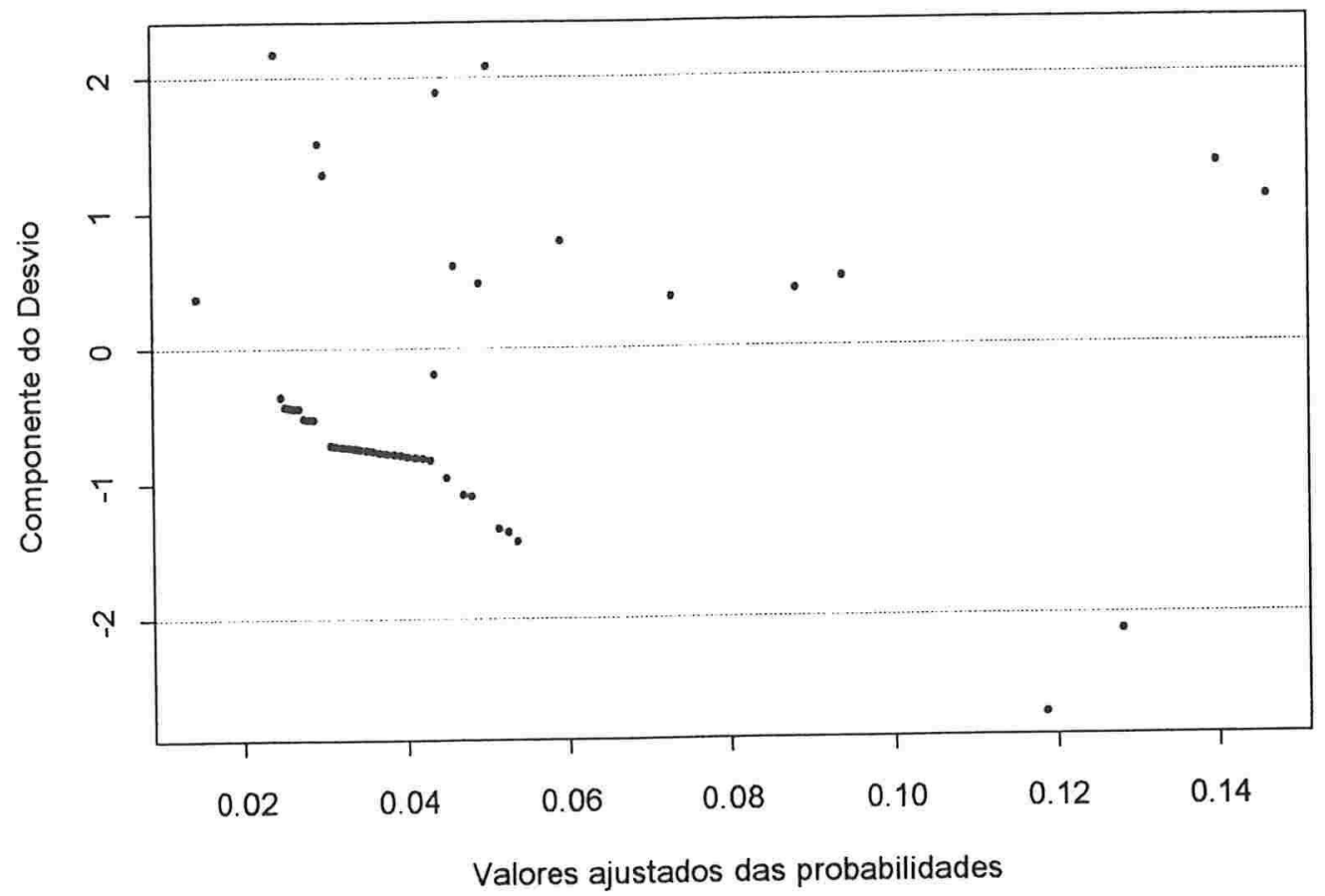

Gráfico 2.8: Gráfico do $L D_{i}$ para detectar pontos influentes no tratamento A.

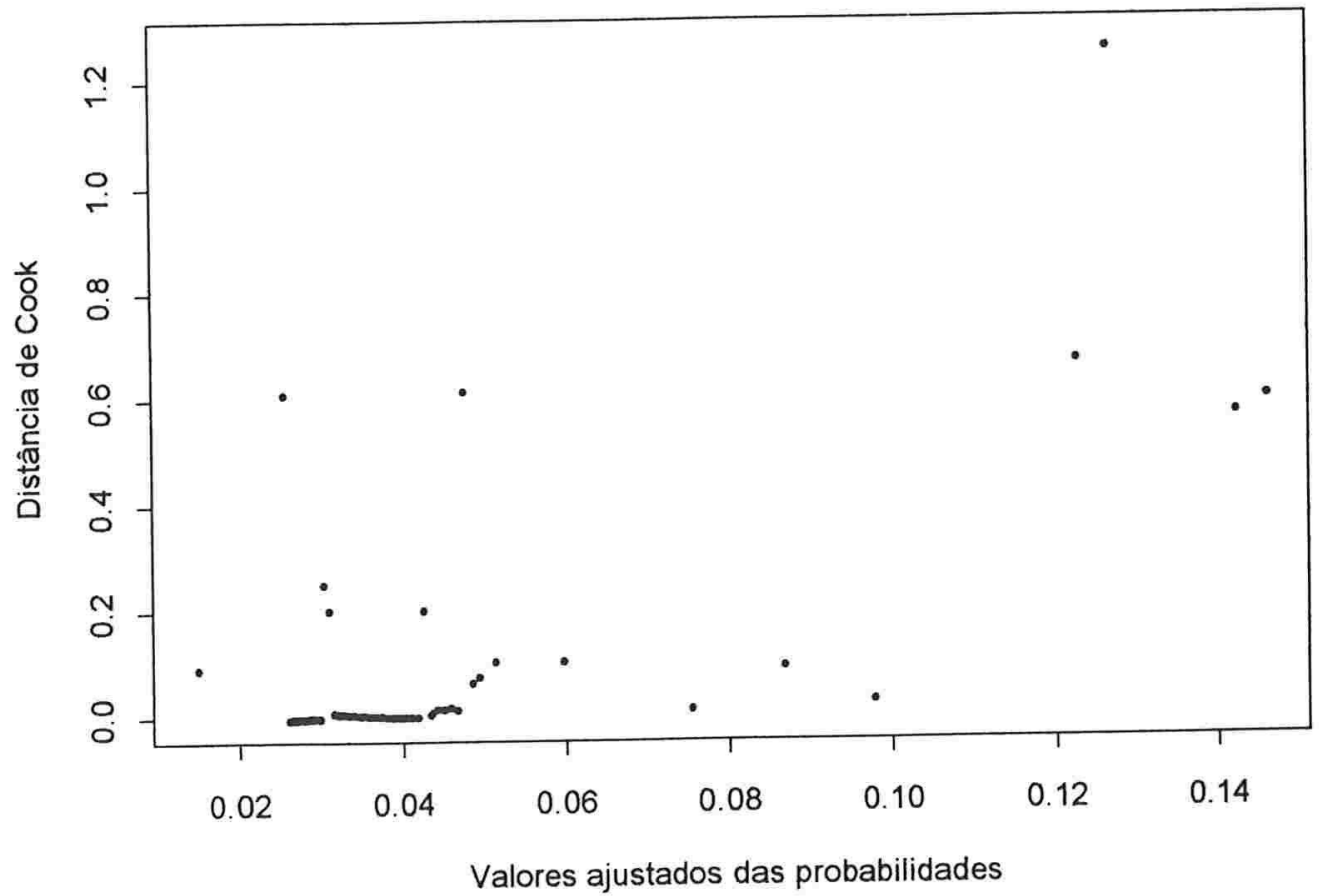


Gráfico 2.9: Resíduos padronizados e envelopes para o tratamento A.

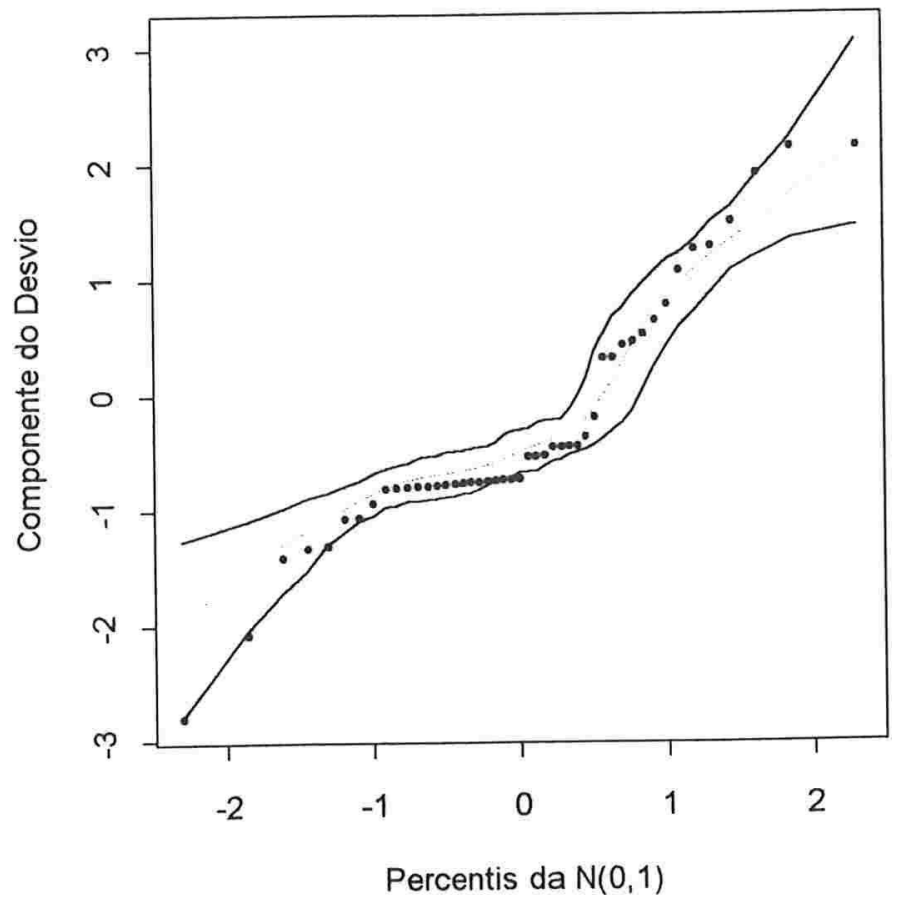

Gráfico 2.10: Gráfico do $t_{D_{i}}$ para detectar pontos aberrantes no tratamento B.

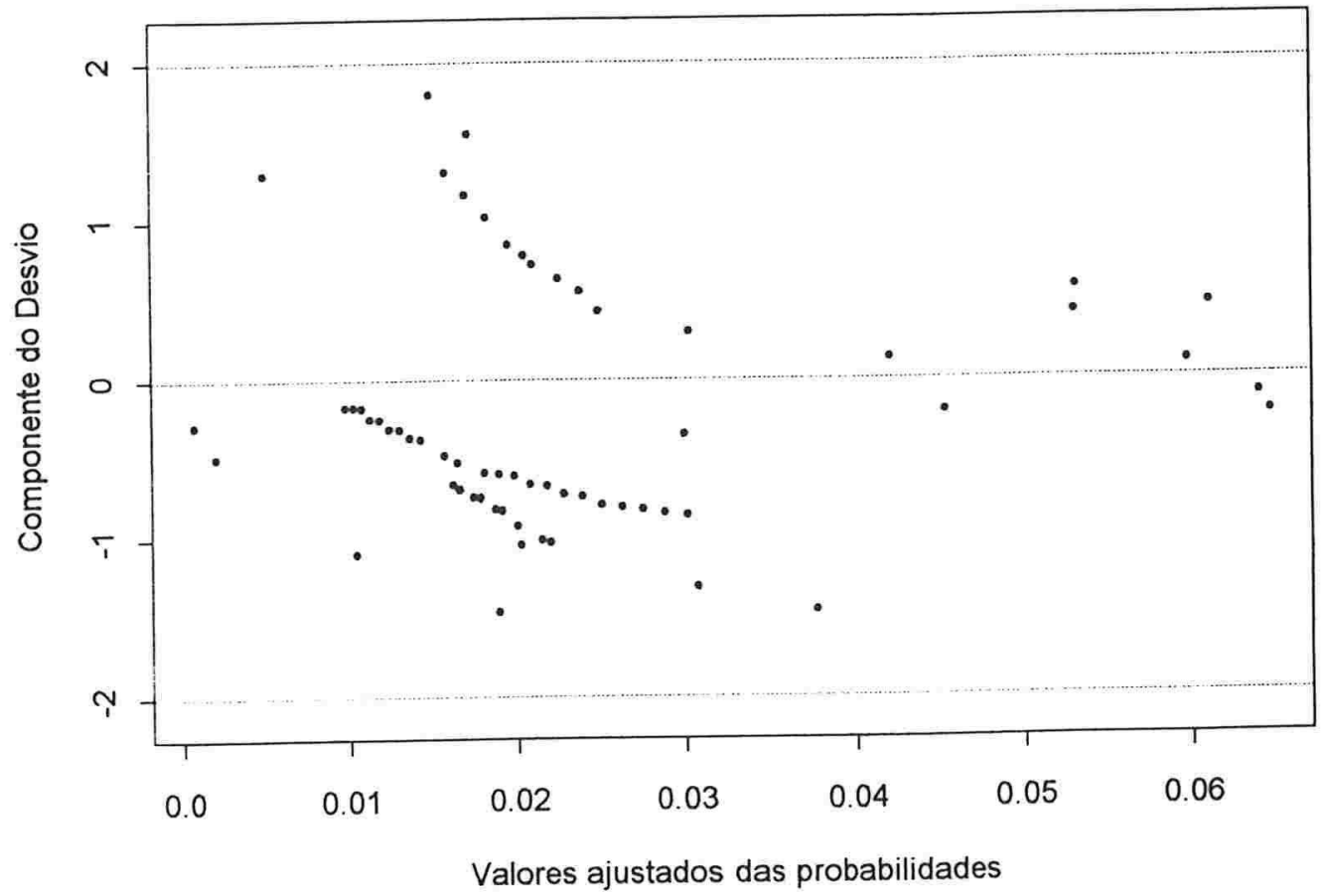


Gráfico 2.11: Gráfico do $L D_{i}$ para detectar pontos influentes no tratamento B.

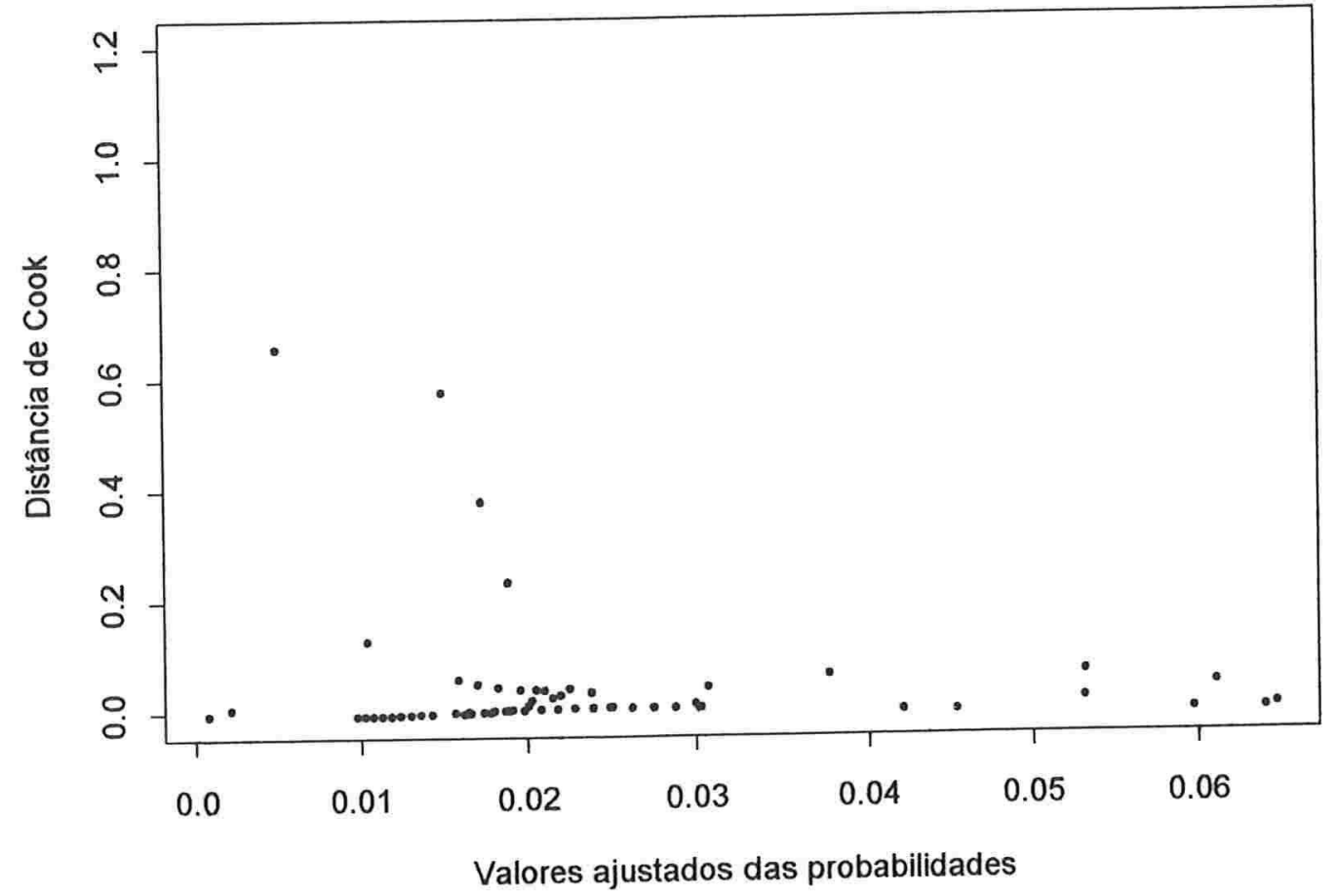

Gráfico 2.12: Resíduos padronizados e envelopes para o tratamento B.

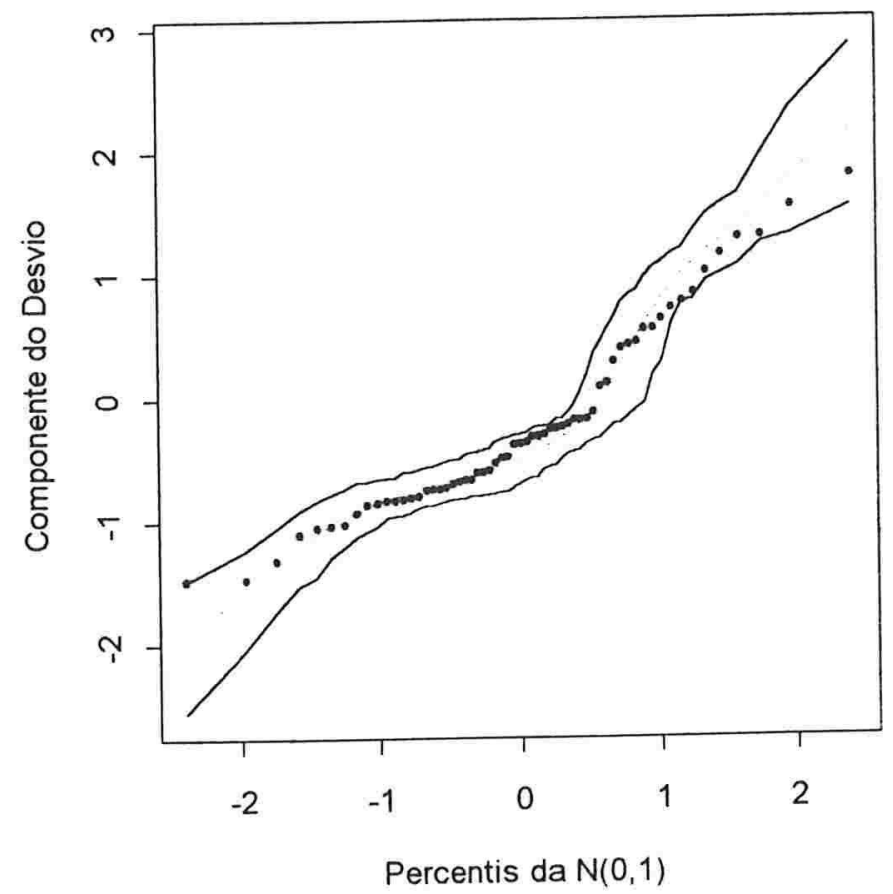


O problema da superdispersão detectada no tratamento A pode ser contornado utilizando um modelo de quasi-verossimilhança, de tal forma que o parâmetro de dispersão possa ser estimado. Considerando esse modelo com a mesma função de variância do modelo binomial e mesma função de ligação entre a média e o preditor linear, ou seja, o logit, obtemos as mesmas estimativas pontuais para os parâmetros do modelo, entretanto as estimativas dos desvios-padrão e resíduos sofreram um aumento em função do parâmetro de dispersão estimado por 1,348 .

Tabela 2.7: Estimativas dos parâmetros do modelo de quasiverossimilhança cúbico-linear "spline" para o tratamento A.

\begin{tabular}{lccc}
\hline \multicolumn{1}{c}{ Coeficientes } & Estimativa & Desvio padrão & $\begin{array}{c}\text { Valor da } \\
\text { estatística } Z\end{array}$ \\
\hline intercepto & $-2,733$ & 0,712 & $-3,838$ \\
coeficiente linear & $-0,019$ & 0,032 & $-0,598$ \\
coeficiente quadrático & 0,087 & 0,041 & 2,125 \\
coeficiente cúbico & 0,009 & 0,004 & 2,386 \\
\hline
\end{tabular}

Naturalmente as estimativas da taxa de risco e função de sobrevivência não sofrem nenhuma alteração, entretanto, os seus desvios-padrão aumentam, uma vez que dependem da matriz de covariância do vetor de parâmetros, que foi inflacionada pelo parâmetro de dispersão. Essa alteração está apresentada na Tabela 2.8. 
Tabela 2.8: Estimativas da taxa de risco e função de sobrevivência e seus respectivos desvios-padrão aproximados para alguns dos intervalos no modelo de quasi-verossimilhança cúbico-linear "spline" referente ao tratamento A.

\begin{tabular}{cccccc}
\hline Mês & $\begin{array}{c}\text { Taxa de } \\
\text { risco }\end{array}$ & $\begin{array}{c}\text { Desvio } \\
\text { padrão }\end{array}$ & & $\begin{array}{c}\text { Função de } \\
\text { sobrevivência }\end{array}$ & $\begin{array}{c}\text { Desvio } \\
\text { padrão }\end{array}$ \\
\cline { 1 - 3 } \cline { 5 - 6 } 1 & 0,0148 & 0,0130 & & 0,9851 & 0,0129 \\
3 & 0,0867 & 0,0252 & & 0,8605 & 0,0500 \\
5 & 0,1461 & 0,0365 & & 0,6418 & 0,0717 \\
7 & 0,1226 & 0,0291 & & 0,4830 & 0,0780 \\
9 & 0,0755 & 0,0237 & & 0,4029 & 0,0756 \\
11 & 0,0513 & 0,0208 & & 0,3593 & 0,0740 \\
15 & 0,0467 & 0,0161 & & 0,2951 & 0,0732 \\
20 & 0,0426 & 0,0131 & & 0,2354 & 0,0699 \\
25 & 0,0388 & 0,0134 & & 0,1917 & 0,0647 \\
30 & 0,0353 & 0,0156 & & 0,1590 & 0,0598 \\
35 & 0,0321 & 0,0182 & & 0,1342 & 0,0563 \\
40 & 0,0293 & 0,0206 & & 0,1150 & 0,0547 \\
45 & 0,0266 & 0,0226 & & 0,1000 & 0,0546 \\
47 & 0,0256 & 0,0233 & 0,0948 & 0,0549 \\
\hline
\end{tabular}

O Gráfico 2.13 do resíduos padronizados corrigidos pelo parâmetro de dispersão com envelopes indica uma melhora no ajuste do modelo, já que os resíduos estão mais centralizados dentro das bandas. 
Gráfico 2.13: Resíduos padronizados e envelopes para o tratamento A no modelo de quasiverossimilhança.

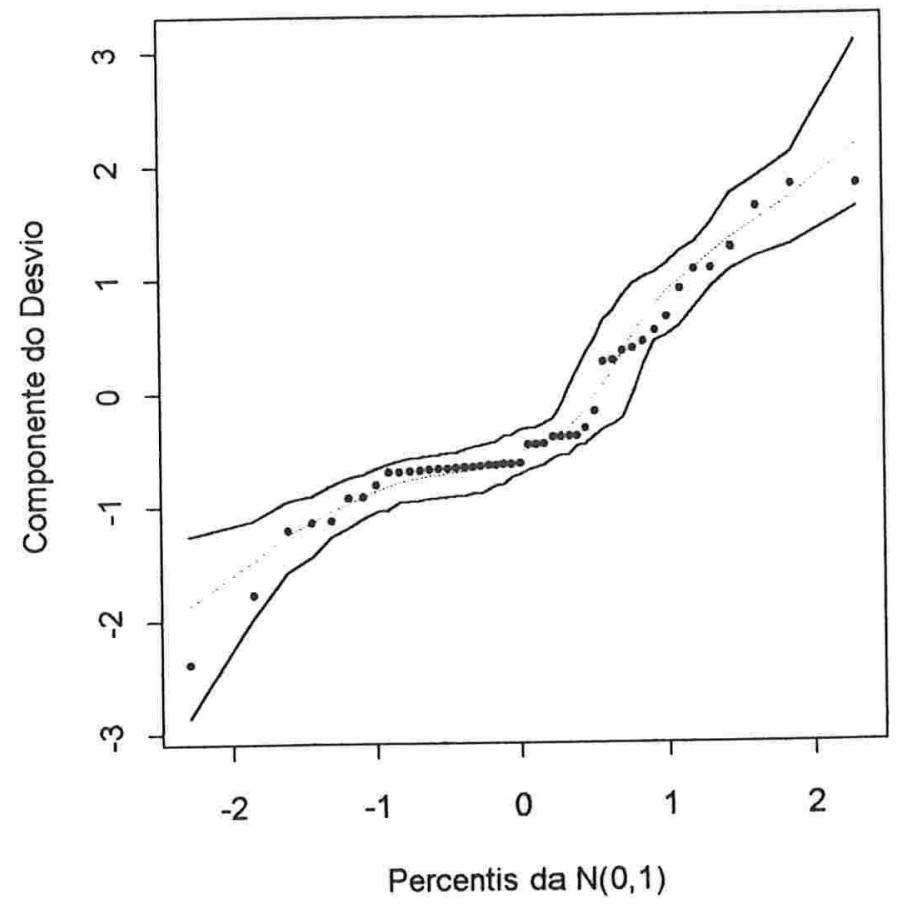




\section{CAPÍtulu 3}

\section{APLICAÇÕES}

Neste capítulo serão apresentados três exemplos de aplicação da regressão logística parcial, um deles na área de educação e dois da área biomédica. É importante ressaltar que, apesar dessa técnica ser aplicável em qualquer conjunto de dados em que se observa o tempo até a ocorrência de algum evento, é mais comum encontrar exemplos na área biomédica.

\subsection{Estudo sobre a carreira de educadores de crianças excepcionais}

Procurou-se estudar a carreira de 3941 educadores de crianças excepcionais contratados em uma escola pública do estado de Michigan (EUA) entre os anos de 1972 e 1978 (Singer e Willett, 1995). Até o ano de 1985, quando o estudo foi concluído, observou-se o tempo entre a contratação dos professores e o abandono da carreira. Portanto, nesse estudo o termo "ano" não se refere ao ano cronológico, mas ao tempo de serviço naquela escola. Em 1985, 2207 educadores, ou seja, 56\% da amostra total haviam abandonado a carreira e 1734 foram censurados. O dados censurados correspondem, nesse caso, a educadores que foram 
promovidos a outros cargos dentro da mesma escola. A Tabela 3.1.1 contém os dados em questão.

Tabela 3.1.1: Dados sobre a carreira de educadores de crianças excepcionais da escola pública de Michigan.

\begin{tabular}{cccc}
\hline Ano & $\begin{array}{c}\text { oo de professores } \\
\text { lecionando no } \\
\text { início do ano }\end{array}$ & $\begin{array}{c}\mathrm{N}^{\mathbf{0}} \text { de professores que } \\
\text { largaram a carreira } \\
\text { durante o ano }\end{array}$ & $\begin{array}{c}\mathrm{N}^{\circ} \text { de professores } \\
\text { censurado no } \\
\text { final do ano }\end{array}$ \\
\hline 1 & 3941 & 456 & 0 \\
2 & 3485 & 384 & 0 \\
3 & 3101 & 359 & 0 \\
4 & 2742 & 295 & 0 \\
5 & 2447 & 218 & 0 \\
6 & 2229 & 184 & 0 \\
7 & 2045 & 123 & 280 \\
8 & 1642 & 79 & 307 \\
9 & 1256 & 53 & 255 \\
10 & 948 & 35 & 265 \\
11 & 648 & 16 & 241 \\
12 & 391 & 5 & 386 \\
\hline
\end{tabular}

Com o intuito de modelar o risco de largar a carreira ao longo dos anos de serviço foi aplicada a técnica de regressão logística parcial. Inicialmente, procurou-se estudar a relação da taxa de risco observada com o tempo de serviço através do Gráfico 3.1.1, de onde se conclui que o risco de abandonar a carreira diminui a medida que aumenta o tempo de serviço.

Como trata-se de uma função aparentemente simples, procurou-se ajustar somente modelos simples como o linear, quadrático e cúbico. Os modelos quadrático e cúbico foram comparados com o linear através da estatística da razão de verossimilhança. A Tabela 3.1.2a contém as estatísticas do desvio para cada modelo e as estatísticas que comparam os modelos com mais parâmetros com o linear. A Tabela 3.1.2b contém as estatísticas de ajuste de Hosmer e Lemeshow para cada modelo, que foram calculadas considerando-se 12 grupos com um único elemento. 
Tabela 3.1.2a: Ajustes dos modelos logísticos segundo a função desvio.

\begin{tabular}{lrccccc}
\hline Modelo & g.1. & Desvio & $\begin{array}{c}\text { Nivel } \\
\text { descritivo }\end{array}$ & $\begin{array}{c}\text { Diferença } \\
\text { dos } \\
\text { desvios }\end{array}$ & $\begin{array}{c}\text { g.l. } \\
\text { (diferença) }\end{array}$ & $\begin{array}{c}\text { Nível } \\
\text { descritivo } \\
\text { (diferença) }\end{array}$ \\
\hline linear & 10 & 43,287 & 0,000 & & & \\
quadrático & 9 & 6,775 & 0,661 & 36,512 & 1 & 0,000 \\
cúbico & 8 & 5,764 & 0,674 & 37,523 & 2 & 0,000 \\
\hline
\end{tabular}

Tabela 3.1.2b: Ajustes dos modelos logísticos segundo a estatística de Hosmer e Lemeshow.

\begin{tabular}{lccc}
\hline Modelo & g.1. & $\begin{array}{c}\text { Estatística de Hosmer e } \\
\text { Lemeshow }\end{array}$ & $\begin{array}{c}\text { Nível } \\
\text { descritivo }\end{array}$ \\
\hline linear & 10 & 41,823 & 0,000 \\
quadrático & 10 & 5,723 & 0,838 \\
cúbico & 10 & 6,803 & 0,744 \\
\hline
\end{tabular}

Ao contrário do modelo linear, os modelos quadrático e cúbico apresentaram um bom ajuste, sendo portanto, mais adequados. Nesse caso, foi adotado o modelo quadrático por possuir menos parâmetros a serem estimados. A Tabela 3.1.3 contém as estimativas dos parâmetros do modelo ajustado e a Tabela 3.1 .4 e contém as estimativas da taxa de risco e função de sobrevivência e seus respectivos desvios-padrão aproximados.

Tabela 3.1.3: Estimativas dos parâmetros do modelo quadrático.

\begin{tabular}{lccc}
\hline \multicolumn{1}{c}{ Coeficientes } & Estimativa & $\begin{array}{c}\text { Desvio } \\
\text { padrão }\end{array}$ & $\begin{array}{c}\text { Valor da } \\
\text { estatistica } Z\end{array}$ \\
\hline intercepto & $-2,043$ & 0,053 & $-38,811$ \\
coeficiente linear & 0,030 & 0,029 & 1,020 \\
coeficiente quadrático & $-0,019$ & 0,003 & $-5,812$ \\
\hline
\end{tabular}

Analisando a Tabela 3.1.4 e os Gráficos 3.1 .2 e 3.1 .3 que contém, respectivamente, a curva ajustada da taxa de risco com bandas de $95 \%$ de confiança e da função de sobrevivência 
também com bandas de $95 \%$ de confiança, nota-se que o risco de abandonar a carreira é maior no início do período diminuindo a medida que aumenta o tempo de serviço. Consequentemente, a função de sobrevida, que nesse caso representa o tempo até abandonar a carreira, decai mais rapidamente no início apresentando uma tendência a se estabilizar no final do período.

Tabela 3.1.4: Estimativas da taxa de risco e função de sobrevivência e seus respectivos desvios-padrão aproximados no modelo quadrático.

\begin{tabular}{|c|c|c|c|c|}
\hline Anos & $\begin{array}{c}\text { Taxa de } \\
\text { risco }\end{array}$ & $\begin{array}{l}\text { Desvio } \\
\text { padrão }\end{array}$ & $\begin{array}{c}\text { Função de } \\
\text { sobrevivência }\end{array}$ & $\begin{array}{l}\text { Desvio } \\
\text { padrão }\end{array}$ \\
\hline 1 & 0,1158 & 0,0042 & 0,8842 & 0,0042 \\
\hline 2 & 0,1149 & 0,0028 & 0,7826 & 0,0060 \\
\hline 3 & 0,1104 & 0,0026 & 0,6961 & 0,0067 \\
\hline 4 & 0,1025 & 0,0028 & 0,6247 & 0,0071 \\
\hline 5 & 0,0920 & 0,0028 & 0,5673 & 0,0074 \\
\hline 6 & 0,0796 & 0,0026 & 0,5221 & 0,0076 \\
\hline 7 & 0,0664 & 0,0023 & 0,4875 & 0,0077 \\
\hline 8 & 0,0533 & 0,0023 & 0,4615 & 0,0078 \\
\hline 9 & 0,0412 & 0,0025 & 0,4425 & 0,0079 \\
\hline 10 & 0,0306 & 0,0026 & 0,4290 & 0,0080 \\
\hline 11 & 0,0218 & 0,0026 & 0,4196 & 0,0083 \\
\hline 12 & 0,0150 & 0,0024 & 0,4133 & 0,0086 \\
\hline
\end{tabular}

Com intuito de detectar a presença de pontos aberrantes e influentes e verificar se as suposições do modelo estão sendo satisfeitas, foi feita uma análise de resíduos. O Gráfico 3.1.4 do componente do desvio padronizado contra os valores ajustados da taxa de risco, indica que todos os resíduos estão dentro dos limites toleráveis. Pelo Gráfico 3.1.5 da distância de Cook, pelos valores ajustados das probabilidades, conclui-se que não há pontos influentes. Por fim, através do Gráfico 3.1.6, que contém os resíduos padronizados e envelopes, nota-se que todos os pontos encontram-se dentro das bandas de confiança não apresentando nenhuma tendência o que indica que o modelo está bem ajustado e que as suposições são válidas. 
Gráfico 3.1.1: Taxas de risco observada.

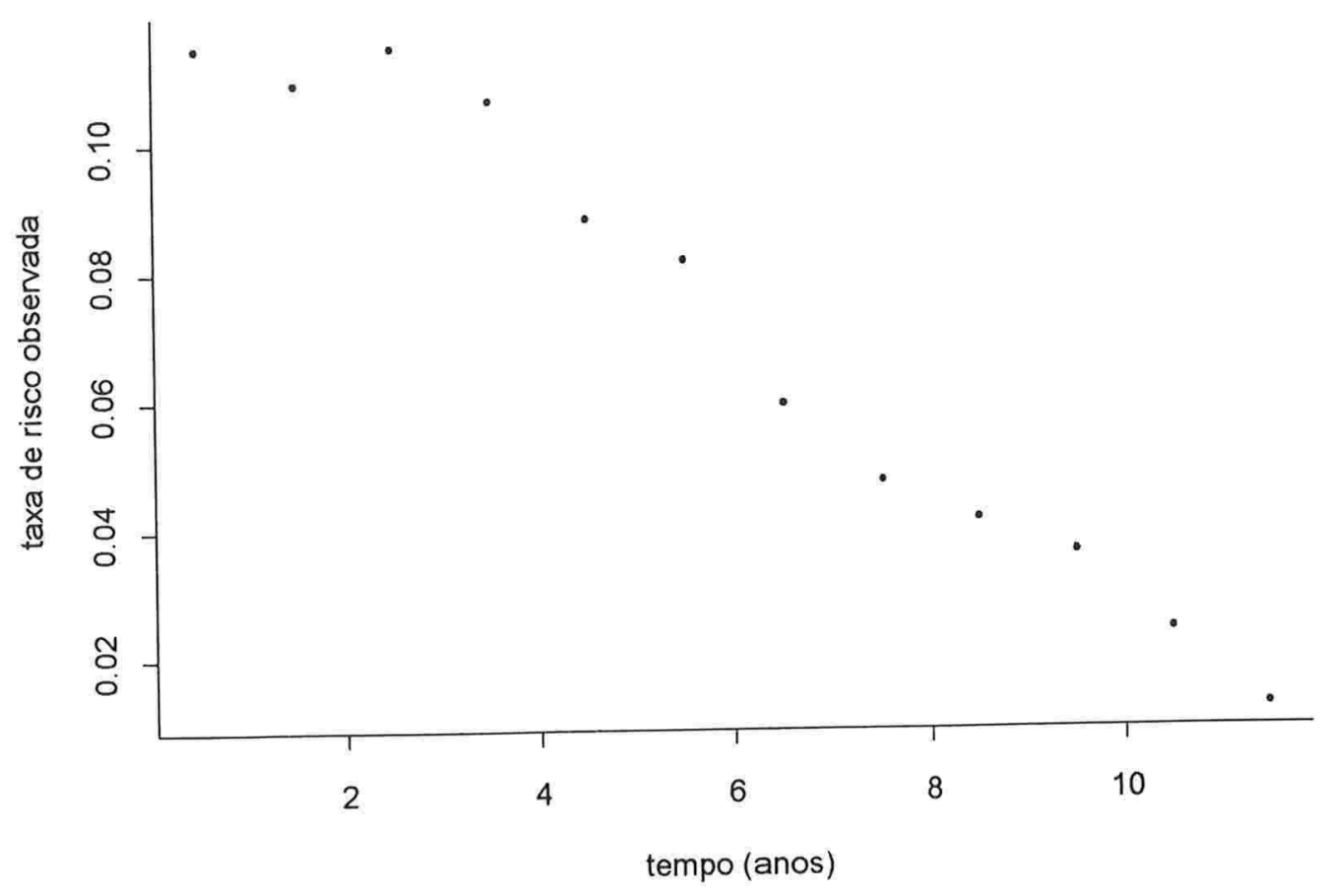

Gráfico 3.1.2: Taxa de risco estimada e bandas de confiança (95\%) para o modelo quadrático.

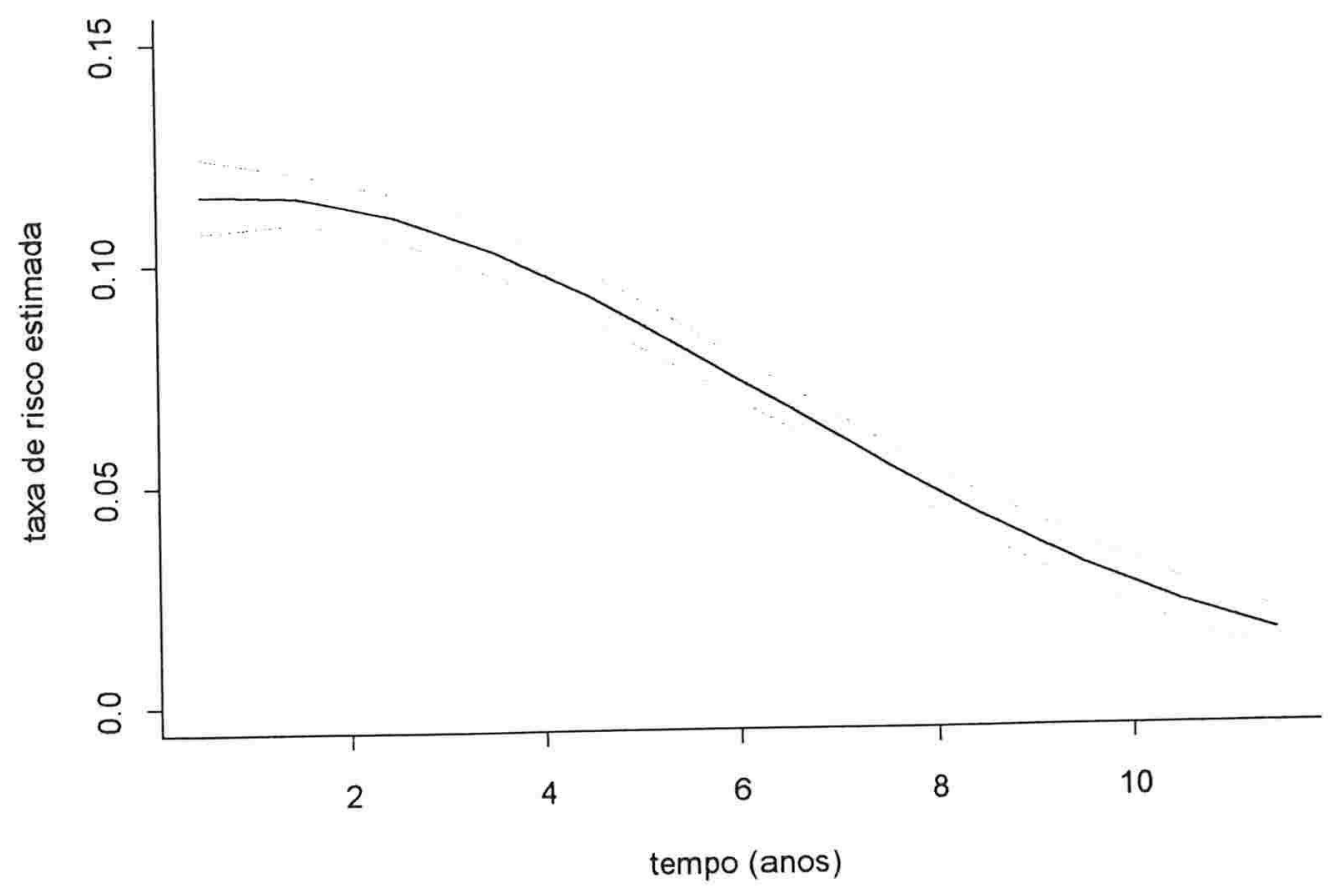


Gráfico 3.1.3: Curva de sobrevida e bandas de confiança (95\%) estimadas para o modelo quadrático.

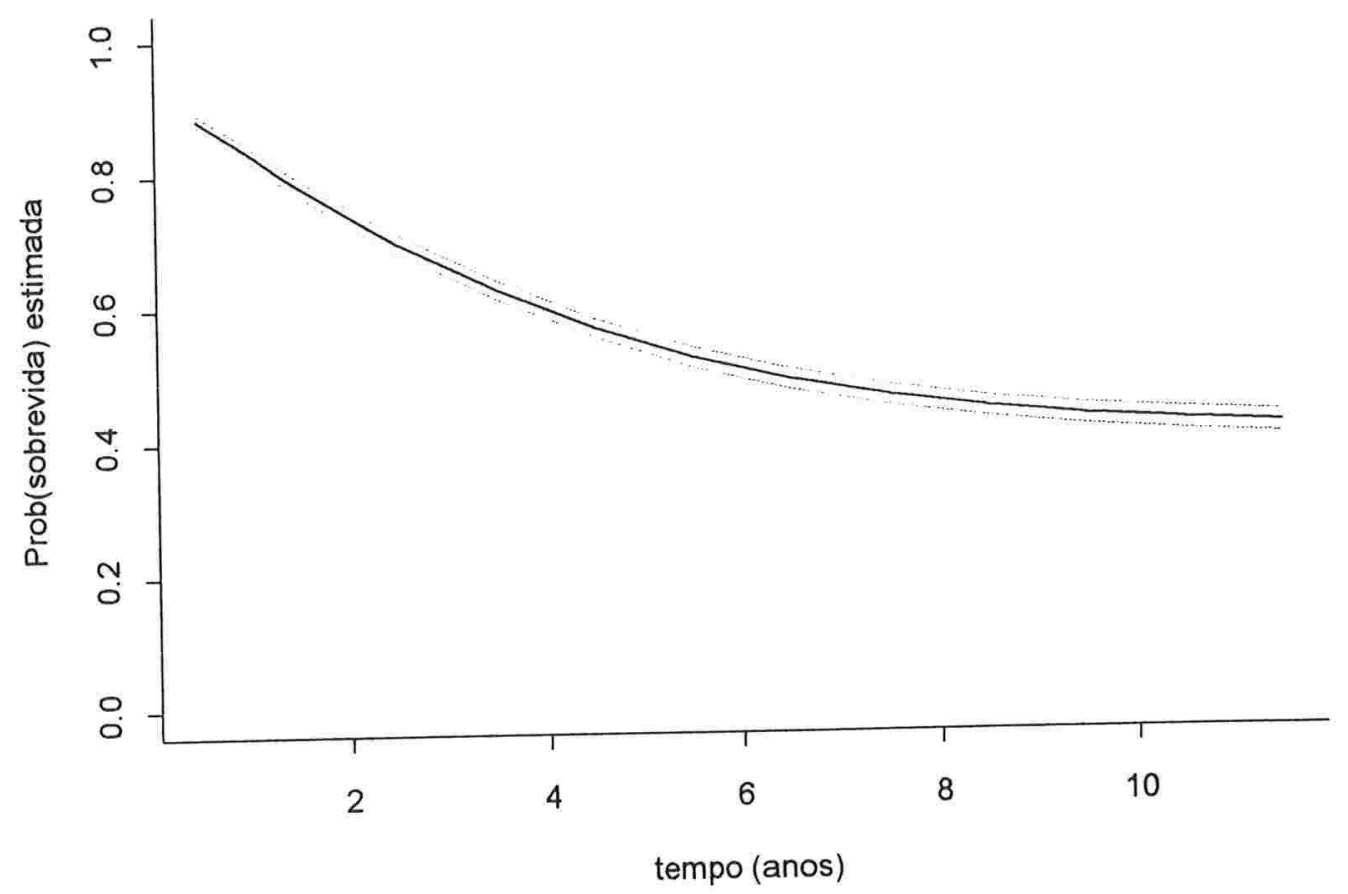

Gráfico 3.1.4: Gráfico do $t_{D_{i}}$ para detectar pontos aberrantes.

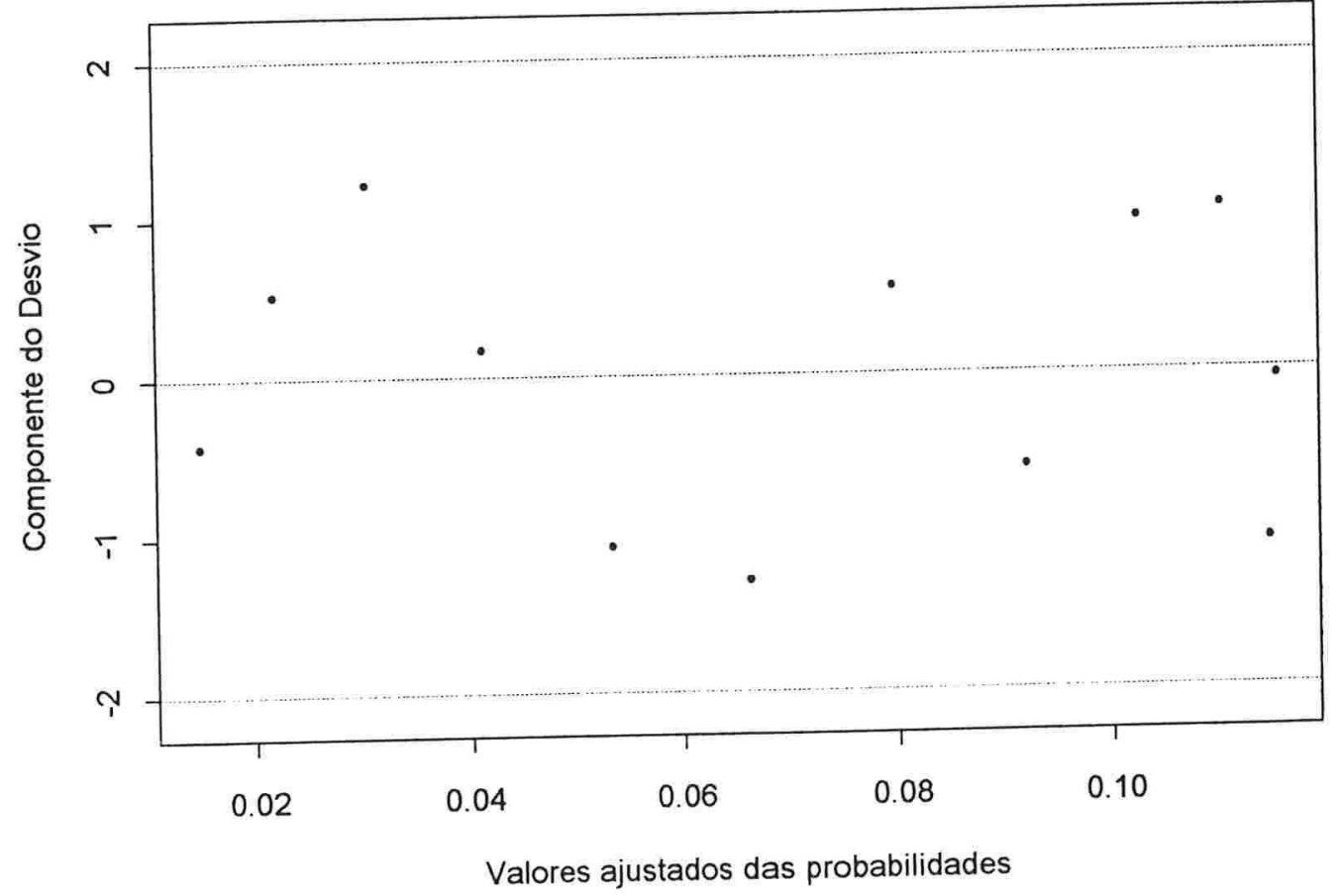


Gráfico 3.1.5: Gráfico do $L D_{i}$ para detectar pontos influentes.

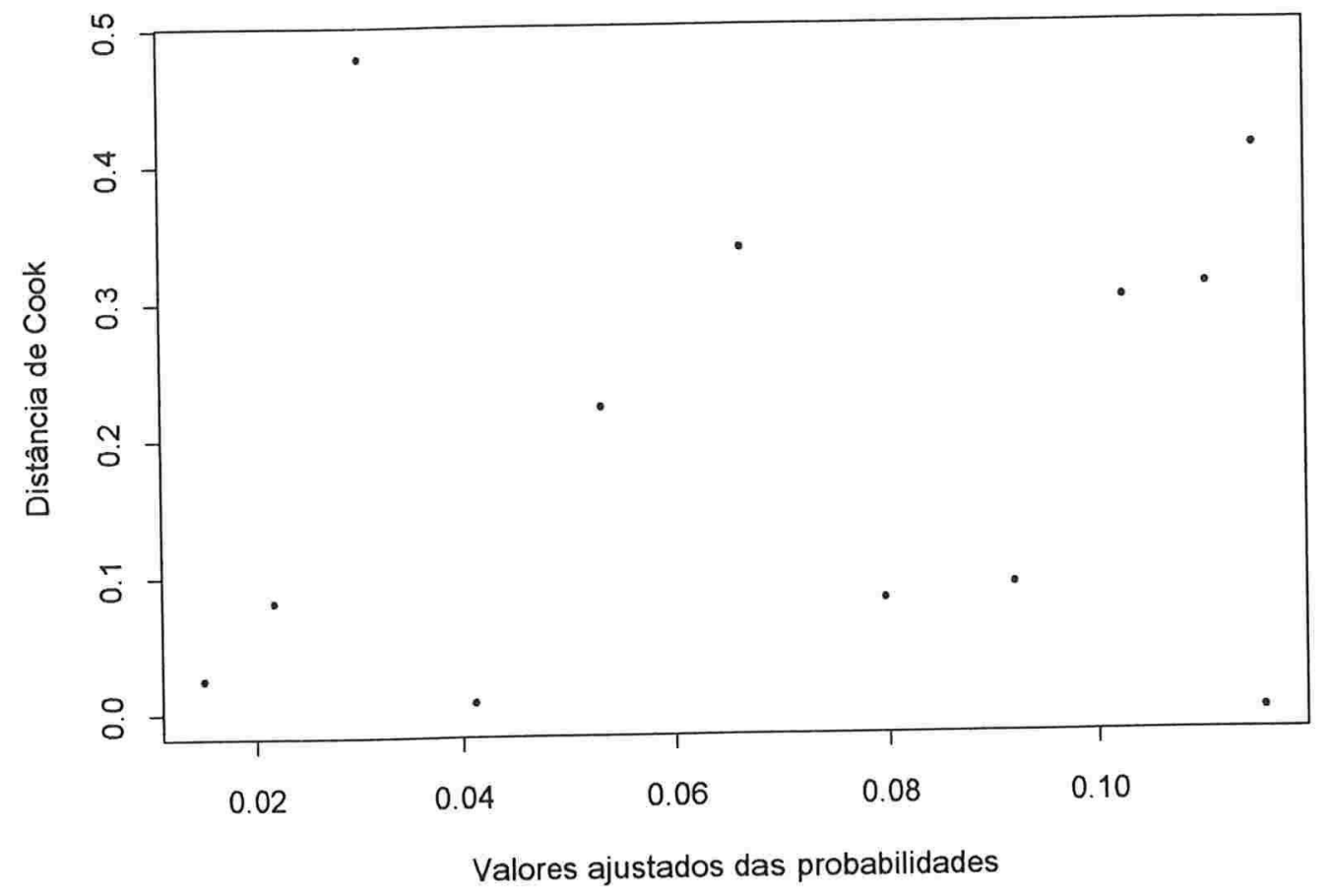

Gráfico 3.1.6: Resíduos padronizados e envelopes.

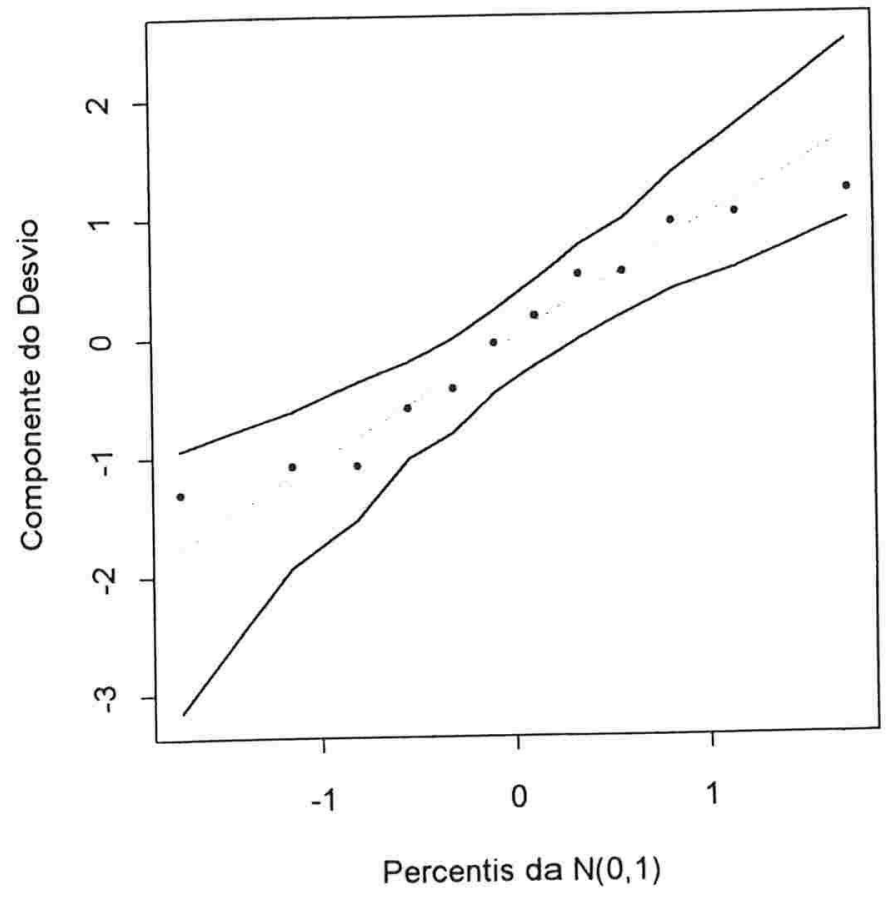




\subsection{Estudo seriado com ratos}

Esse exemplo provém de um estudo com objetivo de avaliar a malignidade de um tipo de tumor de boca humana. Para tanto, um total de 204 ratos tiveram o tumor inoculado e para cada um, observou-se se ocorreu ou não o desenvolvimento da massa tumoral e o tempo de observação em dias até a caquexia, caracterizado pela magreza excessiva que os levavam à morte. Esses dados, analisados pelo Centro de Estatística Aplicada da USP (Paula, Barbosa e Ferreira, 1989), estão apresentados no Apêndice A, no formato em que foram usados neste trabalho. O objetivo aqui será analisar o risco de morte ao longo do tempo comparando os ratos que desenvolveram massa tumoral com os que não desenvolveram. Através dos Gráficos 3.2.1 e 3.2.2 nota-se que o risco observado de morte aumenta até o centésimo dia para os dois grupos.

Novamente procurou-se ajustar o modelo linear, e comparar com alguns modelos com mais parâmetros, como o quadrático, cúbico e cúbico-linear. O ponto de inflexão da equação referente ao modelo cúbico-linear foi determinado como indicado no Capítulo 2. Ajustou-se modelos com pontos de inflexão de 10 a 80, de 10 em 10, e observou-se a soma dos desvios para os dois grupos, como pode ser visto na Tabela 3.2.1. Assim, o modelo cúbico-linear que muda a inclinação no ponto 30 dias foi escolhido por apresentar menor soma dos desvios.

Tanto o modelo cúbico-linear mais adequado quanto os modelos quadrático e cúbico foram comparados com o linear através da estatística da razão de verossimilhança. Como já foi dito no capítulo anterior, é interessante escolher o mesmo modelo para os dois grupos de tal forma que eles possam ser comparados, mesmo que empiricamente. A Tabela 3.2.2a contém as estatísticas do desvio para cada modelo e as estatísticas que comparam os modelos com mais parâmetros com o linear. Da mesma forma, a Tabela 3.2.2b contém as estatísticas de Hosmer e Lemeshow que foram calculadas considerando-se 9 subgrupos tanto para o grupo sem massa quanto para o grupo com massa segundo descrito na Seção 2.2. 
Tabela 3.2.1: Ajustes dos modelos logísticos cúbico-lineares para vários pontos de inflexão nos dois grupos.

\begin{tabular}{|c|c|c|c|c|c|c|c|}
\hline \multirow[b]{3}{*}{$\begin{array}{l}\text { Ponto de } \\
\text { inflexão }\end{array}$} & \multicolumn{6}{|c|}{ Grupo } & \multirow[b]{3}{*}{$\begin{array}{c}\text { Soma dos } \\
\text { desvios }\end{array}$} \\
\hline & \multicolumn{3}{|c|}{ Sem massa tumoral } & \multicolumn{3}{|c|}{ Com massa tumoral } & \\
\hline & Desvio & $\mathrm{gl}$ & $\begin{array}{c}\text { Nivel } \\
\text { descritivo }\end{array}$ & Desvio & $\mathrm{gl}$ & $\begin{array}{c}\text { Nivel } \\
\text { descritivo }\end{array}$ & \\
\hline 80 & 33,406 & 24 & 0,096 & 25,830 & 23 & 0,309 & 59,236 \\
\hline 70 & 32,531 & 24 & 0,114 & 25,983 & 23 & 0,302 & 58,514 \\
\hline 60 & 31,739 & 24 & 0,134 & 26,611 & 23 & 0,273 & 58,350 \\
\hline 50 & 31,588 & 24 & 0,138 & 26,809 & 23 & 0,264 & 58,397 \\
\hline 40 & 30,648 & 24 & 0,164 & 26,354 & 23 & 0,284 & 57,002 \\
\hline 30 & 30,193 & 24 & 0,178 & 26,032 & 23 & 0,299 & 56,225 \\
\hline 20 & 29,848 & 24 & 0,190 & 27,677 & 23 & 0,228 & 57,525 \\
\hline 10 & 35,817 & 24 & 0,057 & 29,785 & 23 & 0,156 & 65,602 \\
\hline
\end{tabular}

Tabela 3.2.2a: Ajustes dos modelos logísticos para os dois grupos, com massa tumoral e sem massa tumoral, segundo a função desvio.

\begin{tabular}{|c|c|c|c|c|c|c|c|}
\hline Grupo & Modelo & g.l. & Desvio & $\begin{array}{c}\text { Nivel } \\
\text { descritivo }\end{array}$ & $\begin{array}{c}\text { Diferença } \\
\text { dos } \\
\text { desvios }\end{array}$ & $\begin{array}{c}\text { g.l. } \\
\text { (diferença) }\end{array}$ & $\begin{array}{c}\text { Nível } \\
\text { descritivo } \\
\text { (diferença) }\end{array}$ \\
\hline \multirow{4}{*}{ Sem massa } & linear & 26 & 39,356 & 0,045 & & & \\
\hline & quadrático & 25 & 33,267 & 0,125 & 6,089 & 1 & 0,014 \\
\hline & cúbico & 24 & 33,267 & 0,099 & 6,089 & 2 & 0,048 \\
\hline & cúbico-linear & 24 & 30,193 & 0,178 & 9,163 & 2 & 0,010 \\
\hline \multirow{4}{*}{ Com massa } & linear & 25 & 30,947 & 0,191 & & & \\
\hline & quadrático & 24 & 25,586 & 0,374 & 5,361 & 1 & 0,021 \\
\hline & cúbico & 23 & 25,579 & 0,321 & 5,368 & 2 & 0,068 \\
\hline & cúbico-linear & 23 & 26,032 & 0,299 & 4,915 & 2 & 0,086 \\
\hline
\end{tabular}


Tabela 3.2.2b: Ajustes dos modelos logísticos para os dois grupos, com massa tumoral e sem massa tumoral, segundo a estatística de Hosmer e Lemeshow.

\begin{tabular}{|c|c|c|c|c|}
\hline Grupo & Modelo & g.l. & $\begin{array}{c}\text { Estatistica de Hosmer e } \\
\text { Lemeshow }\end{array}$ & $\begin{array}{c}\text { Nivel } \\
\text { descritivo }\end{array}$ \\
\hline \multirow{4}{*}{ Sem massa } & linear & 7 & 12,044 & 0,099 \\
\hline & quadrático & 7 & 8,574 & 0,285 \\
\hline & cúbico & 7 & 8,597 & 0,283 \\
\hline & cúbico-linear & 7 & 9,279 & 0,233 \\
\hline \multirow{4}{*}{ Com massa } & linear & 7 & 6,361 & 0,498 \\
\hline & quadrático & 7 & 7,952 & 0,337 \\
\hline & cúbico & 7 & 3,424 & 0,843 \\
\hline & cúbico-linear & 7 & 4,693 & 0,697 \\
\hline
\end{tabular}

Tanto para o grupo sem massa tumoral quanto para o grupo com massa tumoral, os modelos com mais parâmetros parecem ser mais adequados do que o linear, de acordo com as estatísticas da razão de verossimilhança apresentadas na Tabela 3.2.2a. Quando olhados conjuntamente, não parece haver um modelo que seja o melhor nos dois grupos e portanto optou-se por adotar o modelo quadrático que é o menos parametrizado entre os que apresentaram bom ajuste

As Tabelas 3.2 .3 e 3.2 .4 contém as estimativas dos parâmetros do modelo ajustado para cada grupo.

Tabela 3.2.3: Estimativas dos parâmetros do modelo quadrático para o grupo de ratos que não desenvolveu massa tumoral.

\begin{tabular}{lccc}
\hline \multicolumn{1}{c}{ Coeficientes } & Estimativa & Desvio padrão & $\begin{array}{c}\text { Valor da } \\
\text { estatística } Z\end{array}$ \\
\hline intercepto & $-6,4808$ & 0,9314 & $-6,9576$ \\
coeficiente linear & 0,0908 & 0,0315 & 2,8845 \\
coeficiente quadrático & $-0,0005$ & 0,0002 & $-2,1044$ \\
\hline
\end{tabular}


Tabela 3.2.4: Estimativas dos parâmetros do modelo quadrático para o grupo de ratos que desenvolveu massa tumoral.

\begin{tabular}{lccc}
\hline \multicolumn{1}{c}{ Coeficientes } & Estimativa & Desvio padrão & $\begin{array}{c}\text { Valor da } \\
\text { estatística } Z\end{array}$ \\
\hline intercepto & $-8,8004$ & 1,5515 & $-5,6719$ \\
coeficiente linear & 0,1334 & 0,0464 & 2,8748 \\
coeficiente quadrático & $-0,0006$ & 0,0003 & $-1,9706$ \\
\hline
\end{tabular}

As Tabelas 3.2 .5 e 3.2 .6 contém as estimativas da taxa de risco e da função de sobrevivência e seus respectivos desvios-padrão aproximados para alguns dos intervalos segundo o modelo quadrático para os dois grupos estudados.

Tabela 3.2.5: Estimativas para taxa de risco e função de sobrevivência e seus respectivos desvios-padrão aproximados para alguns dos intervalos no modelo quadrático referente ao grupo sem massa.

\begin{tabular}{ccccc}
\hline Dias & $\begin{array}{c}\text { Taxa de } \\
\text { risco }\end{array}$ & $\begin{array}{c}\text { Desvio } \\
\text { padrão }\end{array}$ & $\begin{array}{c}\text { Função de } \\
\text { sobrevivência }\end{array}$ & $\begin{array}{c}\text { Desvio } \\
\text { padrão }\end{array}$ \\
\hline 5 & 0,0019 & 0,0016 & 0,9981 & 0,0016 \\
10 & 0,0029 & 0,0021 & 0,9951 & 0,0038 \\
15 & 0,0043 & 0,0026 & 0,9908 & 0,0064 \\
20 & 0,0063 & 0,0032 & 0,9845 & 0,0095 \\
25 & 0,0090 & 0,0037 & 0,9756 & 0,0130 \\
30 & 0,0124 & 0,0043 & 0,9636 & 0,0168 \\
35 & 0,0165 & 0,0050 & 0,9475 & 0,0209 \\
40 & 0,0216 & 0,0059 & 0,9271 & 0,0251 \\
45 & 0,0275 & 0,0070 & 0,9016 & 0,0295 \\
50 & 0,0339 & 0,0085 & 0,8709 & 0,0343 \\
70 & 0,0609 & 0,0156 & 0,7059 & 0,0572 \\
90 & 0,0731 & 0,0219 & 0,5270 & 0,0756 \\
110 & 0,0592 & 0,0331 & 0,4005 & 0,0899 \\
130 & 0,0320 & 0,0347 & 0,3368 & 0,1112 \\
\hline
\end{tabular}


Tabela 3.2.6: Estimativas para taxa de risco e função de sobrevivência e seus respectivos desvios-padrão aproximados para alguns dos intervalos no modelo quadrático referente ao grupo com massa.

\begin{tabular}{cccccc}
\hline Dias & $\begin{array}{c}\text { Taxa de } \\
\text { risco }\end{array}$ & $\begin{array}{c}\text { Desvio } \\
\text { padrão }\end{array}$ & & $\begin{array}{c}\text { Função de } \\
\text { sobrevivência }\end{array}$ & $\begin{array}{c}\text { Desvio } \\
\text { padrão }\end{array}$ \\
\hline 5 & 0,0002 & 0,0003 & & 0,9997 & 0,0003 \\
10 & 0,0004 & 0,0005 & & 0,9994 & 0,0008 \\
15 & 0,0007 & 0,0008 & & 0,9986 & 0,0015 \\
20 & 0,0013 & 0,0011 & & 0,9974 & 0,0026 \\
25 & 0,0022 & 0,0016 & & 0,9952 & 0,0042 \\
30 & 0,0036 & 0,0021 & & 0,9916 & 0,0063 \\
35 & 0,0057 & 0,0028 & & 0,9859 & 0,0089 \\
40 & 0,0088 & 0,0035 & & 0,9772 & 0,0121 \\
45 & 0,0132 & 0,0044 & & 0,9643 & 0,0159 \\
50 & 0,0191 & 0,0056 & & 0,9459 & 0,0202 \\
70 & 0,0586 & 0,0157 & & 0,7967 & 0,0469 \\
90 & 0,1056 & 0,0302 & & 0,5482 & 0,0805 \\
110 & 0,1172 & 0,0614 & & 0,3335 & 0,1032 \\
130 & 0,0932 & 0,0865 & & 0,2212 & 0,1342 \\
\hline
\end{tabular}

Analisando as tabelas acima e os Gráficos 3.2 .3 e 3.2.4, que contém as curvas ajustadas da taxa de risco e da função de sobrevivência de cada grupo, nota-se que o risco de morte no grupo de ratos sem massa tumoral parece ser maior no início do seguimento. Esse resultado parece em princípio contraditório, mas pode ser justificado biológicamente, pois os ratos que não desenvolveram massa tumoral podem ter sofrido algum tipo de esgotamento devido a sua forte resistência, o que levou boa parte deles a morte antes dos que desenvolveram massa tumoral. Aqueles que sobreviveram ao início do estudo tornaram-se mais fortes, o que explica o fato de que, após aproximadamente 70 , dias o risco de morte ser maior entre os ratos com massa tumoral.

Como consequência do comportamento da taxa de risco, a sobrevida no grupo com massa tumoral é maior no início do seguimento perdendo essa vantagem ao longo do tempo. 
Os Gráficos 3.2.5 e 3.2.6 mostram as curvas referentes às taxas de risco e as bandas com $95 \%$ de confiança em cada grupo. Analogamente, os Gráficos 3.2 .7 e 3.2 .8 mostram as curvas de sobrevida e as bandas com $95 \%$ de confiança também para cada grupo.

Com intuito de estabelecer uma relação quantitativa entre as chances de morte no dois grupos, considerou-se, assim como no Capítulo 2, uma medida equivalente à razão das chances. Constatou-se que na ocasião do quinto dia, a chance de morte no grupo sem massa tumoral é 8,24 vezes maior do que no grupo com massa tumoral. Essa vantagem do grupo com massa tumoral diminui ao longo do tempo e no final, com 110 dias de seguimento, a chance de morte no grupo com massa tumoral já é 3,18 vezes maior do que no grupo sem massa tumoral.

Para cada um dos modelos ajustados, foi feita uma análise de resíduos. Os Gráficos 3.2 .9 e 3.2 .12 do componente do desvio padronizado contra os valores ajustados da taxa de risco, indicam que, nos dois grupos, a maior parte dos pontos estão dentro dos limites toleráveis. Já o Gráfico 3.2 .10 da distância de Cook pelos valores ajustados das probabilidades para o grupo sem massa tumoral, destaca o ponto referente ao intervalo 10 , que, quando presente nos dados, altera os coeficientes do modelo ajustado em mais de $10 \%$. Da mesma forma, para o grupo com massa tumoral, o Gráfico 3.2.13 destaca o ponto referente ao intervalo 22 , que altera em $53 \%$ a estimativa do coeficiente quadrático. Em ambos os casos ocorreu proporcionalmente mais eventos do que nos intervalos próximos como pode ser visto pelos Gráficos 3.2 .1 e 3.2 .2 da taxa de risco observada. A partir do Gráfico 3.2.11, que contém os resíduos padronizados e envelopes para o grupo sem massa tumoral, nota-se que alguns pontos encontram-se fora das bandas, além de existir indícios de superdispersão, já que os resíduos negativos estão mais próximos da banda inferior e os positivos mais próximos da banda superior. Já para o grupo com massa tumoral, o Gráfico 3.2.14, mostra poucos pontos fora das bandas e, além disso, não parece haver alguma tendência que indique superdispersão. 
Gráfico 3.2.1: Taxas de risco observada para o grupo sem massa.

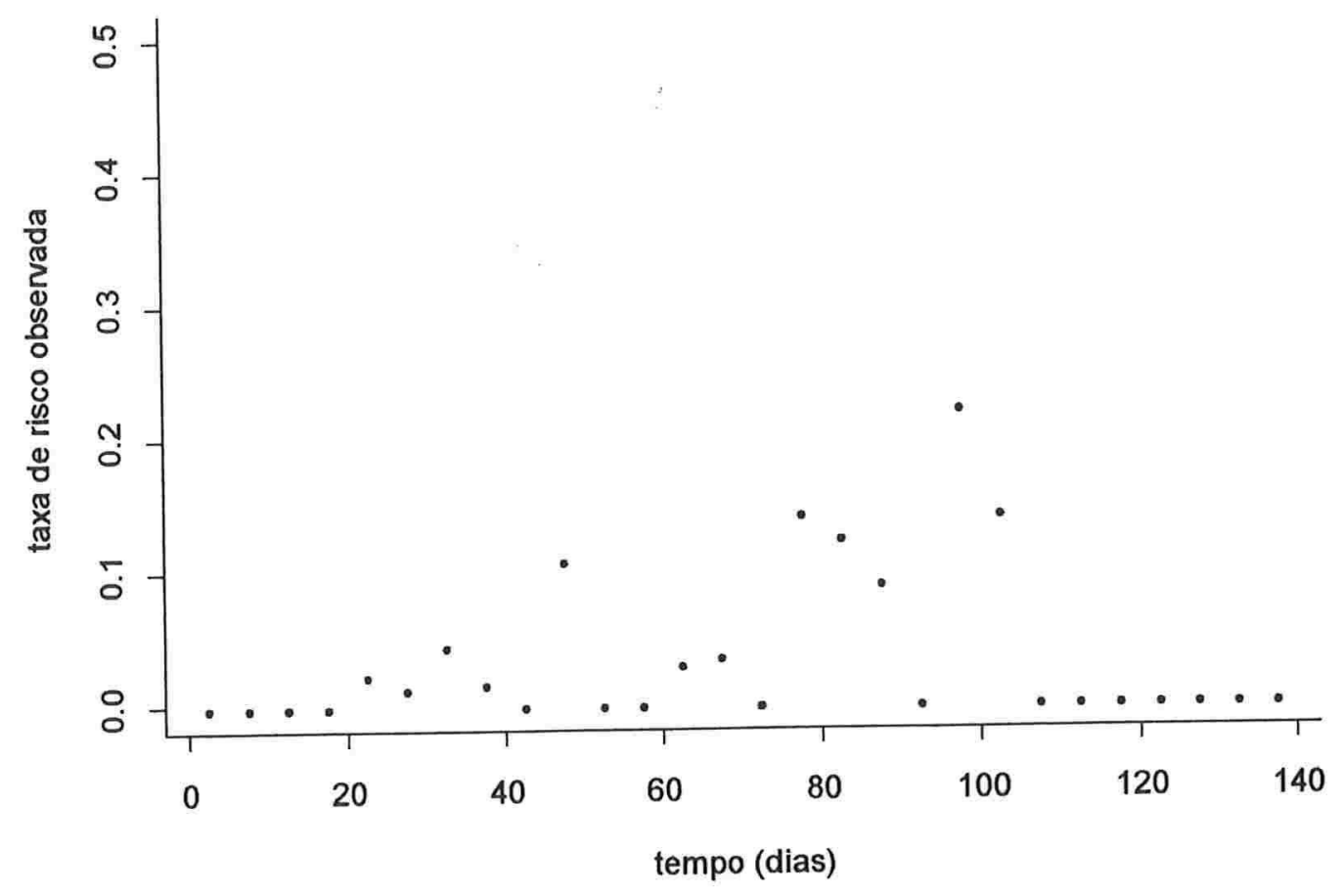

Gráfico 3.2.2: Taxas de risco observada para o grupo com massa.

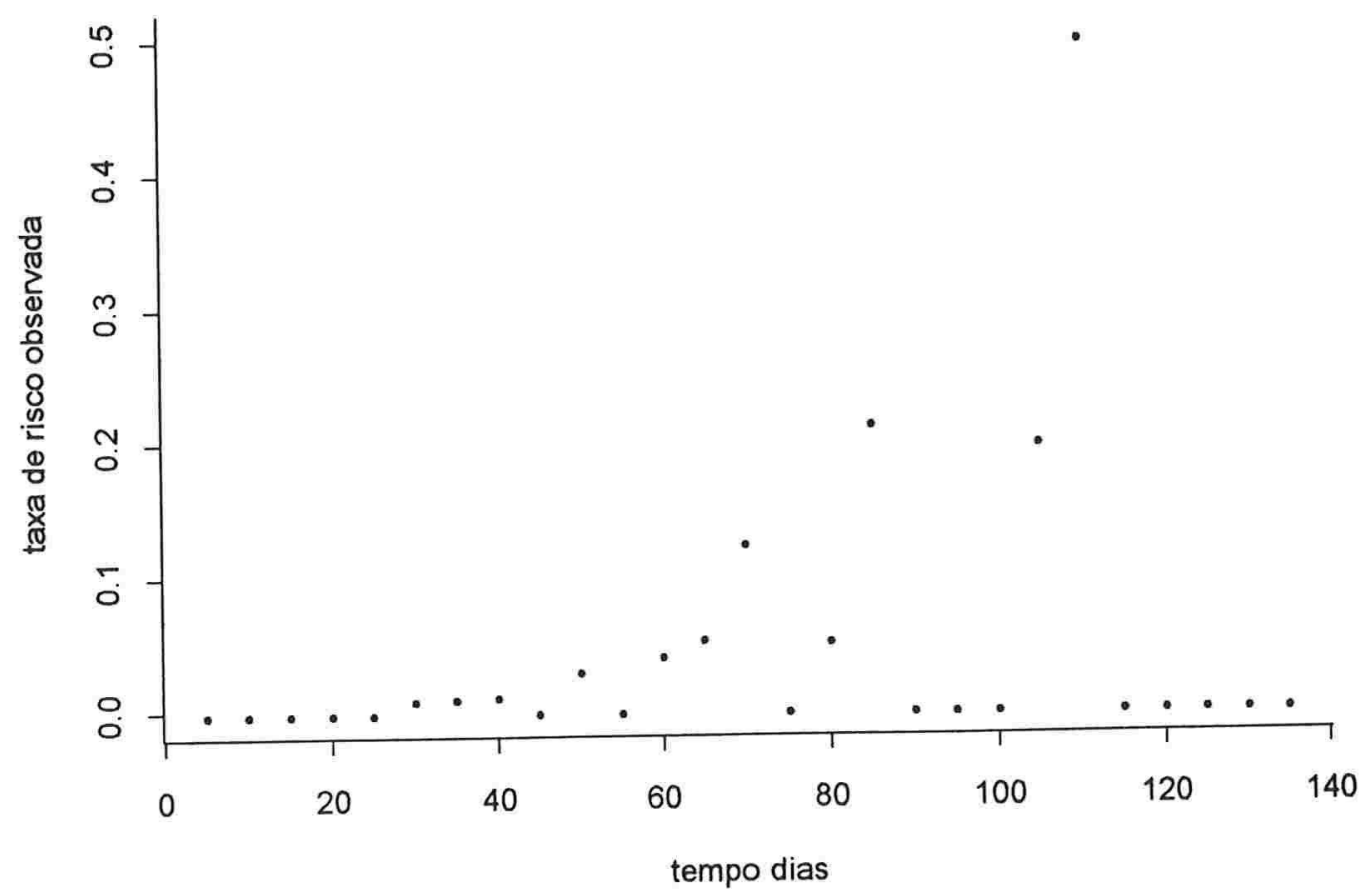


Gráfico 3.2.3: Taxas de risco estimada referente ao modelo quadrático.

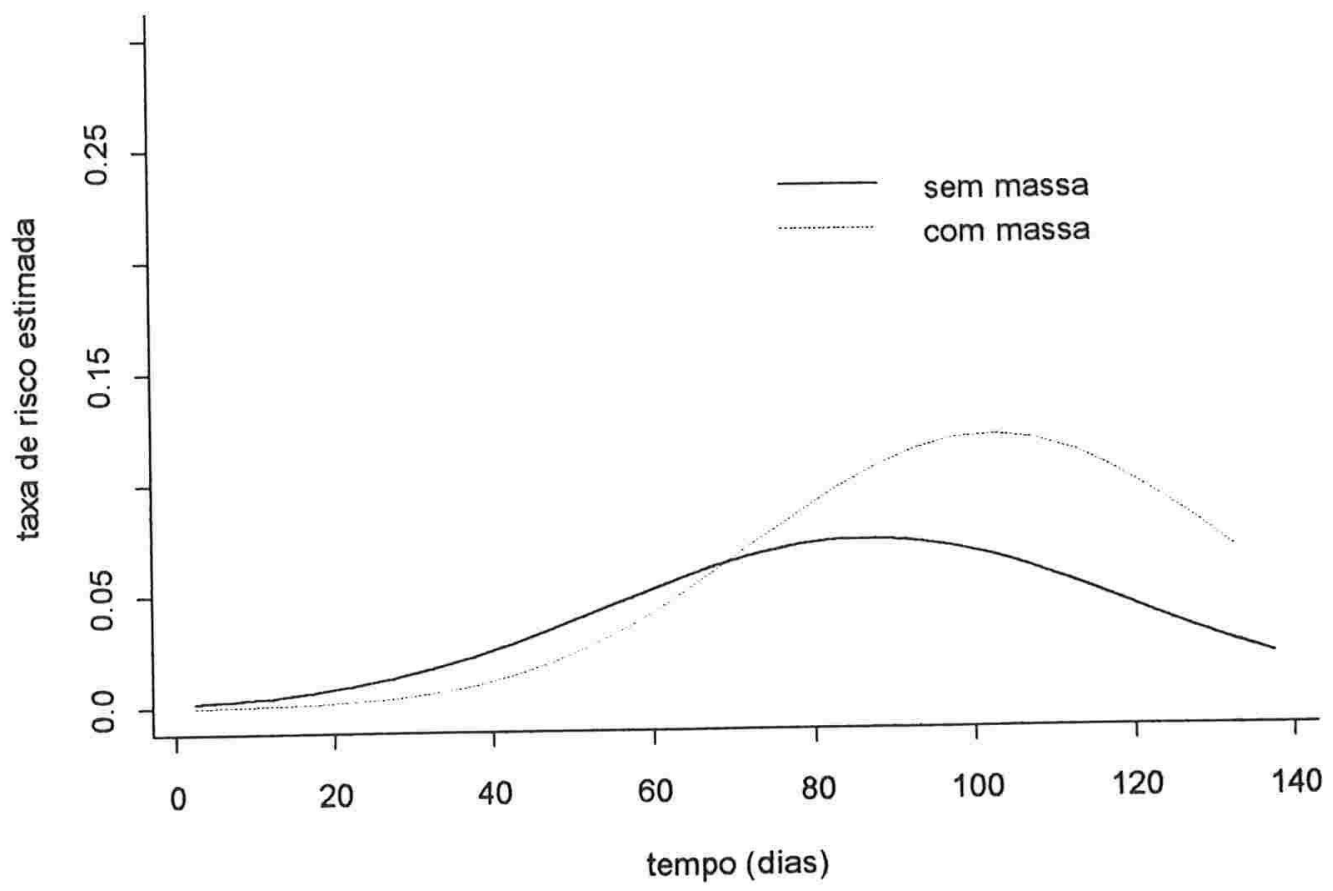

Gráfico 3.2.4: Curvas de sobrevidas estimada referente ao modelo quadrático.

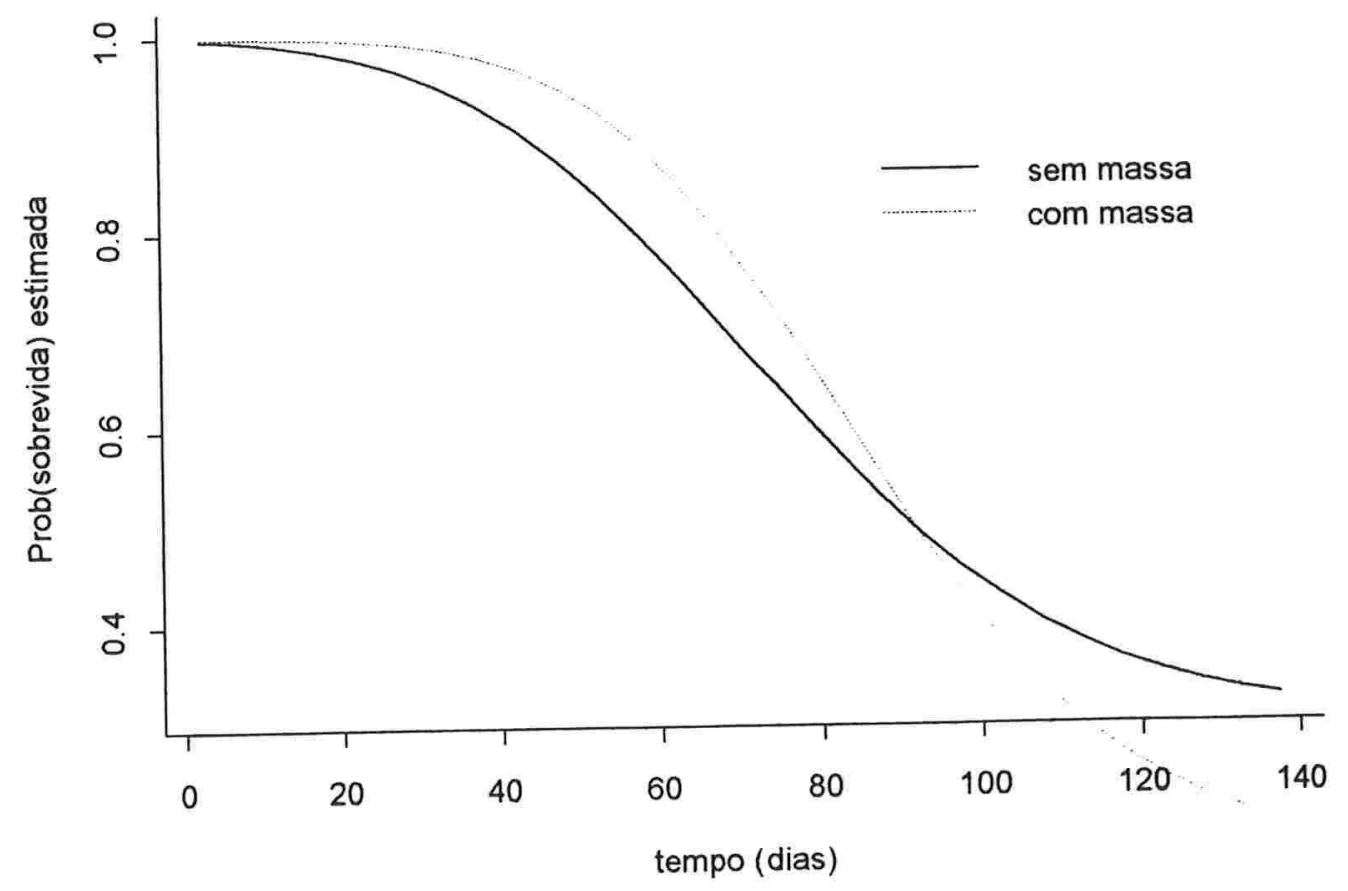


Gráfico 3.2.5: Taxa de risco e bandas de confiança (95\%) estimadas no grupo sem massa tumoral.

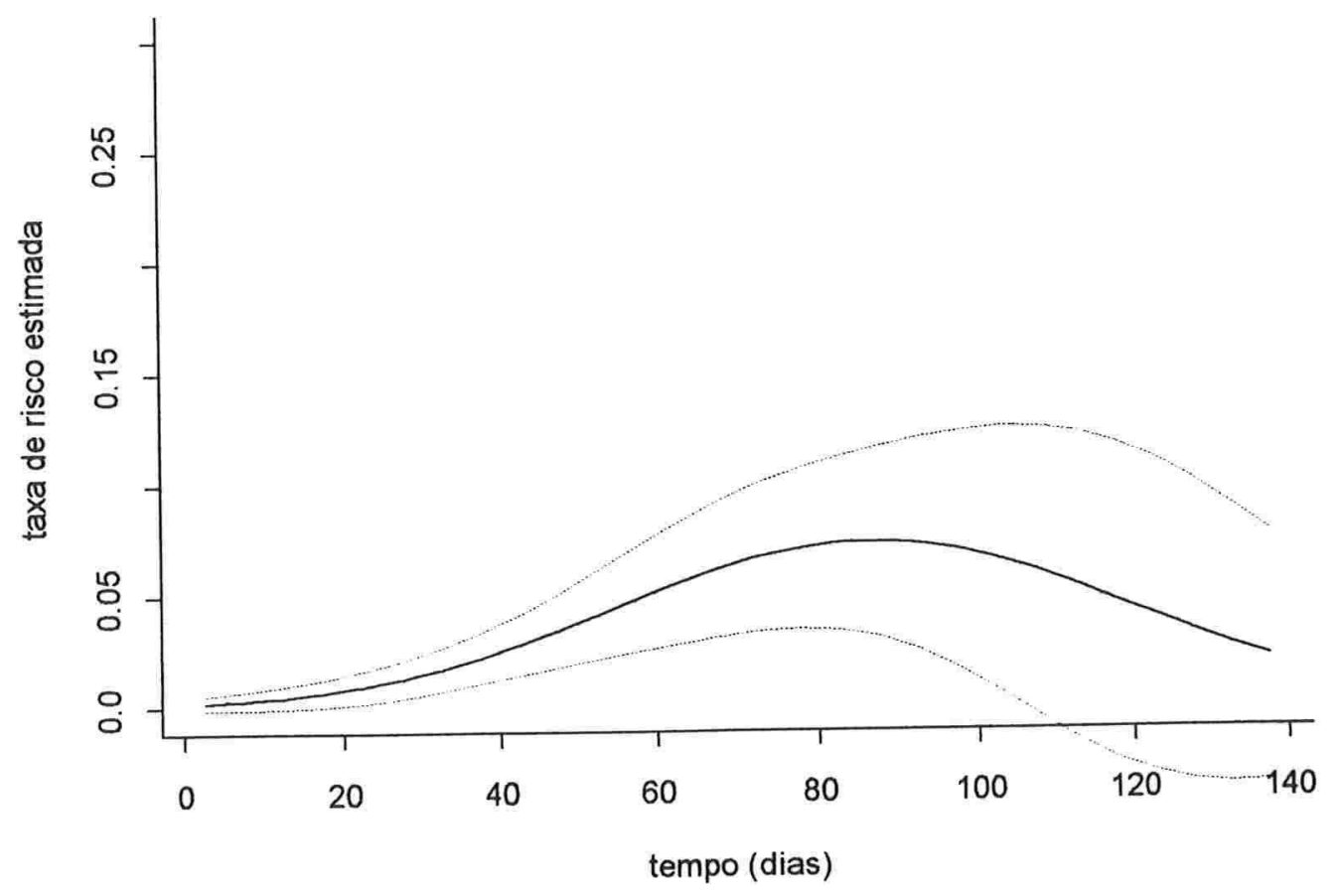

Gráfico 3.2.6: Taxa de risco e bandas de confiança (95\%) estimadas no grupo com massa tumoral.

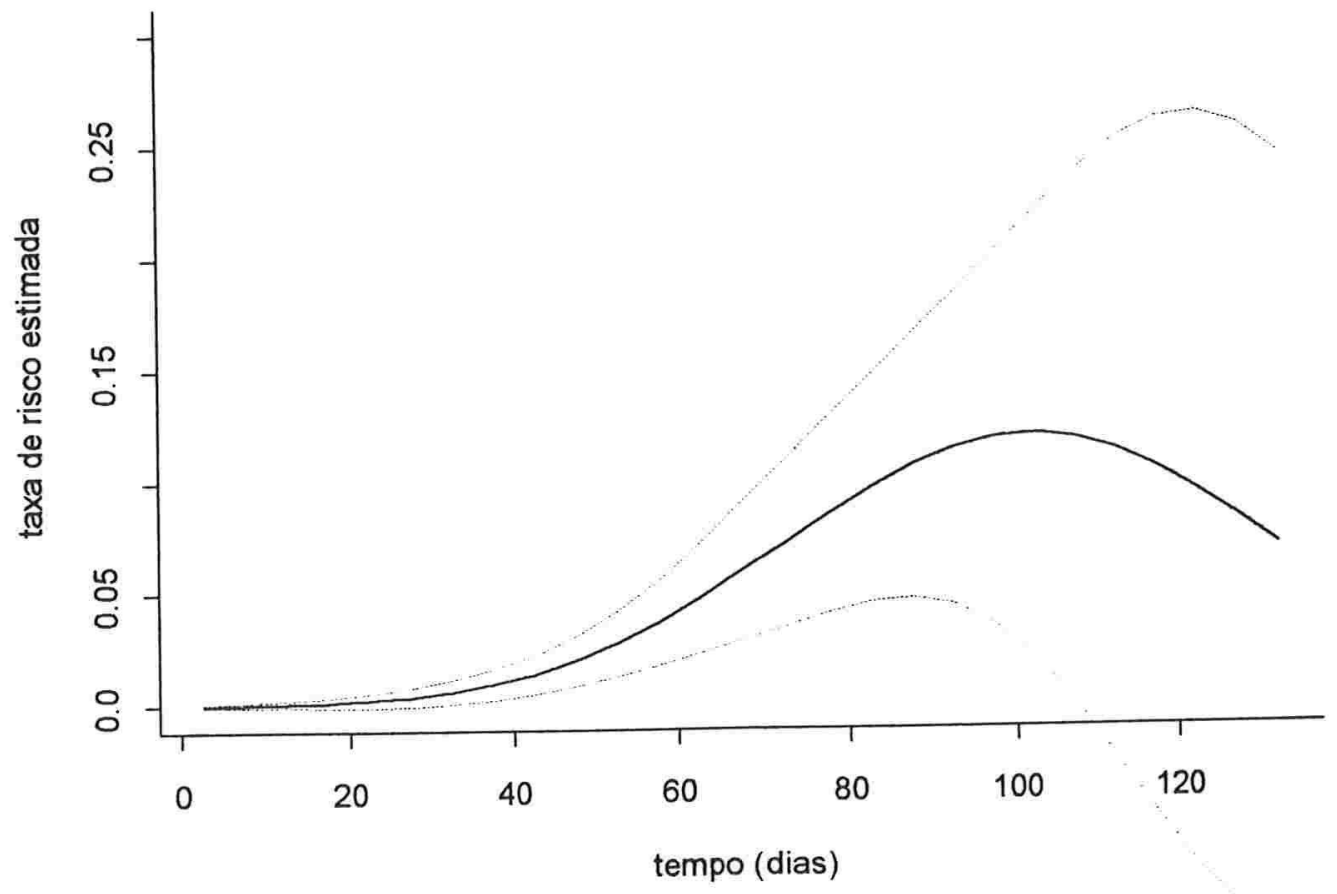


Gráfico 3.2.7: Curva de sobrevida e bandas de confiança (95\%) estimadas no grupo sem massa tumoral.

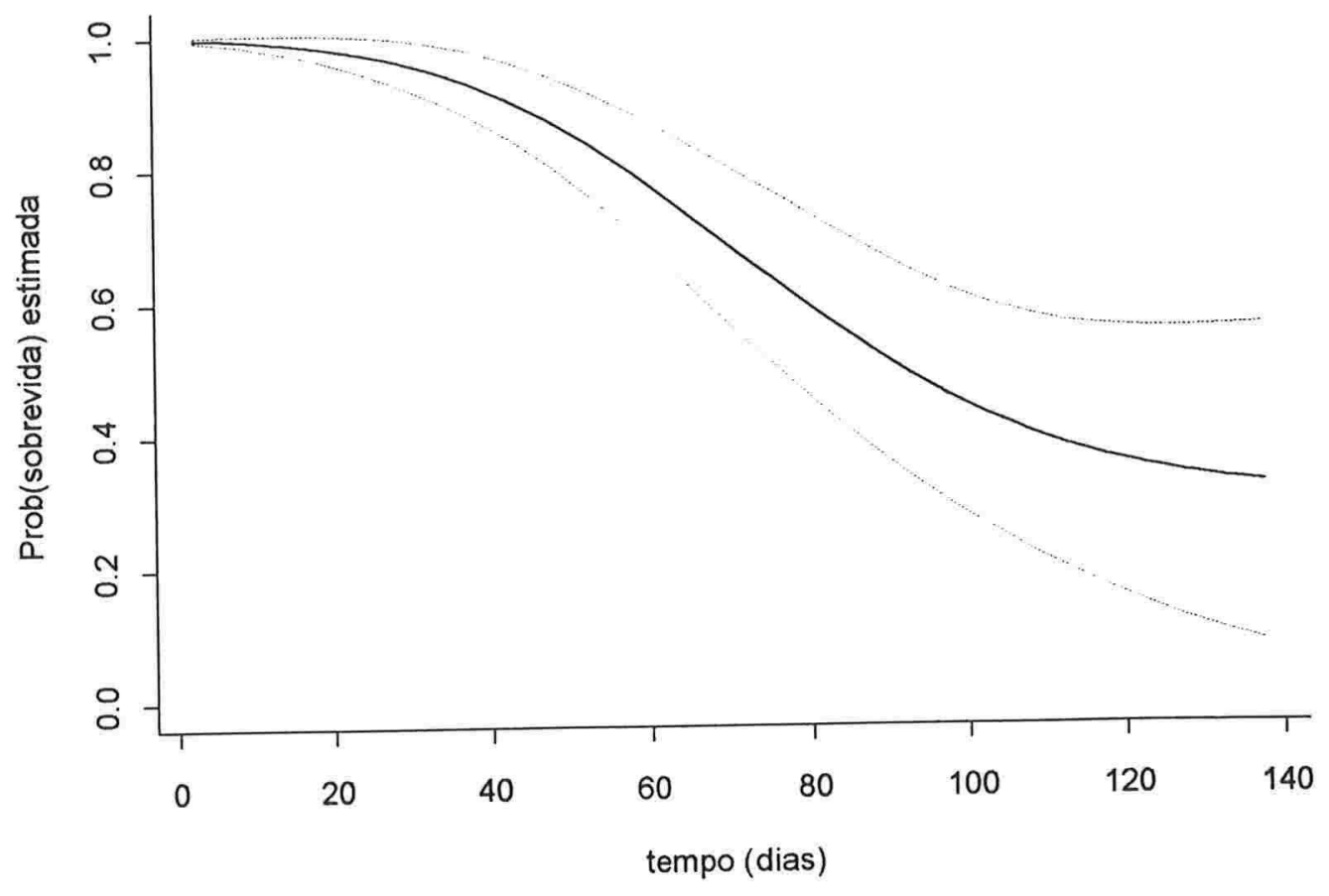

Gráfico 3.2.8: Curva de sobrevida e bandas de confiança (95\%) estimadas no grupo com massa tumoral.

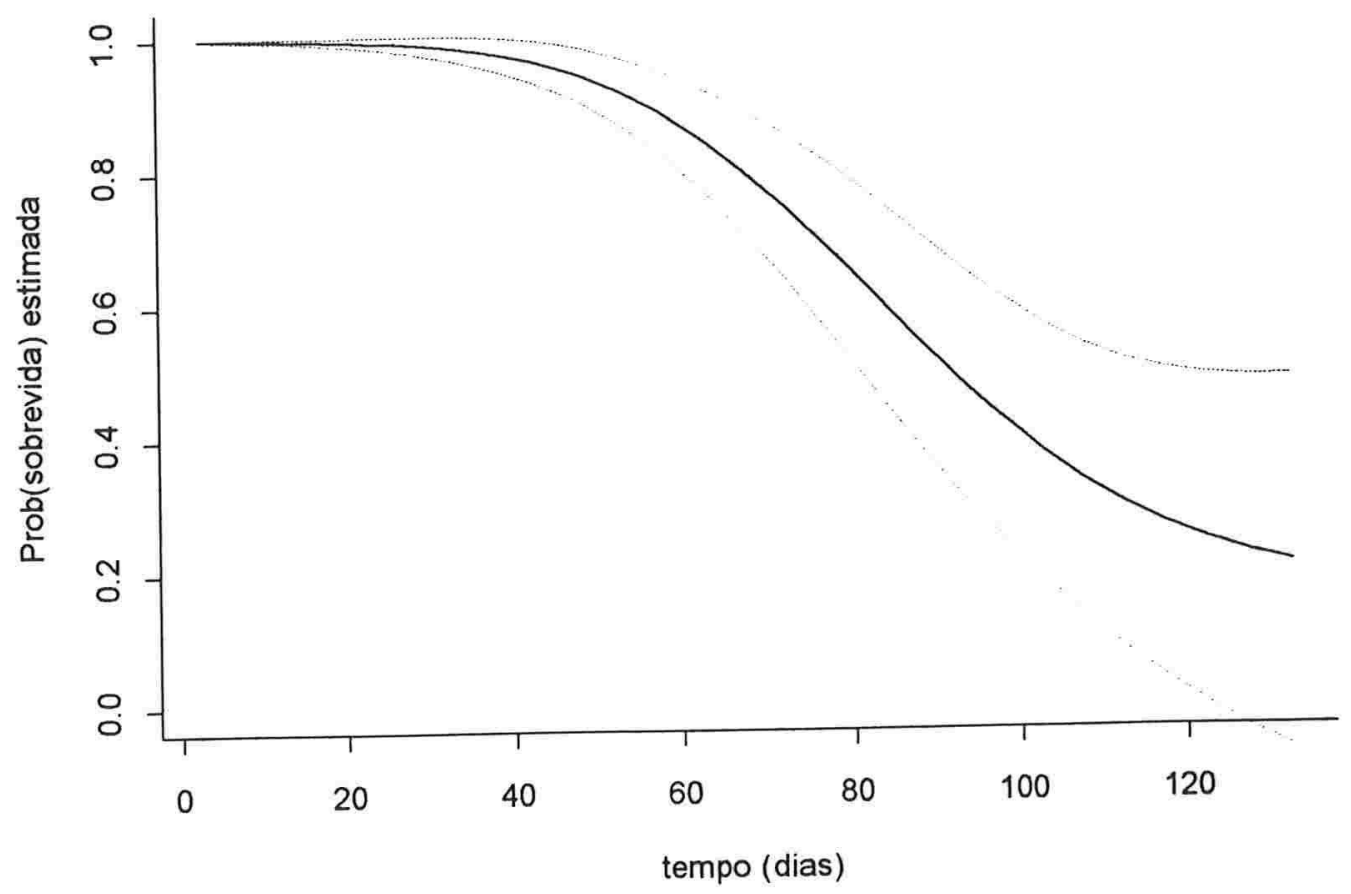


Gráfico 3.2.9: Gráfico do $t_{D_{i}}$ para detectar pontos aberrantes no grupo sem massa tumoral.

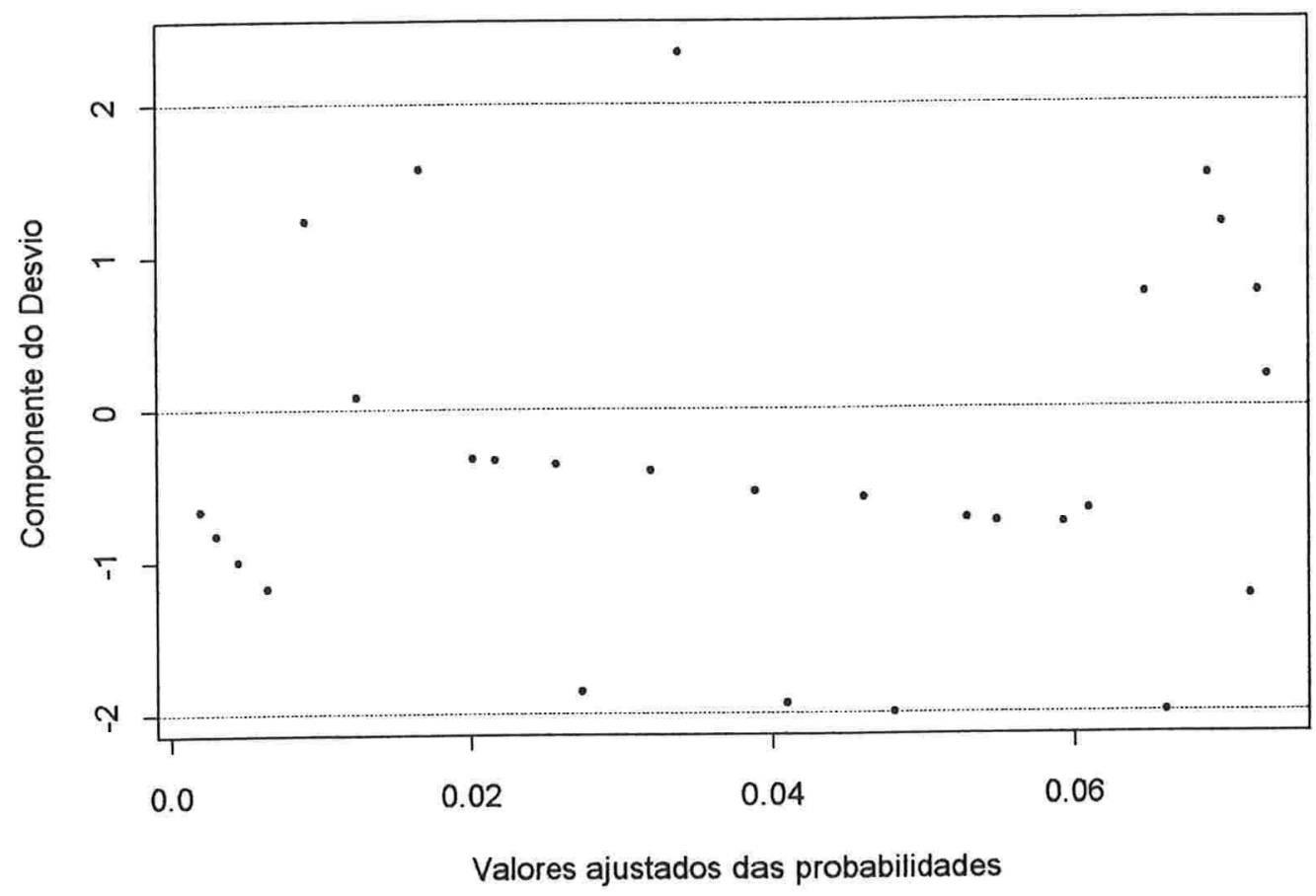

Gráfico 3.2.10: Gráfico do $L D_{i}$ para detectar pontos influentes no grupo sem massa tumoral.

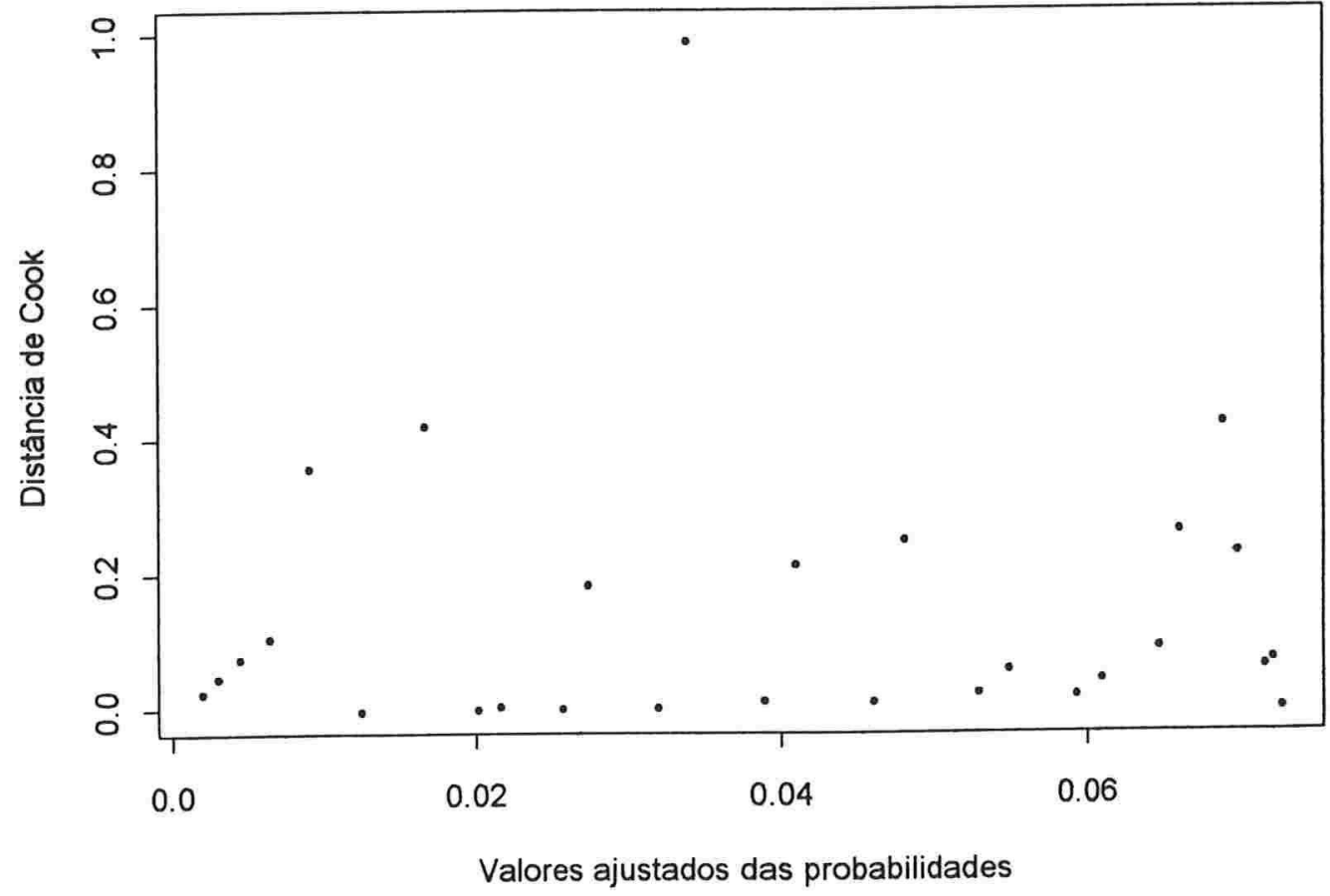


Gráfico 3.2.11: Resíduos padronizados e envelopes para o grupo sem massa tumoral.

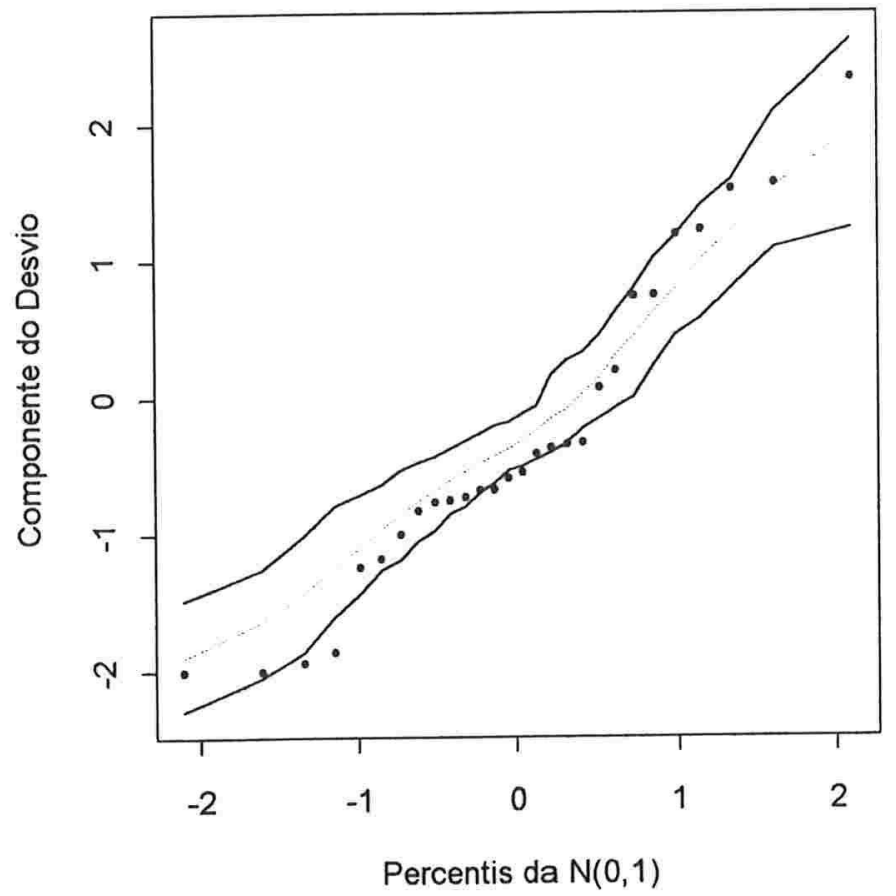

Gráfico 3.2.12: Gráfico do $t_{D_{1}}$ para detectar pontos aberrantes no grupo com massa tumoral.

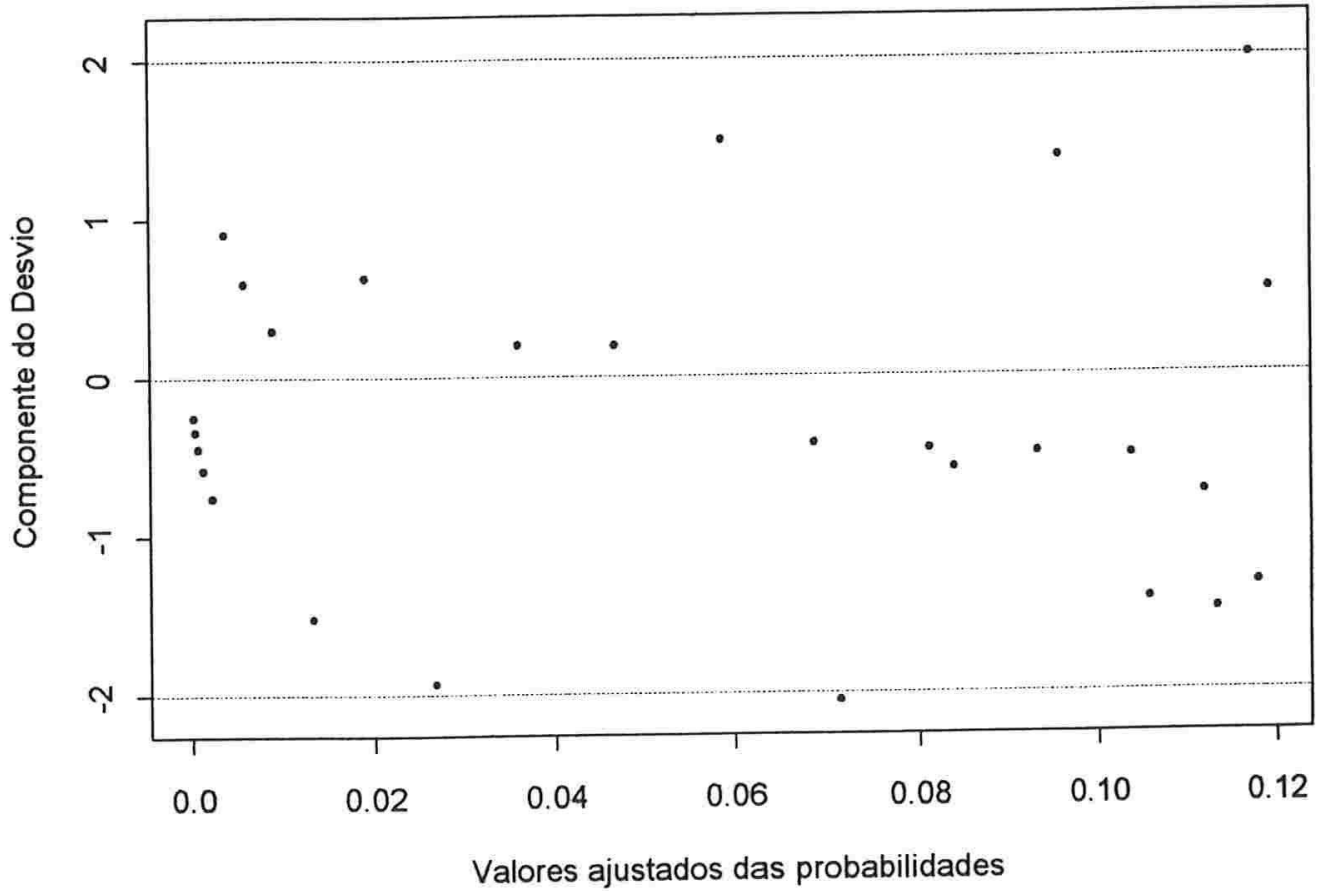


Gráfico 3.2.13: Gráfico do $L D_{i}$ para detectar pontos influentes no grupo com massa tumoral.

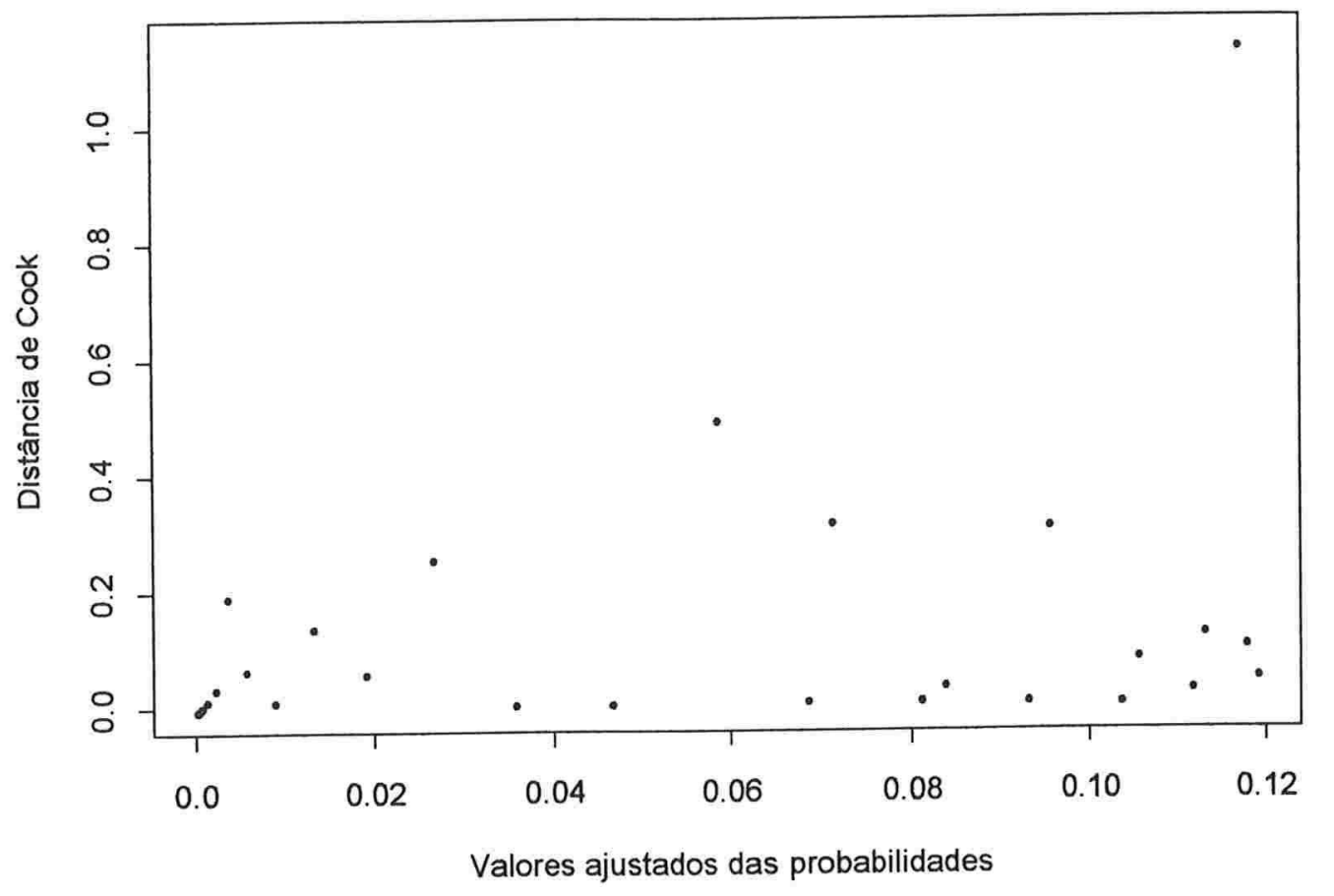

Gráfico 3.2.14: Resíduos padronizados e envelopes no grupo com massa tumoral.

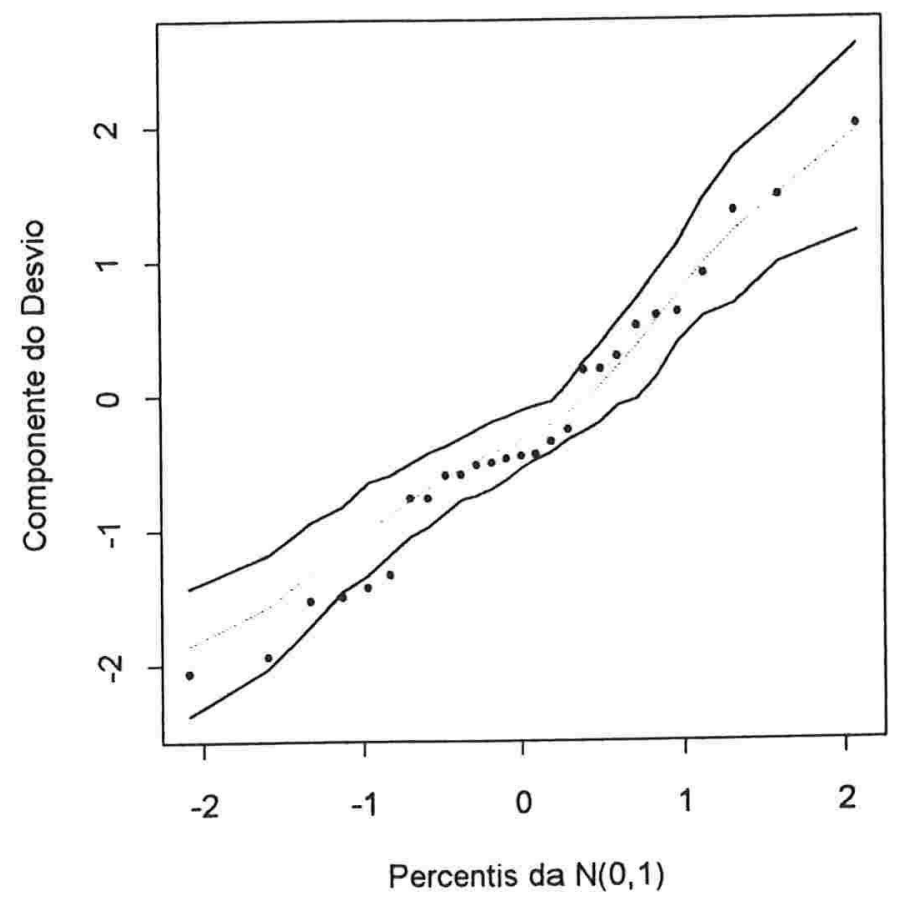


Com o intuito de modelar a superdispersão detectada no grupo sem massa tumoral ajustou-se um modelo de quasi-verossimilhança, e o parâmetro de dispersão foi estimado por 1,200, o que não parece ser alto. As estimativas corrigidas dos parâmetros e dos desviospadrão da taxa de risco e função de sobreviência estão apresentados a seguir nas tabelas 3.2.7 e 3.2.8.

Tabela 3.2.7: Estimativas dos parâmetros do modelo de quasiverossimilhança quadrático para o grupo de ratos que não desenvolveu massa tumoral.

\begin{tabular}{lccc}
\hline \multicolumn{1}{c}{ Coeficientes } & Estimativa & Desvio padrão & $\begin{array}{c}\text { Valor da } \\
\text { estatística } Z\end{array}$ \\
\hline intercepto & $-6,4808$ & 1,0207 & $-6,3491$ \\
coeficiente linear & 0,0908 & 0,0345 & 2,6323 \\
coeficiente quadrático & $-0,0005$ & 0,0003 & $-1,9204$ \\
\hline
\end{tabular}

Tabela 3.2.8: Estimativas para taxa de risco e função de sobrevivência e seus respectivos desvios-padrão aproximados para alguns dos intervalos no modelo de quasi-verossimilhança quadrático referente ao grupo sem massa.

\begin{tabular}{ccccc}
\hline Dias & $\begin{array}{c}\text { Taxa de } \\
\text { risco }\end{array}$ & $\begin{array}{c}\text { Desvio } \\
\text { padrão }\end{array}$ & $\begin{array}{c}\text { Função de } \\
\text { sobrevivência }\end{array}$ & $\begin{array}{c}\text { Desvio } \\
\text { padrão }\end{array}$ \\
\hline 5 & 0,0019 & 0,0018 & 0,9981 & 0,0018 \\
10 & 0,0029 & 0,0023 & 0,9951 & 0,0041 \\
15 & 0,0043 & 0,0029 & 0,9908 & 0,0070 \\
20 & 0,0063 & 0,0035 & 0,9845 & 0,0104 \\
25 & 0,0090 & 0,0041 & 0,9756 & 0,0142 \\
30 & 0,0124 & 0,0048 & 0,9636 & 0,0184 \\
35 & 0,0165 & 0,0055 & 0,9475 & 0,0229 \\
40 & 0,0216 & 0,0064 & 0,9271 & 0,0275 \\
45 & 0,0275 & 0,0077 & 0,9016 & 0,0323 \\
50 & 0,0339 & 0,0093 & 0,8709 & 0,0375 \\
70 & 0,0609 & 0,0171 & 0,7059 & 0,0626 \\
90 & 0,0731 & 0,0239 & 0,5270 & 0,0828 \\
110 & 0,0592 & 0,0363 & 0,4005 & 0,0984 \\
130 & 0,0320 & 0,0380 & 0,3368 & 0,1218 \\
\hline
\end{tabular}




\subsection{Estudo sobre a sobrevivência a câncer no ovário após a segunda laparotomia.}

Um estudo de seguimento foi feito com 114 mulheres com câncer no ovário onde observou-se o tempo de sobrevivência após a segunda laparotomia (Lima, 1996). Um dos interesses do estudo era investigar a eficácia de um certo tratamento, ou seja, verificar se a aplicação desse tratamento prolonga a sobrevida dos pacientes. Para tanto, as pacientes foram divididas em dois grupos: tratadas e não tratadas.

Através dos Gráficos 3.3.1 e 3.3.2, da taxa de risco observada ao longo do tempo para cada grupo, nota-se que o risco de morte é maior nos primeiros anos, sendo que para o grupo não tratado esse risco parece ser ainda maior.

Seguindo os passos da análise, procurou-se inicialmente ajustar o modelo linear por ser o mais simples, e comparar com alguns modelos com mais parâmetros, como o quadrático e cúbico. Foi verificado a qualidade de ajuste dos modelos propostos através da estatística do desvio, na Tabela 3.3.1a, e da estatística de Hosmer e Lemeshow na Tabela 3.3.1b.

Tabela 3.3.1a: Ajustes dos modelos logísticos para os dois grupos, tratado e não tratado segundo a função desvio.

\begin{tabular}{|c|c|c|c|c|c|c|c|}
\hline Grupo & Modelo & g.1. & Desvio & $\begin{array}{c}\text { Nível } \\
\text { descritivo }\end{array}$ & $\begin{array}{c}\text { Diferença } \\
\text { dos } \\
\text { desvios }\end{array}$ & $\begin{array}{c}\text { g.l. } \\
\text { (diferença) }\end{array}$ & $\begin{array}{c}\text { Nivel } \\
\text { descritivo } \\
\text { (diferença) }\end{array}$ \\
\hline \multirow{3}{*}{ não tratado } & linear & 32 & 7.141 & 1.000 & & & \\
\hline & quadrático & 31 & 6.568 & 1.000 & 0.573 & 1 & 0.449 \\
\hline & cúbico & 30 & 5.324 & 1.000 & 1.817 & 1 & 0.178 \\
\hline \multirow{3}{*}{ tratado } & linear & 34 & 16.311 & 0.996 & & & \\
\hline & quadrático & 33 & 16.178 & 0.994 & 0.133 & 1 & 0.715 \\
\hline & cúbico & 32 & 15.866 & 0.992 & 0.445 & 1 & 0.505 \\
\hline
\end{tabular}


Tabela 3.3.1b: Ajustes dos modelos logísticos para os dois grupos, tratado e não tratado segundo a estatística de Hosmer e Lemeshow.

\begin{tabular}{|c|c|c|c|c|}
\hline Grupo & Modelo & g.1. & $\begin{array}{c}\text { Estatística de Hosmer e } \\
\text { Lemeshow }\end{array}$ & $\begin{array}{c}\text { Nível } \\
\text { descritivo }\end{array}$ \\
\hline \multirow{3}{*}{ tratado } & linear & 9 & 1,217 & 0,999 \\
\hline & quadrático & 9 & 1,079 & 0,999 \\
\hline & cúbico & 9 & 0,788 & 1,000 \\
\hline \multirow{3}{*}{ não tratado } & linear & 10 & 2,223 & 0,994 \\
\hline & quadrático & 10 & 2,710 & 0,987 \\
\hline & cúbico & 10 & 6,057 & 0,810 \\
\hline
\end{tabular}

As Tabelas 3.3.1a e 3.3.1b indicam que todos os modelos parecem se ajustar adequadamente aos dados, tanto para o grupo tratado quanto para o não tratado, mas o modelo linear é o mais adequado nos dois casos, de acordo com as estatísticas da razão de verossimilhança apresentadas na Tabela3.3.1a. As Tabelas 3.3.2 e 3.3.3 contém as estimativas dos parâmetros do modelo ajustado para cada grupo.

Tabela 3.3.2: Estimativas dos parâmetros do modelo linear para o grupo não tratado.

\begin{tabular}{lccc}
\hline Coeficientes & Estimativa & $\begin{array}{c}\text { Desvio } \\
\text { padrão }\end{array}$ & $\begin{array}{c}\text { Valor da } \\
\text { estatistica Z }\end{array}$ \\
\hline intercepto & $-1,390$ & 0,344 & $-4,042$ \\
coeficiente linear & $-0,051$ & 0,016 & $-3,181$ \\
\hline
\end{tabular}

Tabela 3.3.3: Estimativas dos parâmetros do modelo linear para o grupo tratado.

\begin{tabular}{lccc}
\hline \multicolumn{1}{c}{ Coeficientes } & Estimativa & $\begin{array}{c}\text { Desvio } \\
\text { padrão }\end{array}$ & $\begin{array}{c}\text { Valor da } \\
\text { estatistica Z }\end{array}$ \\
\hline intercepto & $-2,661$ & 0,302 & $-8,793$ \\
coeficiente linear & $-0,033$ & 0,010 & $-3,176$ \\
\hline
\end{tabular}


As Tabelas 3.3 .4 e 3.3 .5 contém as estimativas da taxa de risco e função de sobrevivência e seus respectivos desvios-padrão aproximados para alguns dos intervalos segundo o modelo linear para os grupos tratado e não tratado.

Tabela 3.3.4: Estimativas da taxa de risco e função de sobrevivência e seus respectivos desvios-padrão aproximados para alguns dos intervalos no modelo linear para o grupo não tratado.

\begin{tabular}{|c|c|c|c|c|}
\hline Mês & $\begin{array}{c}\text { Taxa de } \\
\text { risco }\end{array}$ & $\begin{array}{l}\text { Desvio } \\
\text { padrão }\end{array}$ & $\begin{array}{c}\text { Função de } \\
\text { sobrevivência }\end{array}$ & $\begin{array}{l}\text { Desvio } \\
\text { padrão }\end{array}$ \\
\hline 5 & 0,179 & 0,047 & 0,820 & 0,046 \\
\hline 10 & 0,145 & 0,033 & 0,702 & 0,067 \\
\hline 15 & 0,116 & 0,025 & 0,620 & 0,075 \\
\hline 20 & 0,092 & 0,020 & 0,563 & 0,078 \\
\hline 25 & 0,073 & 0,018 & 0,523 & 0,079 \\
\hline 30 & 0,057 & 0,017 & 0,493 & 0,079 \\
\hline 35 & 0,045 & 0,016 & 0,471 & 0,080 \\
\hline 40 & 0,035 & 0,014 & 0,454 & 0,080 \\
\hline 45 & 0,027 & 0,013 & 0,442 & 0,080 \\
\hline 50 & 0,021 & 0,012 & 0,432 & 0,081 \\
\hline 60 & 0,013 & 0,009 & 0,420 & 0,082 \\
\hline 70 & 0,008 & 0,006 & 0,412 & 0,084 \\
\hline 80 & 0,004 & 0,004 & 0,408 & 0,085 \\
\hline 100 & 0,002 & 0,002 & 0,404 & 0,086 \\
\hline 120 & $<0,001$ & $<0,001$ & 0,403 & 0,087 \\
\hline 140 & $<0,001$ & $<0,001$ & 0,402 & 0,087 \\
\hline
\end{tabular}

Note que para os pacientes não tratados, o risco de morte por câncer no ovário é maior no início do período que sucede a segunda laparotomia e decresce ao longo do tempo. Para o grupo tratado o comportamento do risco de morte é o mesmo, mas comparado com o grupo não tratado, esse risco parece ser sempre menor, consequentemente a probabilidade de sobrevida no grupo tratado é maior que no grupo não tratado. Esse fato pode ser visualizado 
pelos Gráficos 3.3.3 e 3.3.4 que contêm as curvas ajustadas de cada tratamento tanto para a taxa de risco quanto para a função de sobrevivência. Para complementar, os Gráficos 3.3.5 e 3.3.6 mostram as curvas referentes às taxas de risco e as bandas de confiança de $95 \%$ em cada grupo separadamente. Analogamente, os Gráficos 3.3.7 e 3.3.8 mostram as curvas de sobrevida e as bandas de confiança de $95 \%$ também para cada grupo.

Tabela 3.3.5: Estimativas da taxa de risco e função de sobrevivência e seus respectivos desvios-padrão aproximados para alguns dos intervalos no modelo linear para o grupo tratado.

\begin{tabular}{|c|c|c|c|c|}
\hline Mês & $\begin{array}{c}\text { Taxa de } \\
\text { risco }\end{array}$ & $\begin{array}{l}\text { Desvio } \\
\text { padrão }\end{array}$ & $\begin{array}{c}\text { Função de } \\
\text { sobrevivência }\end{array}$ & $\begin{array}{l}\text { Desvio } \\
\text { padrão }\end{array}$ \\
\hline 5 & 0,061 & 0,016 & 0,939 & 0,016 \\
\hline 10 & 0,052 & 0,012 & 0,890 & 0,027 \\
\hline 15 & 0,044 & 0,010 & 0,851 & 0,034 \\
\hline 20 & 0,038 & 0,008 & 0,818 & 0,038 \\
\hline 25 & 0,032 & 0,007 & 0,792 & 0,042 \\
\hline 30 & 0,028 & 0,006 & 0,770 & 0,044 \\
\hline 35 & 0,024 & 0,005 & 0,752 & 0,046 \\
\hline 40 & 0,020 & 0,005 & 0,736 & 0,047 \\
\hline 45 & 0,017 & 0,005 & 0,724 & 0,048 \\
\hline 50 & 0,015 & 0,005 & 0,713 & 0,049 \\
\hline 60 & 0,011 & 0,004 & 0,697 & 0,051 \\
\hline 70 & 0,008 & 0,004 & 0,685 & 0,053 \\
\hline 80 & 0,005 & 0,003 & 0,677 & 0,054 \\
\hline 100 & 0,003 & 0,002 & 0,667 & 0,057 \\
\hline 120 & 0,001 & 0,001 & 0,662 & 0,059 \\
\hline 140 & $<0,001$ & $<0,001$ & 0,659 & 0,061 \\
\hline
\end{tabular}

Para estabelecer uma relação quantitativa entre as chances de morte no dois grupos, considerou-se uma medida equivalente à razão das chances em alguns intervalos de interesse. 
Por exemplo, na ocasião do quinto mês, que pelo modelo é o intervalo onde o risco de morte é maior, a chance de morte no grupo não tratado é 3,257 vezes maior do que no grupo tratado. Essa desvantagem do grupo não tratado diminui ao longo do tempo, com 10 meses vale 2,977, com 40 meses 1,734 e assim segue. Isso pode significar que os pacientes não tratados que conseguirem sobreviver aos primeiros meses tendem a ter a mesma chance de morte que os tratados a medida em que os anos passam.

Apesar da estatística do desvio ter indicado um ótimo ajuste tanto para o grupo tratado quanto para o não tratado é sempre adequado fazer uma análise de resíduos para tentar detectar possíveis problemas do modelo adotado.

Com relação ao grupo não tratado os Gráficos 3.3.9, 3.3.10 e 3.3.11 não indicam a existência de pontos aberrantes ou influentes, e nem mesmo de desvios das suposições do modelo. Da mesma forma, no grupo tratado, os Gráficos 3.3.12 e 3.3.14 indicam que não há pontos aberrantes e que o modelo está bem ajustado. Entretanto, O Gráfico 3.3.13 da distância de Cook pelos valores ajustados das probabilidades no grupo tratado, destaca o ponto referente ao primeiro intervalo que, quando presente nos dados, altera a estimativa do coeficiente linear em $27 \%$. A influência desse ponto pode ser explicada pelo fato de apresentar uma proporção baixa de eventos, relativamente aos intervalos próximos. 
Gráfico 3.3.1: Taxa de risco observada para o grupo não tratado.

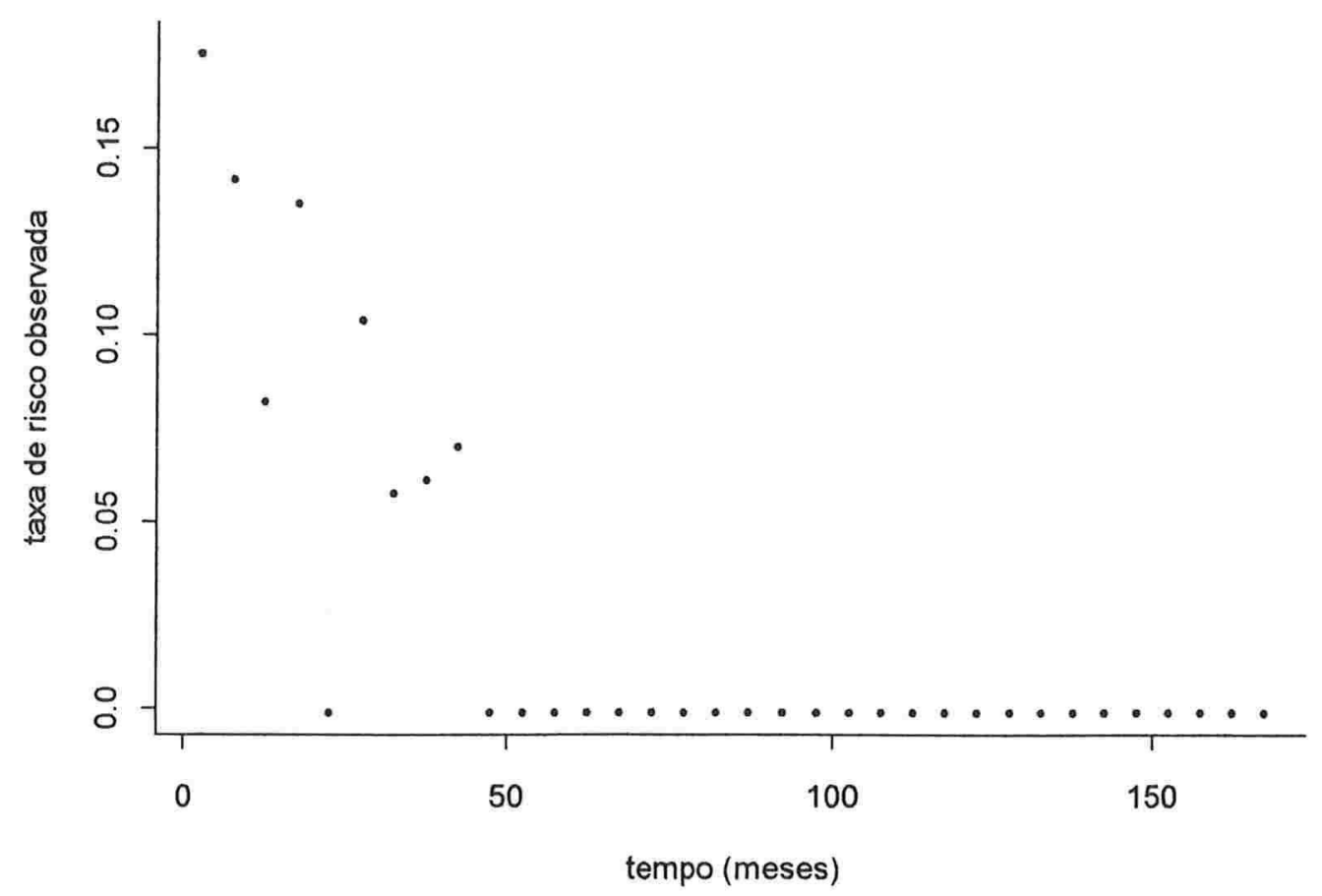

Gráfico 3.3.2: Taxas de risco observada para o grupo tratado.

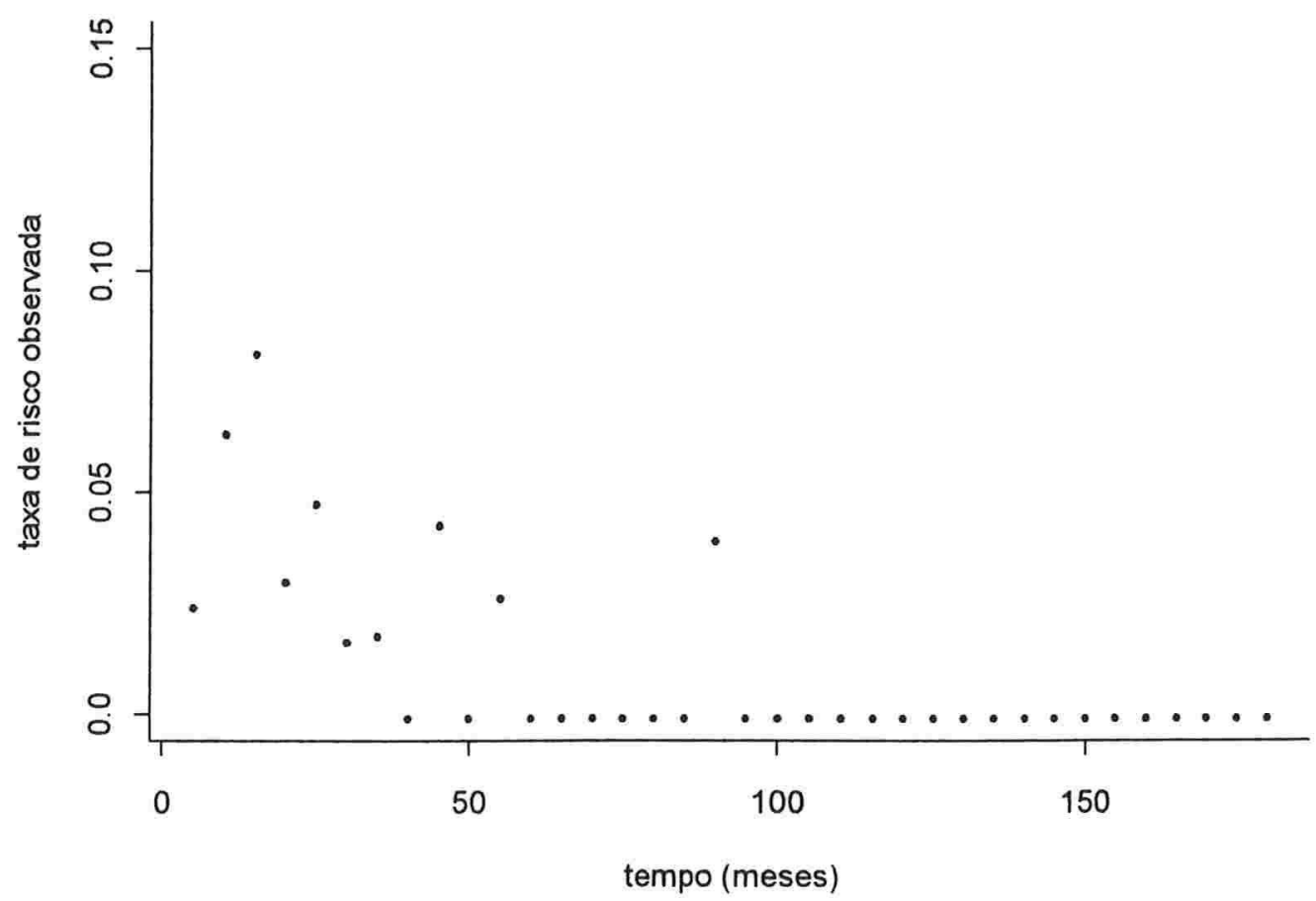


Gráfico 3.3.3: Taxas de risco estimadas para os grupos tratado e não tratado.

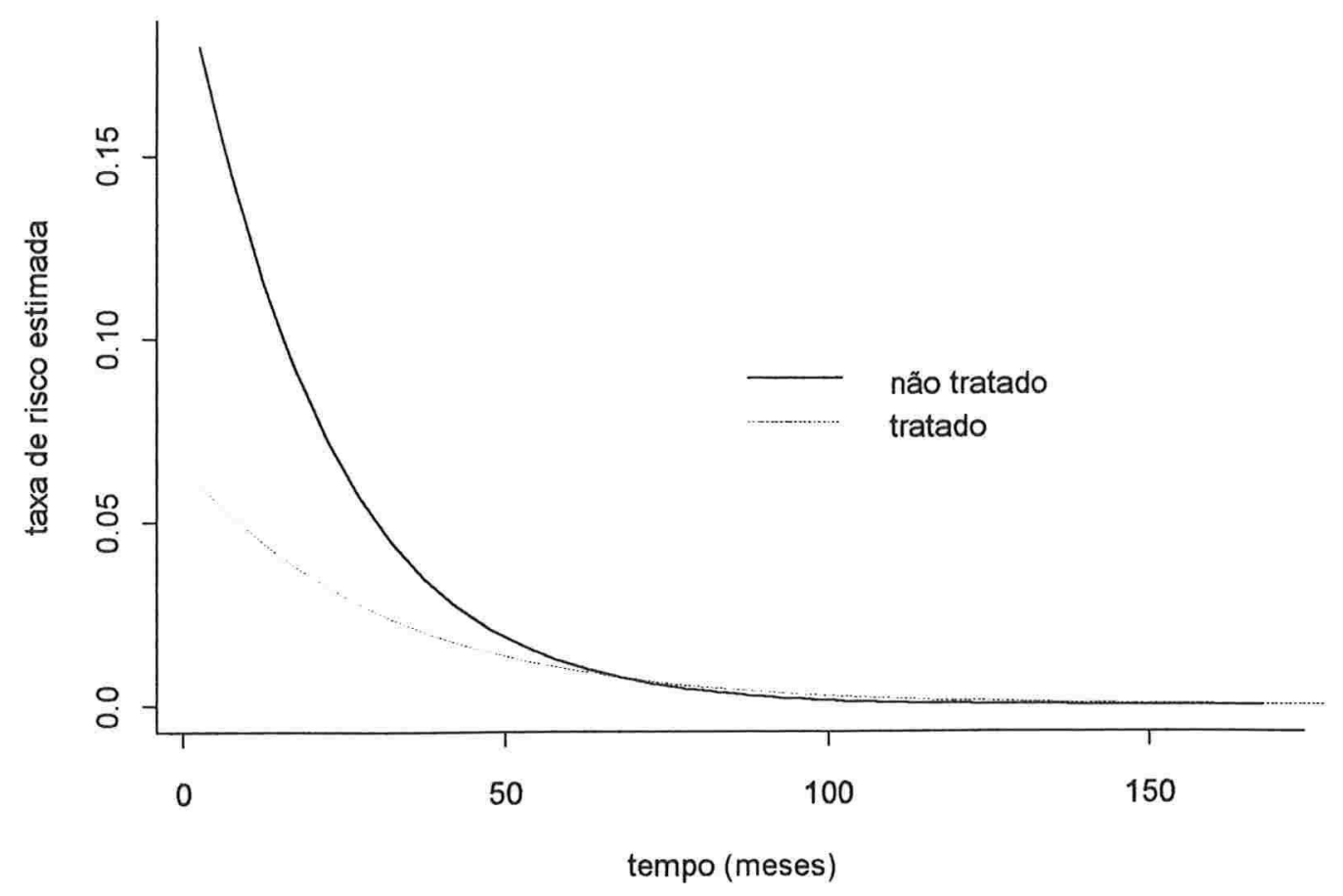

Gráfico 3.3.4: Curvas de sobrevida estimadas para os grupos tratado e não tratado.

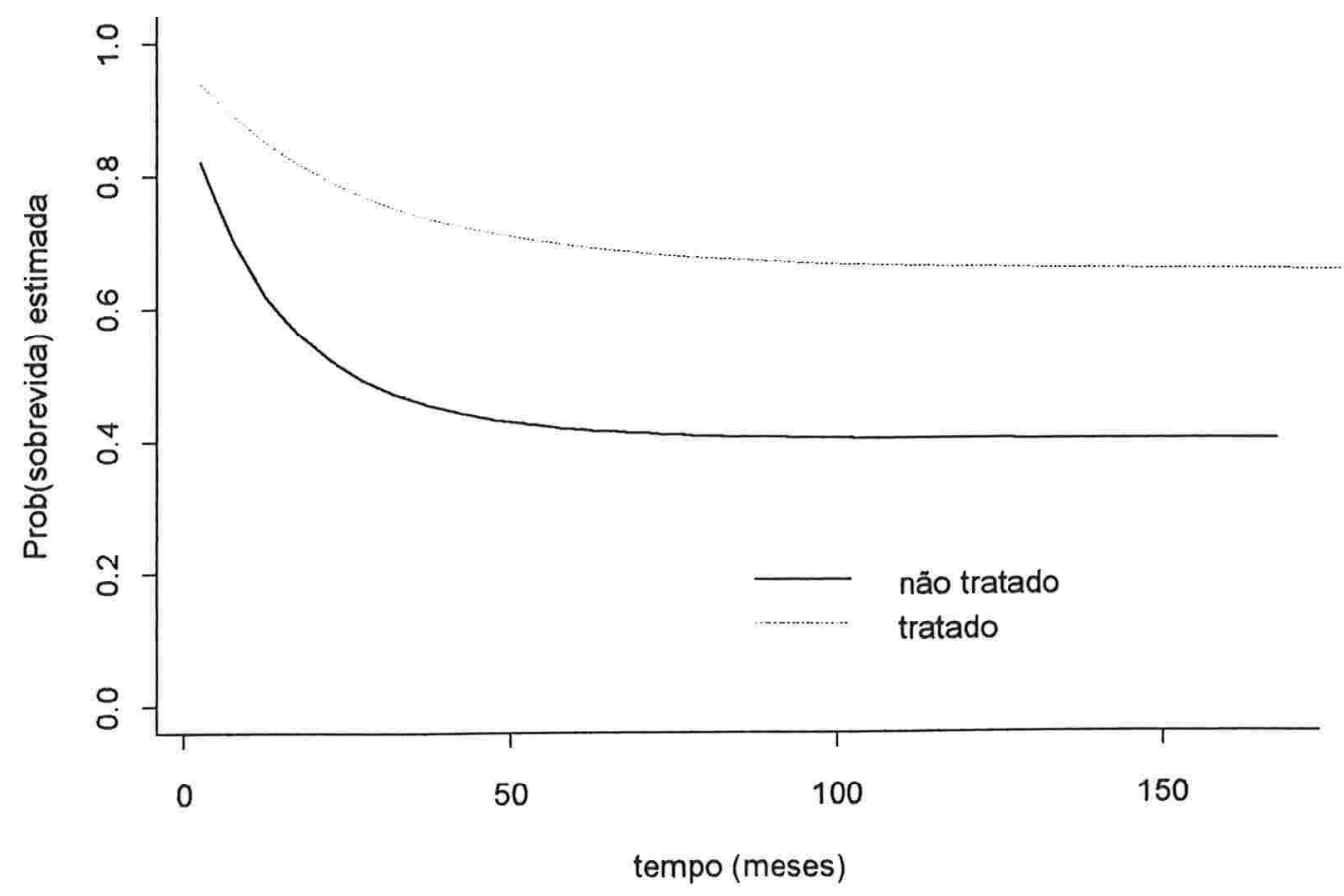


Gráfico 3.3.5: Taxa de risco e bandas de confiança (95\%) estimadas para o grupo não tratado.

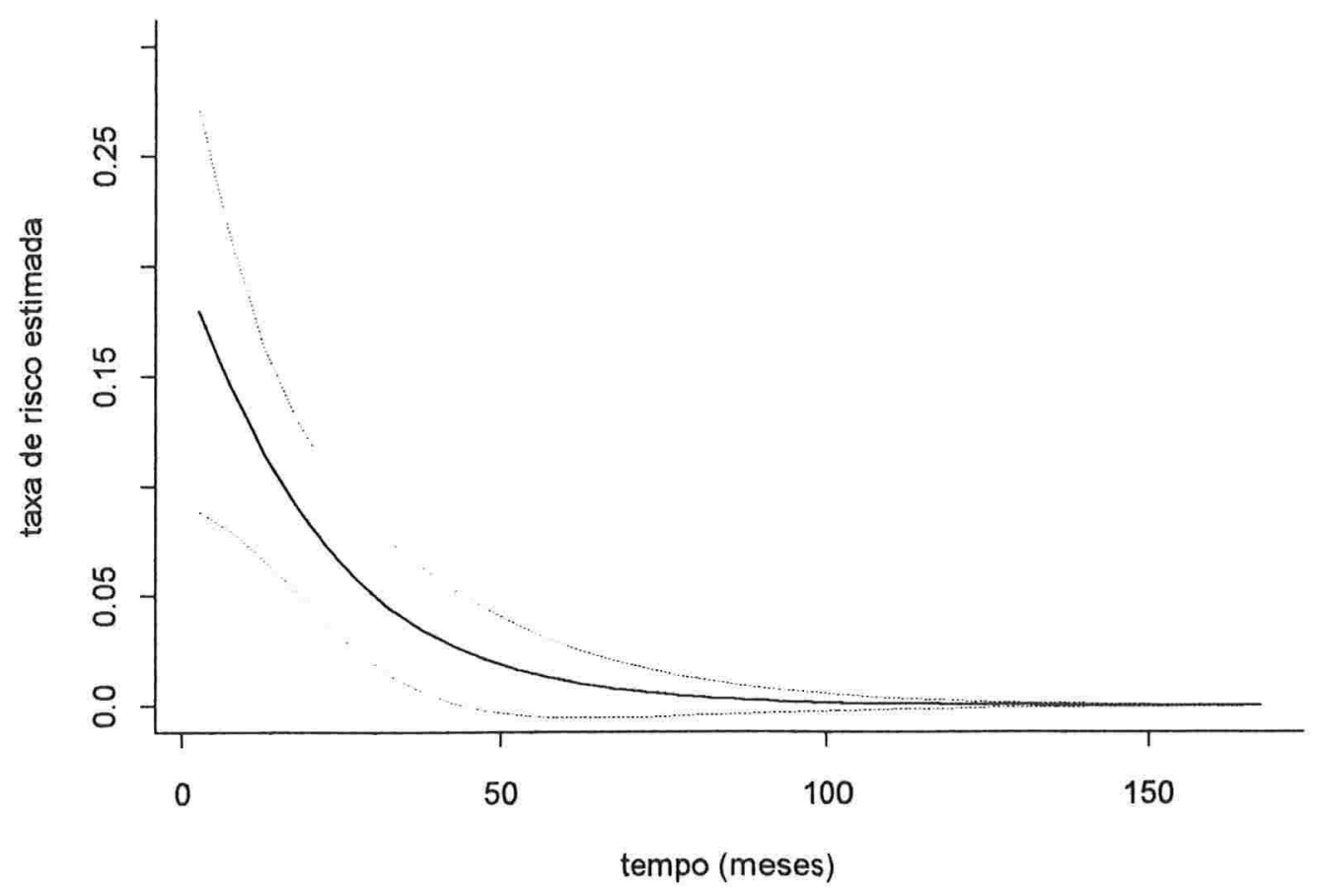

Gráfico 3.3.6: Taxa de risco e bandas de confiança (95\%) estimadas para o grupo tratado.

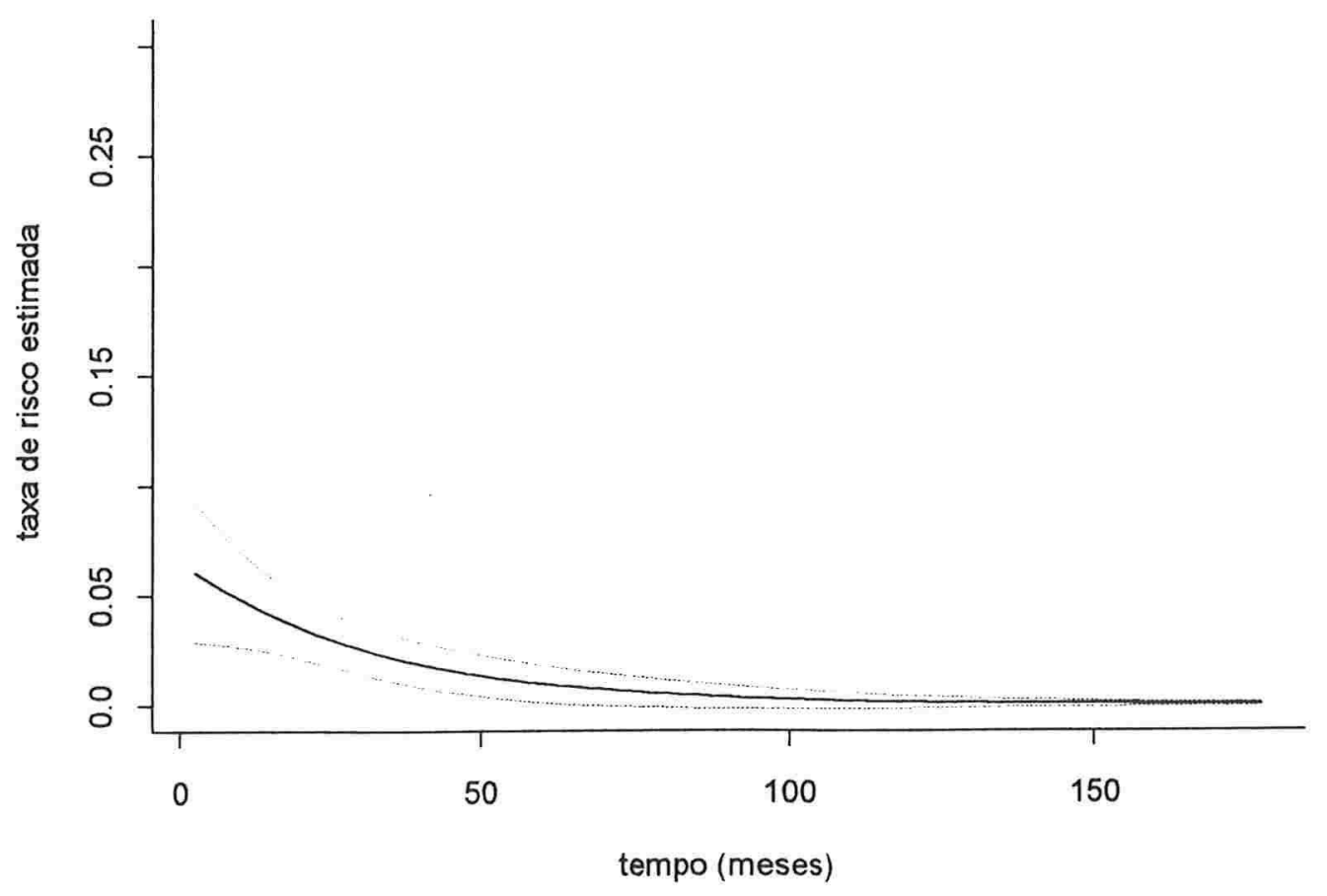


Gráfico 3.3.7: Curva de sobrevida e bandas de confiança (95\%) estimadas para os não tratados.

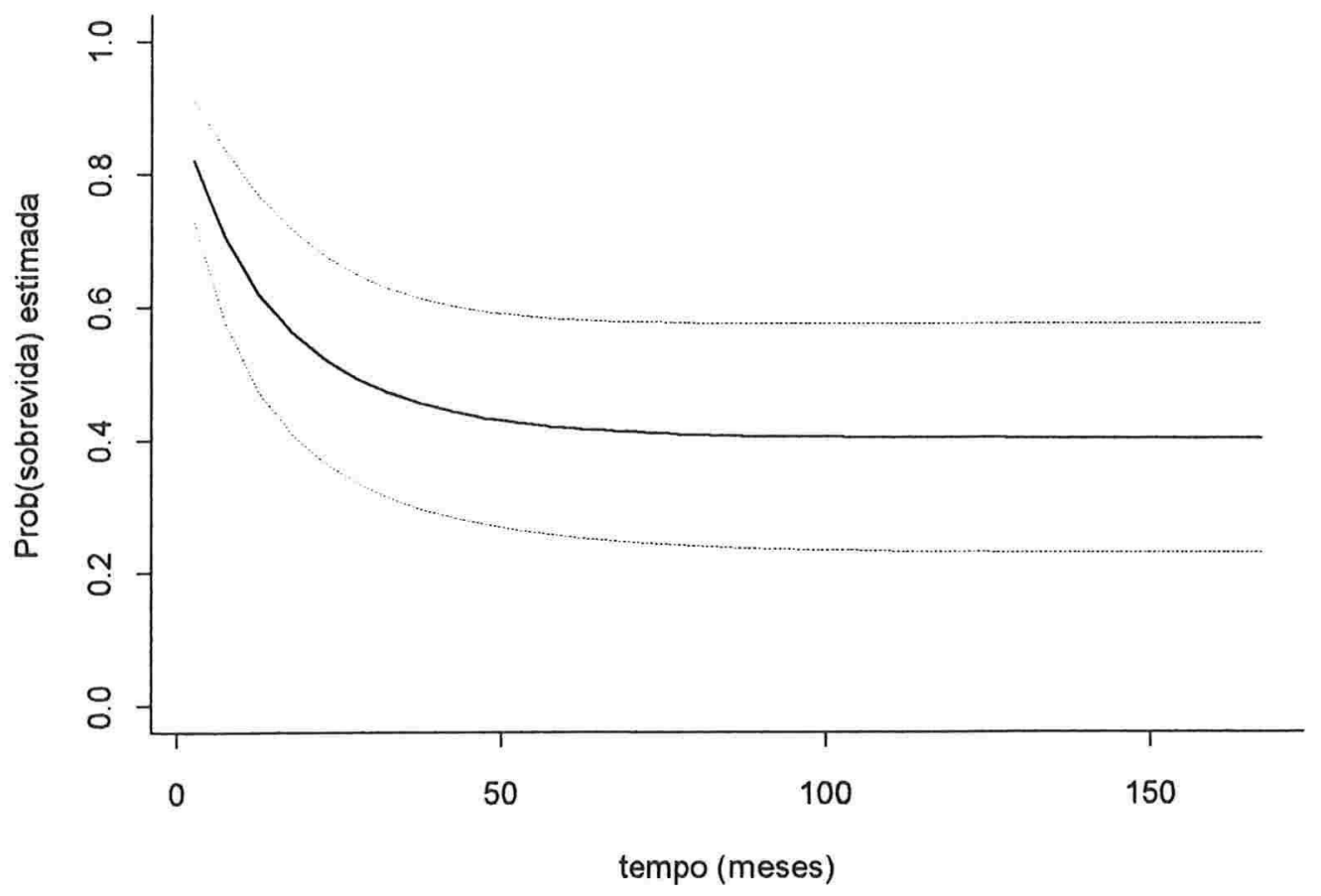

Gráfico 3.3.8: Curva de sobrevida e bandas de confiança (95\%) estimadas para os tratados.

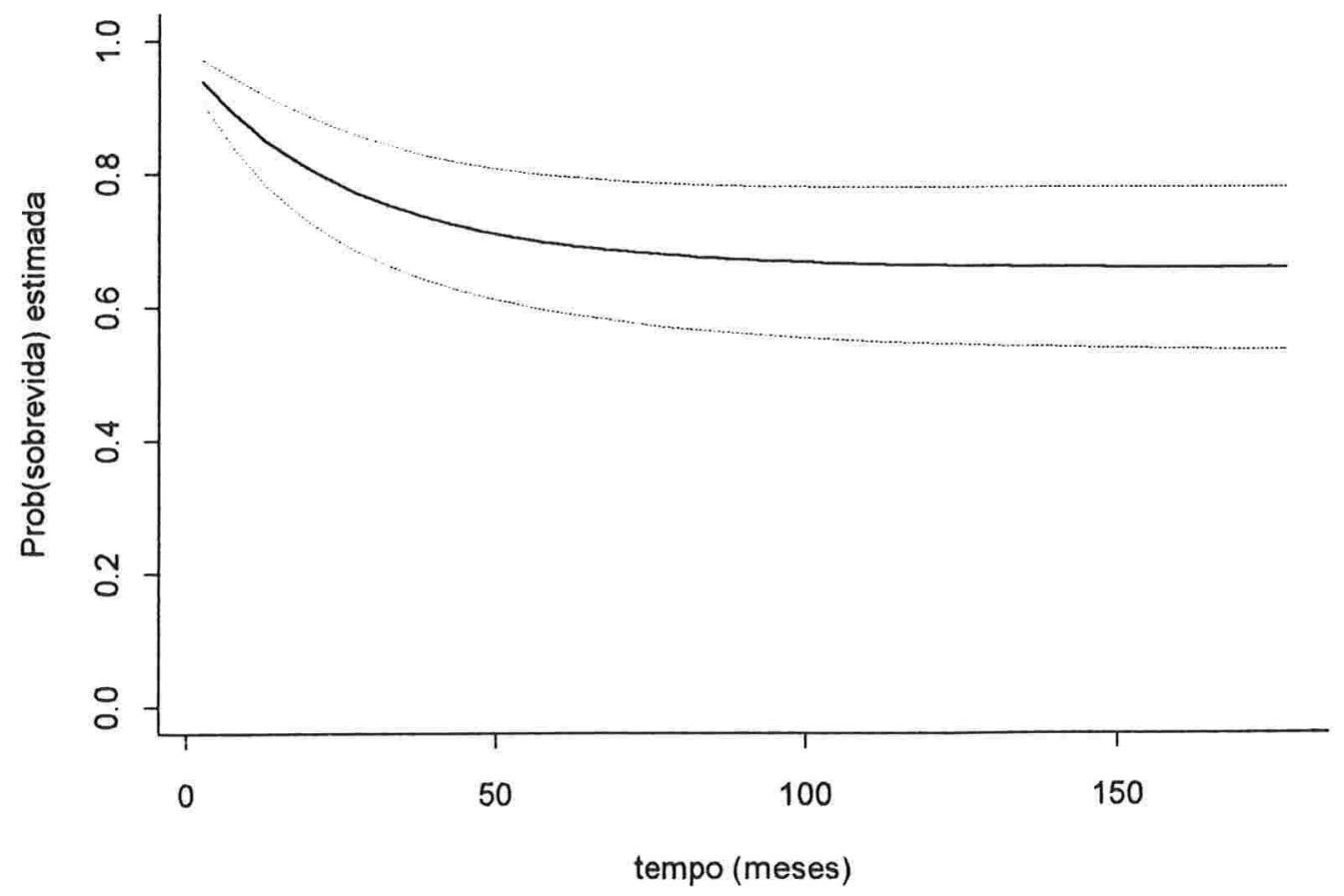


Gráfico 3.3.9: Gráfico do $t_{D_{i}}$ para detectar pontos aberrantes no grupo não tratado.

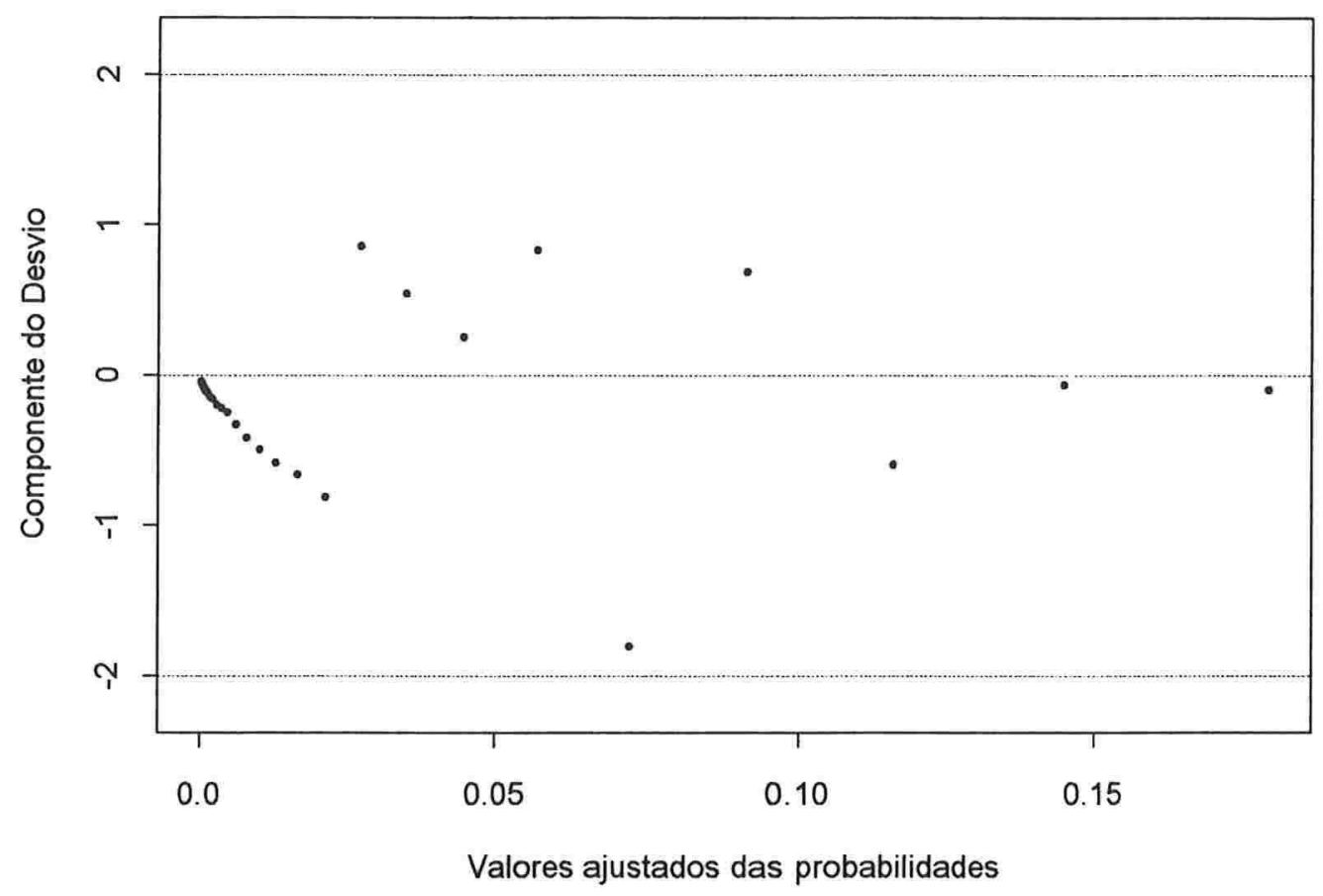

Gráfico 3.3.10: Gráfico do $L D_{i}$ para detectar pontos influentes no grupo não tratado.

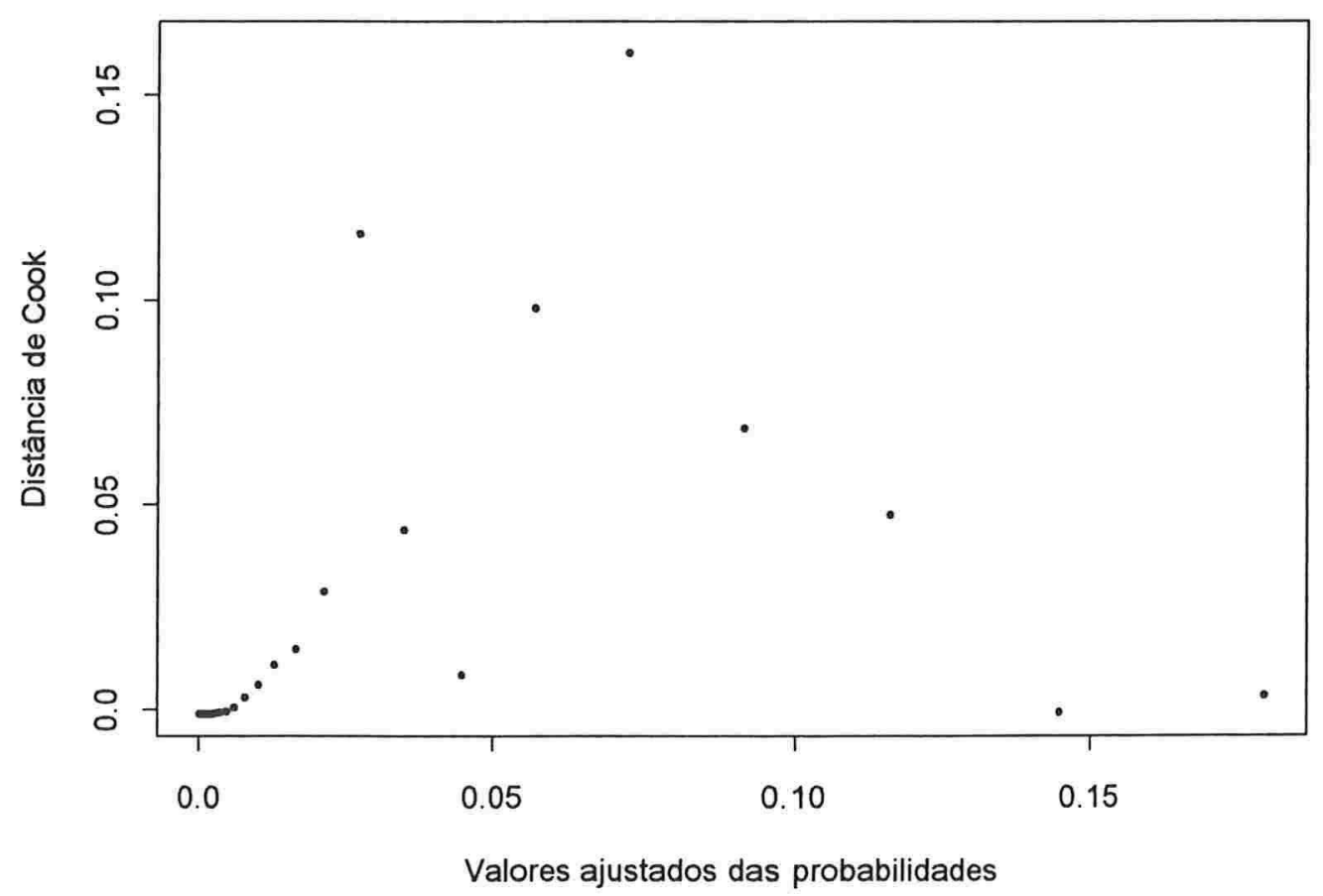


Gráfico 3.3.11: Resíduos padronizados e envelopes para o grupo não tratado.

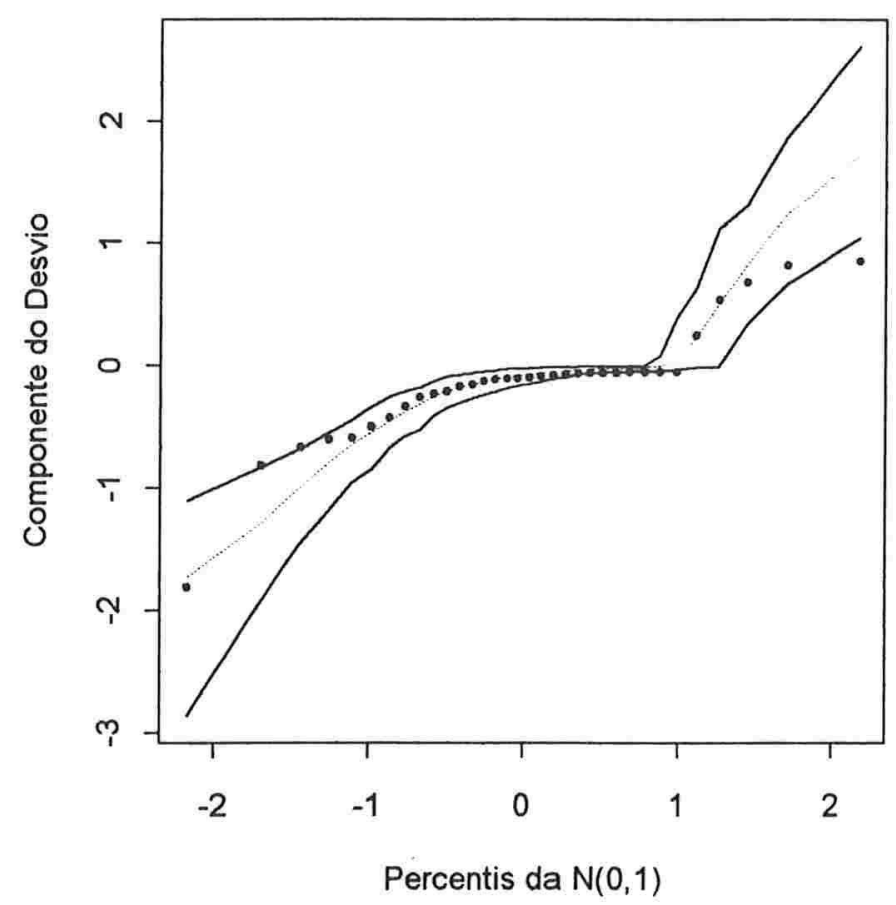

Gráfico 3.3.12: Gráfico do $t_{D_{1}}$ para detectar pontos aberrantes no grupo tratado.

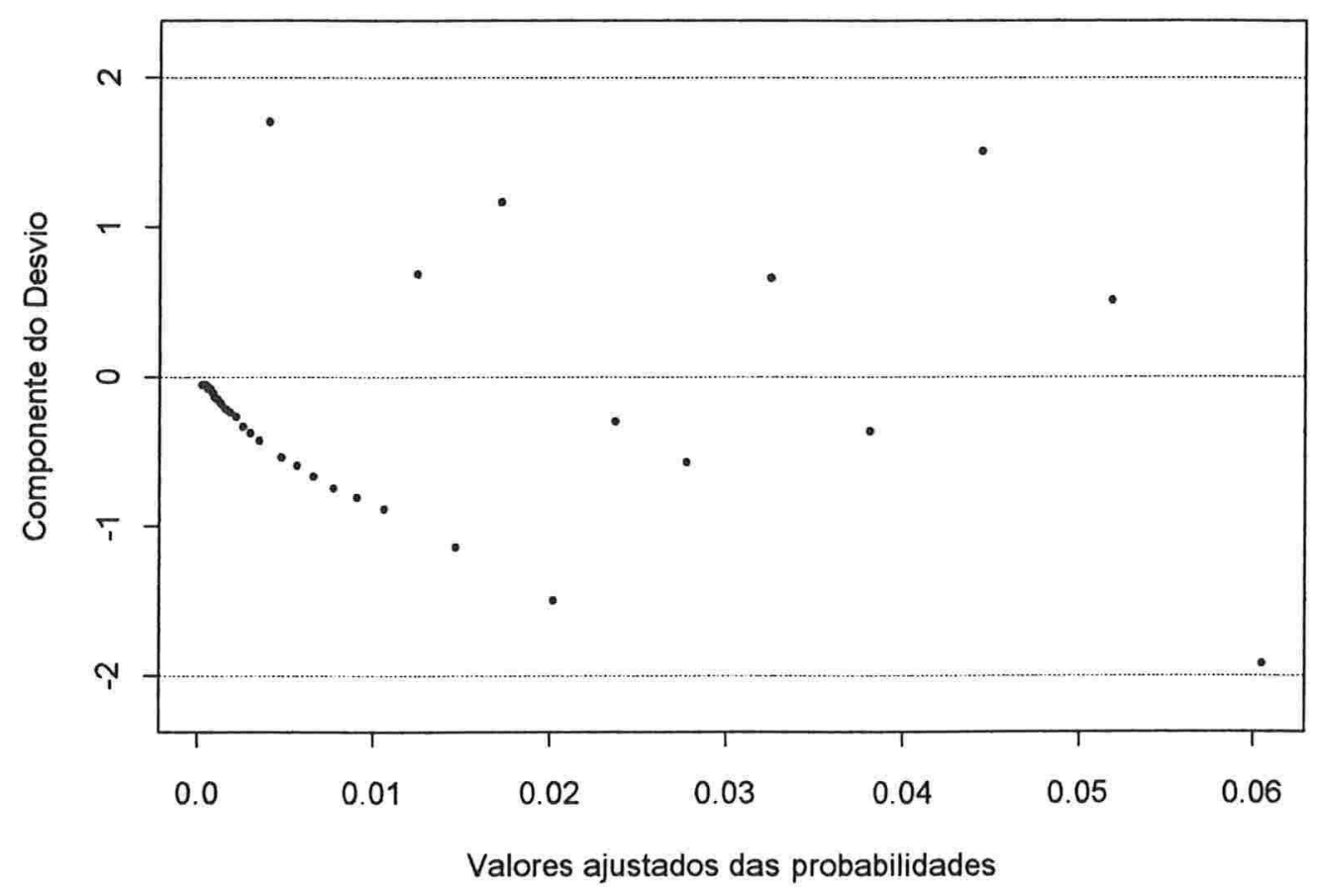


Gráfico 3.3.13: Gráfico do $L D_{i}$ para detectar pontos influentes no grupo tratado.

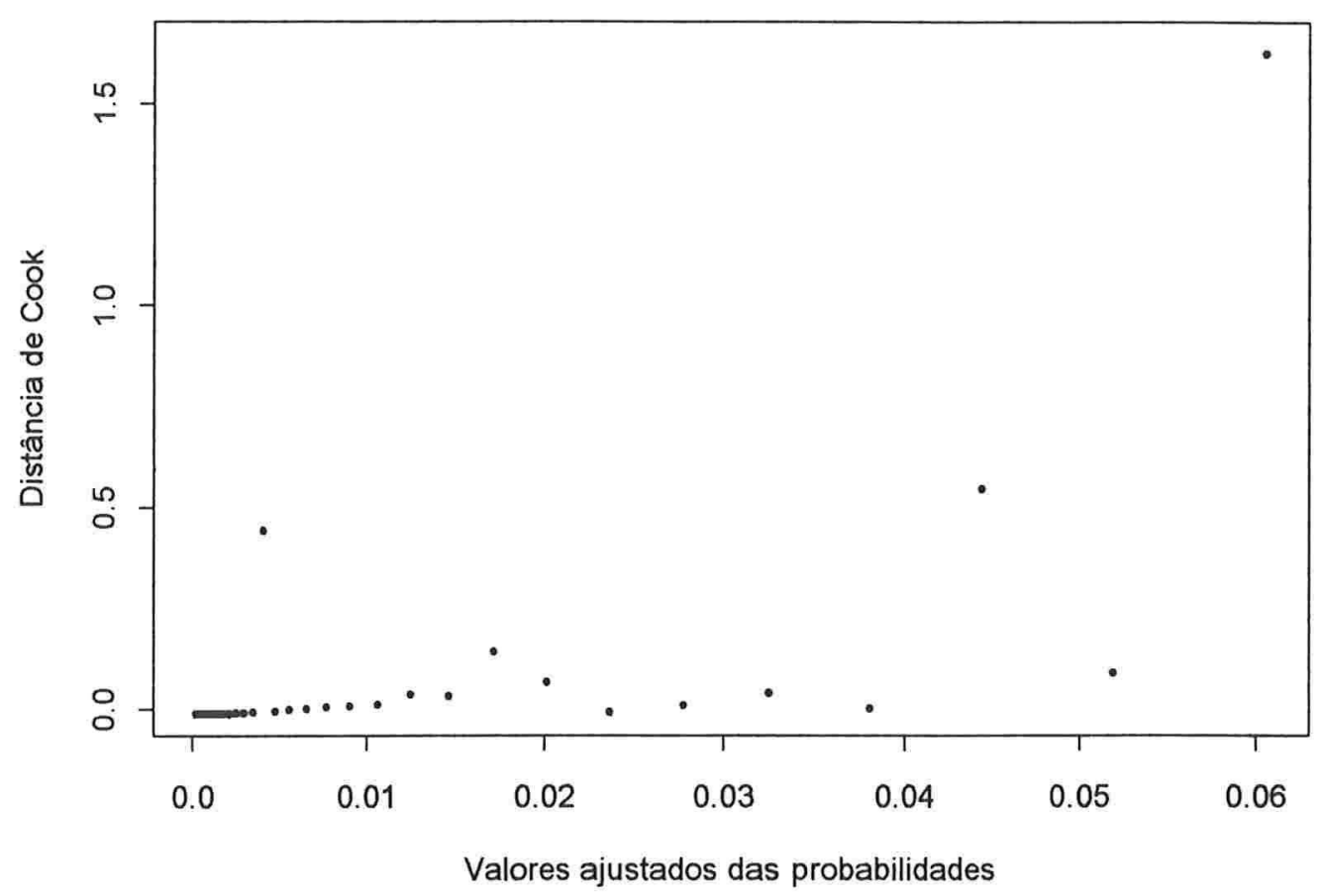

Gráfico 3.3.14: Resíduos padronizados e envelopes para o grupo tratado.

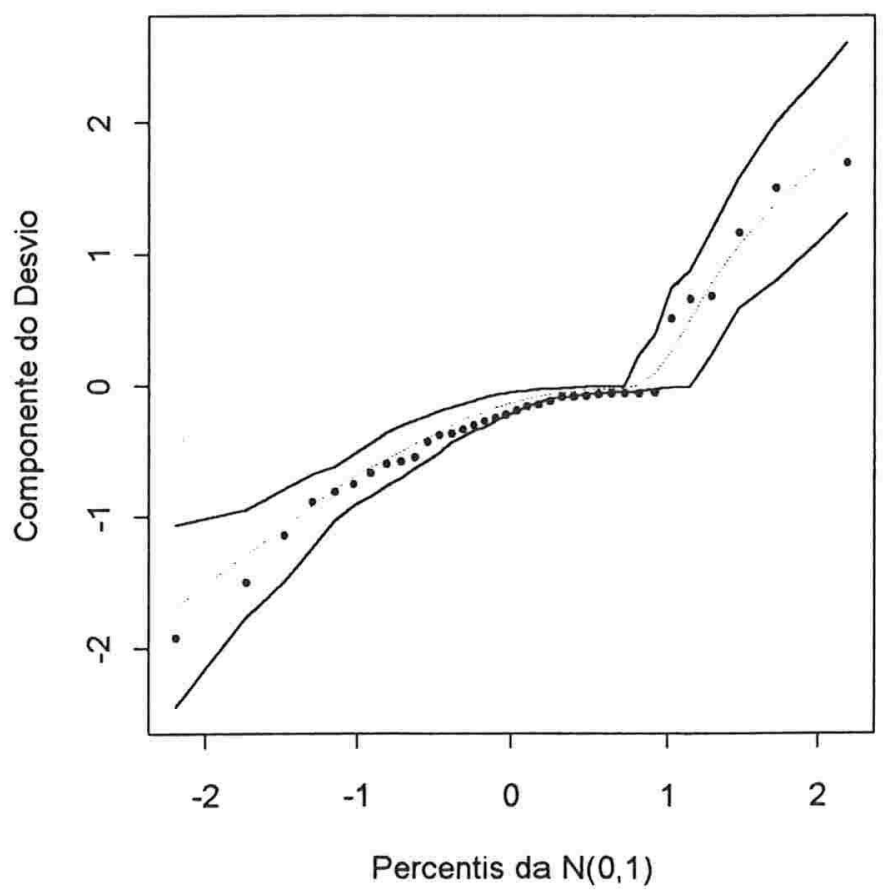




\section{CONCLUSÃO}

Nesse trabalho procurou-se mostrar uma técnica paramétrica para análise de dados de sobrevivência através da regressão logística, recomendada para problemas em que existe interesse em estudar o comportamento da função de risco ao longo do tempo. A Regressão Logística Parcial é uma alternativa à técnica de estimação não-paramétrica de Kaplan-Meier, que, ao contrário de outras técnicas paramétricas clássicas, não impõe uma forma fixa para a função de risco, permitindo assim que a sua variação seja modelada.

A extensão para mais de dois tratamentos é direta já que não é necessário ajustar o mesmo modelo para todos os tratamentos, embora isso facilite a comparação, mesmo que empírica, das curvas obtidas. Portanto, se não houver interesse em estabelecer comparações entre tratamentos, modelos diferentes podem ser considerados.

Procurou-se apresentar, adicionalmente, um conjunto de técnicas de diagnóstico e de avaliação da qualidade do ajuste, úteis na escolha do melhor modelo. Tais procedimentos integram uma etapa importante da análise, uma vez que fornecem informações quanto a afastamentos importantes das suposições feitas para o modelo adotado, que em alguns casos podem ser reparados.

A técnica de Regressão Logística Parcial pode ser, portanto, muito útil na análise de dados de sobrevivência, além de apresentar algumas vantagens sobre as técnicas paramétricas usuais, tais como a possibilidade de modelagem da função de risco e uso de técnicas desenvolvidas para os modelos lineares generalizados, uma vez que o modelo logístico faz parte dessa classe. 
APENDICE A

DADOS 
Dados referente ao Exemplo 1.1 - Dados hipotéticos

$\begin{array}{lll}\text { tratamento } & \text { tempo } & \text { censura } \\ 1 & 3 & 1 \\ 1 & 5 & 1 \\ 1 & 7 & 1 \\ 1 & 9 & 0 \\ 1 & 13 & 1 \\ 1 & 22 & 1 \\ 1 & 25 & 1 \\ 1 & 35 & 0 \\ 2 & 2 & 1 \\ 2 & 6 & 1 \\ 2 & 10 & 1 \\ 2 & 12 & 0 \\ 2 & 20 & 0 \\ 2 & 23 & 1 \\ 2 & 33 & 1 \\ 2 & 34 & 1\end{array}$




\section{Dados referente ao exemplo 1.2}

trata- meses dias censura mento

$\begin{array}{llll}1 & 1 & 7 & 1 \\ 1 & 2 & 34 & 1 \\ 1 & 2 & 42 & 1 \\ 1 & 3 & 63 & 1 \\ 1 & 3 & 64 & 1 \\ 1 & 3 & 83 & 1 \\ 1 & 3 & 84 & 1 \\ 1 & 3 & 91 & 1 \\ 1 & 3 & 74 & 0 \\ 1 & 4 & 108 & 1 \\ 1 & 4 & 112 & 1 \\ 1 & 5 & 129 & 1 \\ 1 & 5 & 133 & 1 \\ 1 & 5 & 133 & 1 \\ 1 & 5 & 139 & 1 \\ 1 & 5 & 140 & 1 \\ 1 & 5 & 140 & 1 \\ 1 & 5 & 146 & 1 \\ 1 & 5 & 149 & 1 \\ 1 & 6 & 154 & 1 \\ 1 & 6 & 157 & 1 \\ 1 & 6 & 160 & 1 \\ 1 & 6 & 160 & 1 \\ 1 & 6 & 165 & 1 \\ 1 & 6 & 173 & 1 \\ 1 & 6 & 176 & 1 \\ 1 & 7 & 185 & 0 \\ 1 & 8 & 218 & 1 \\ 1 & 8 & 225 & 1 \\ 1 & 8 & 241 & 1 \\ 1 & 9 & 248 & 1 \\ 1 & 9 & 273 & 1\end{array}$
trata- meses dias censura
mento

$\begin{array}{llll}1 & 10 & 277 & 1 \\ 1 & 10 & 297 & 1 \\ 1 & 10 & 279 & 0 \\ 1 & 11 & 319 & 0 \\ 1 & 14 & 405 & 1 \\ 1 & 14 & 417 & 1 \\ 1 & 14 & 420 & 1 \\ 1 & 15 & 440 & 1 \\ 1 & 18 & 523 & 1 \\ 1 & 18 & 523 & 0 \\ 1 & 20 & 583 & 1 \\ 1 & 20 & 594 & 1 \\ 1 & 37 & 1101 & 1 \\ 1 & 37 & 1116 & 0 \\ 1 & 38 & 1146 & 1 \\ 1 & 41 & 1226 & 0 \\ 1 & 45 & 1349 & 0 \\ 1 & 47 & 1417 & 1 \\ 1 & 47 & 1412 & 0 \\ 2 & 1.5 & 37 & 1 \\ 2 & 3 & 84 & 1 \\ 2 & 3.5 & 92 & 1 \\ 2 & 3.5 & 94 & 1 \\ 2 & 4 & 110 & 1 \\ 2 & 4 & 112 & 1 \\ 2 & 4 & 119 & 1 \\ 2 & 4.5 & 127 & 1 \\ 2 & 4.5 & 130 & 1 \\ 2 & 4.5 & 133 & 1 \\ 2 & 5 & 140 & 1 \\ 2 & 5 & 146 & 1 \\ 2 & 5.5 & 155 & 1\end{array}$
trata- meses dias censura
mento

$\begin{array}{llll}2 & 5.5 & 159 & 1 \\ 2 & 6 & 173 & 1 \\ 2 & 6 & 179 & 1 \\ 2 & 6 & 169 & 0 \\ 2 & 6.5 & 194 & 1 \\ 2 & 6.5 & 195 & 1 \\ 2 & 7 & 209 & 1 \\ 2 & 8.5 & 249 & 1 \\ 2 & 10 & 281 & 1 \\ 2 & 11 & 319 & 1 \\ 2 & 12 & 339 & 1 \\ 2 & 15 & 432 & 1 \\ 2 & 16 & 469 & 1 \\ 2 & 18 & 519 & 1 \\ 2 & 18 & 528 & 0 \\ 2 & 18 & 547 & 0 \\ 2 & 21 & 633 & 1 \\ 2 & 21 & 613 & 0 \\ 2 & 24 & 725 & 1 \\ 2 & 25 & 759 & 0 \\ 2 & 27 & 817 & 1 \\ 2 & 37 & 1092 & 0 \\ 2 & 41 & 1245 & 0 \\ 2 & 45 & 1331 & 0 \\ 2 & 53 & 1557 & 1 \\ 2 & 55 & 1642 & 0 \\ 2 & 59 & 1776 & 1 \\ 2 & 59 & 1771 & 0 \\ 2 & 63 & 1897 & 0 \\ 2 & 67 & 2023 & 0 \\ 2 & 71 & 2146 & 0 \\ 2 & 77 & 2297 & 0\end{array}$

Fonte: Efron, B. (1988). Logistic Regression, Survival Analysis, and the Kaplan-Meier Curve. Journal of the American Statistical Association, 83, 414-425. 
Dados referente ao exemplo 2.1

\begin{tabular}{|c|c|c|c|c|c|c|c|c|c|c|c|}
\hline $\mathrm{ni}$ & si & mês & $\begin{array}{l}\text { trata- } \\
\text { mento }\end{array}$ & ni & si & mês & $\begin{array}{l}\text { trata- } \\
\text { mento }\end{array}$ & ni & si & mês & $\begin{array}{l}\text { trata- } \\
\text { mento }\end{array}$ \\
\hline 51 & 1 & 1 & 1 & 7 & 1 & 37 & 1 & 19 & 0 & 17 & 2 \\
\hline 50 & 2 & 2 & 1 & 5 & 1 & 38 & 1 & 19 & 1 & 18 & 2 \\
\hline 48 & 5 & 3 & 1 & 4 & 0 & 39 & 1 & 16 & 0 & 19 & 2 \\
\hline 42 & 2 & 4 & 1 & 4 & 0 & 40 & 1 & 16 & 0 & 20 & 2 \\
\hline 40 & 8 & 5 & 1 & 4 & 0 & 41 & 1 & 16 & 1 & 21 & 2 \\
\hline 32 & 7 & 6 & 1 & 3 & 0 & 42 & 1 & 14 & 0 & 22 & 2 \\
\hline 25 & 0 & 7 & 1 & 3 & 0 & 43 & 1 & 14 & 0 & 23 & 2 \\
\hline 24 & 3 & 8 & 1 & 3 & 0 & 44 & 1 & 14 & 1 & 24 & 2 \\
\hline 21 & 2 & 9 & 1 & 3 & 0 & 45 & 1 & 13 & 0 & 25 & 2 \\
\hline 19 & 2 & 10 & 1 & 2 & 0 & 46 & 1 & 12 & 0 & 26 & 2 \\
\hline 16 & 0 & 11 & 1 & 2 & 1 & 47 & 1 & 12 & 1 & 27 & 2 \\
\hline 15 & 0 & 12 & 1 & 45 & 0 & 0.5 & 2 & 11 & 0 & 29 & 2 \\
\hline 15 & 0 & 13 & 1 & 45 & 0 & 1 & 2 & 11 & 0 & 31 & 2 \\
\hline 15 & 3 & 14 & 1 & 45 & 1 & 1.5 & 2 & 11 & 0 & 33 & 2 \\
\hline 12 & 1 & 15 & 1 & 44 & 0 & 2 & 2 & 11 & 0 & 35 & 2 \\
\hline 11 & 0 & 16 & 1 & 44 & 0 & 2.5 & 2 & 11 & 0 & 37 & 2 \\
\hline 11 & 0 & 17 & 1 & 44 & 1 & 3 & 2 & 10 & 0 & 39 & 2 \\
\hline 11 & 1 & 18 & 1 & 43 & 2 & 3.5 & 2 & 10 & 0 & 41 & 2 \\
\hline 9 & 0 & 19 & 1 & 41 & 3 & 4 & 2 & 9 & 0 & 43 & 2 \\
\hline 9 & 2 & 20 & 1 & 38 & 3 & 4.5 & 2 & 9 & 0 & 45 & 2 \\
\hline 7 & 0 & 21 & 1 & 35 & 2 & 5 & 2 & 8 & 0 & 47 & 2 \\
\hline 7 & 0 & 22 & 1 & 33 & 2 & 5.5 & 2 & 8 & 0 & 49 & 2 \\
\hline 7 & 0 & 23 & 1 & 31 & 2 & 6 & 2 & 8 & 0 & 51 & 2 \\
\hline 7 & 0 & 24 & 1 & 28 & 2 & 6.5 & 2 & 8 & 1 & 53 & 2 \\
\hline 7 & 0 & 25 & 1 & 26 & 1 & 7 & 2 & 7 & 0 & 55 & 2 \\
\hline 7 & 0 & 26 & 1 & 25 & 0 & 7.5 & 2 & 6 & 0 & 57 & 2 \\
\hline 7 & 0 & 27 & 1 & 25 & 0 & 8 & 2 & 6 & 1 & 59 & 2 \\
\hline 7 & 0 & 28 & 1 & 25 & 1 & 8.5 & 2 & 4 & 0 & 61 & 2 \\
\hline 7 & 0 & 29 & 1 & 24 & 0 & 9 & 2 & 4 & 0 & 63 & 2 \\
\hline 7 & 0 & 30 & 1 & 24 & 1 & 10 & 2 & 3 & 0 & 65 & 2 \\
\hline 7 & 0 & 31 & 1 & 23 & 1 & 11 & 2 & 3 & 0 & 67 & 2 \\
\hline 7 & 0 & 32 & 1 & 22 & 1 & 12 & 2 & 2 & 0 & 69 & 2 \\
\hline 7 & 0 & 33 & 1 & 21 & 0 & 13 & 2 & 2 & 0 & 71 & 2 \\
\hline 7 & 0 & 34 & 1 & 21 & 0 & 14 & 2 & 1 & 0 & 73 & 2 \\
\hline 7 & 0 & 35 & 1 & 21 & 1 & 15 & 2 & 1 & 0 & 75 & 2 \\
\hline 7 & 0 & 36 & 1 & 20 & 1 & 16 & 2 & 1 & 0 & 77 & 2 \\
\hline
\end{tabular}

Fonte: Efron, B. (1988). Logistic Regression, Survival Analysis, and the Kaplan-Meier Curve. Journal of the American Statistical Association, 83, 414-425. 
Dados referente ao exemplo 3.1

$\begin{array}{lrr}\text { ano } & \text { ni } & \text { si } \\ 1 & & \\ 2 & 3941 & 456 \\ 3 & 3485 & 384 \\ 4 & 3101 & 359 \\ 5 & 2742 & 295 \\ 6 & 2447 & 218 \\ 7 & 2229 & 184 \\ 8 & 2045 & 123 \\ 9 & 1642 & 79 \\ 10 & 1256 & 53 \\ 11 & 948 & 35 \\ 12 & 648 & 16 \\ & 391 & 5\end{array}$

Fonte: Singer J. D. e Willett J. B. (1995). It's about time: Using Discrete-time Survival Analysis to Study Duration and the Timing Events. The Journal of Educational Statistics. 
Dados referente ao exemplo 3.2

$\begin{array}{cccccccc}\text { dias } & \text { ni } & \text { si } & \text { massa } & \text { dias } & \text { ni } & \text { si } & \text { massa } \\ 5 & 92 & 0 & 0 & 140 & 2 & 0 & 0 \\ 10 & 92 & 0 & 0 & 5 & 112 & 0 & 1 \\ 15 & 91 & 0 & 0 & 10 & 112 & 0 & 1 \\ 20 & 88 & 0 & 0 & 15 & 110 & 0 & 1 \\ 25 & 86 & 2 & 0 & 20 & 108 & 0 & 1 \\ 30 & 73 & 1 & 0 & 25 & 107 & 0 & 1 \\ 35 & 66 & 3 & 0 & 30 & 101 & 1 & 1 \\ 40 & 61 & 1 & 0 & 35 & 92 & 1 & 1 \\ 45 & 54 & 0 & 0 & 40 & 82 & 1 & 1 \\ 50 & 46 & 5 & 0 & 45 & 74 & 0 & 1 \\ 55 & 39 & 0 & 0 & 50 & 66 & 2 & 1 \\ 60 & 35 & 0 & 0 & 55 & 59 & 0 & 1 \\ 65 & 33 & 1 & 0 & 60 & 48 & 2 & 1 \\ 70 & 28 & 1 & 0 & 65 & 37 & 2 & 1 \\ 75 & 25 & 0 & 0 & 70 & 32 & 4 & 1 \\ 80 & 21 & 3 & 0 & 75 & 24 & 0 & 1 \\ 85 & 16 & 2 & 0 & 80 & 19 & 1 & 1 \\ 90 & 11 & 1 & 0 & 85 & 14 & 3 & 1 \\ 95 & 9 & 0 & 0 & 90 & 8 & 0 & 1 \\ 100 & 9 & 2 & 0 & 95 & 8 & 0 & 1 \\ 105 & 7 & 1 & 0 & 100 & 6 & 0 & 1 \\ 110 & 4 & 0 & 0 & 105 & 5 & 1 & 1 \\ 115 & 4 & 0 & 0 & 110 & 4 & 2 & 1 \\ 120 & 3 & 0 & 0 & 115 & 2 & 0 & 1 \\ 125 & 3 & 0 & 0 & 120 & 1 & 0 & 1 \\ 130 & 2 & 0 & 0 & 125 & 1 & 0 & 1 \\ 135 & 2 & 0 & 0 & 130 & 1 & 0 & 1 \\ & & & & 135 & 1 & 0 & 1\end{array}$

Fonte: Paula, G. A., Barbosa, L. S. e Ferreira, R. F. G. (1989). Relatório de Análise Estatística sobre o Projeto: Comportamento biológico evolutivo do tumor KB no decorrer de suas passagens seriadas em ratos nudes adultos. RAE-SEA-8904, IME-USP 
Dados referente ao exemplo 3.3

\begin{tabular}{|c|c|c|c|c|c|c|c|}
\hline mes & $\mathrm{ni}$ & si & tratamento & mes & ni & si & tratamento \\
\hline 5 & 34 & 6 & 0 & 10 & 78 & 5 & 1 \\
\hline 10 & 28 & 4 & 0 & 15 & 73 & 6 & 1 \\
\hline 15 & 24 & 2 & 0 & 20 & 65 & 2 & 1 \\
\hline 20 & 22 & 3 & 0 & 25 & 62 & 3 & 1 \\
\hline 25 & 19 & 0 & 0 & 30 & 58 & 1 & 1 \\
\hline 30 & 19 & 2 & 0 & 35 & 54 & 1 & 1 \\
\hline 35 & 17 & 1 & 0 & 40 & 49 & 0 & 1 \\
\hline 40 & 16 & 1 & 0 & 45 & 46 & 2 & 1 \\
\hline 45 & 14 & 1 & 0 & 50 & 39 & 0 & 1 \\
\hline 50 & 13 & 0 & 0 & 55 & 37 & 1 & 1 \\
\hline 55 & 11 & 0 & 0 & 60 & 32 & 0 & 1 \\
\hline 60 & 11 & 0 & 0 & 65 & 31 & 0 & 1 \\
\hline 65 & 10 & 0 & 0 & 70 & 31 & 0 & 1 \\
\hline 70 & 9 & 0 & 0 & 75 & 29 & 0 & 1 \\
\hline 75 & 7 & 0 & 0 & 80 & 27 & 0 & 1 \\
\hline 80 & 5 & 0 & 0 & 85 & 26 & 0 & 1 \\
\hline 85 & 5 & 0 & 0 & 90 & 25 & 1 & 1 \\
\hline 90 & 5 & 0 & 0 & 95 & 22 & 0 & 1 \\
\hline 95 & 4 & 0 & 0 & 100 & 20 & 0 & 1 \\
\hline 100 & 4 & 0 & 0 & 105 & 18 & 0 & 1 \\
\hline 105 & 3 & 0 & 0 & 110 & 13 & 0 & 1 \\
\hline 110 & 3 & 0 & 0 & 115 & 12 & 0 & 1 \\
\hline 115 & 3 & 0 & 0 & 120 & 11 & 0 & 1 \\
\hline 120 & 3 & 0 & 0 & 125 & 9 & 0 & 1 \\
\hline 125 & 2 & 0 & 0 & 130 & 7 & 0 & 1 \\
\hline 130 & 2 & 0 & 0 & 135 & 6 & 0 & 1 \\
\hline 135 & 1 & 0 & 0 & 140 & 4 & 0 & 1 \\
\hline 140 & 1 & 0 & 0 & 145 & 2 & 0 & 1 \\
\hline 145 & 1 & 0 & 0 & 150 & 2 & 0 & 1 \\
\hline 150 & 1 & 0 & 0 & 155 & 2 & 0 & 1 \\
\hline 155 & 1 & 0 & 0 & 160 & 1 & 0 & 1 \\
\hline 160 & 1 & 0 & 0 & 165 & 1 & 0 & 1 \\
\hline 165 & 1 & 0 & 0 & 170 & 1 & 0 & 1 \\
\hline 170 & 1 & 0 & 0 & 175 & 1 & 0 & 1 \\
\hline 5 & 80 & 2 & 1 & 180 & 1 & 0 & 1 \\
\hline
\end{tabular}

Fonte: Lima, A. C. P. (1996). MAE783-Análise de sobrevivência. Curso ministrado no Instituto de Matemática e Estatística da USP, São Paulo. 
APÊNDICE B

\section{PROGRAMAS NO SPLUS}




\section{Programa referente ao exemplo 1.1}

dados<-scan("c:Ilcarinellteselldados $\mid l e x e 1 \_1 . t x t "$, what $=$ list(trat $=0$, tempo $=0$, censura $\left.=0\right)$ ) attach (dados)

\#\#\#\#\#\# ajuste da curva de Kaplan-Meier e teste de Mantel-Haenszel \#\#\#\#\#\#\#

fitl<-surv.fit(tempo,censura, strata=trat,type="kaplan-meier",error="greenwood", conf.level=0.95) surv.diff(tempo,censura, trat, rho $=0$ )

\#\#\#\#\#\# representação gráfica da curva de Kaplan-Meier \#\#\#\#\#\#\#

win.printer(file="c:Ilcarinelltesellgraficollgraphl_1.wmf", format ="placeable metafile")

plot.surv.fit(fit1 ,xlab="meses", ylab="proporção de sobreviventes",lty=c( $(1,2), \mathrm{cex}=1.09)$

legend $(18,0.95, c($ "Tratamento 1 ", "Tratamento $2 "), 1$ ty $=c(1,2)$, bty=" $n ", c e x=1.09)$

dev.off()

\section{Programa referente ao exemplo 1.2}

dados $2<-$ scan("c:Ilcarinellteselldados $\mid$ dados $2 . t x t "$, what $=\operatorname{list}($ trat $=0$, meses $=0$, dias $=0$, censura $=0)$ ) attach (dados2)

\#\#\#\#\#\# ajuste da curva de Kaplan-Meier e teste de Mantel-Haenszel \#\#\#\#\#\#\#

fit $1<-$ surv.fit(meses,censura, strata=trat,type="kaplan-meier",error="greenwood", conf.level=0.95) surv.diff(dias, censura, trat, rho $=0$ )

\#\#\#\#\#\# representação gráfica da curva de Kaplan-Meier \#\#\#\#\#\#\#

win.printer(file="c:Ilcarinelltesellgraficollgraphl_2. wmf", format ="placeable metafile") plot.surv.fit(fit1 ,xlab="meses", ylab="proporção de sobreviventes",1ty=c(1,2), cex=1.09) legend(40,0.8,c("Tratamento A", "Tratamento B"),lty=c(1,2), bty="n", cex=1.09) dev.off()

\section{Programa referente ao exemplo 2.1}

dados1<-scan("c:Ilcarinellteselldados $\mid$ dadosl.txt", what=list(ni=0, si =0, mes $=0$, trat $=0)$ ) attach (dados1)

\#\#\#\#\#\# Preparação dos dados para utilização da técnica de regressão logística parcial

$\mathrm{y}<-\mathrm{si}$

$\mathrm{n}<-\mathrm{ni}$

$\mathrm{yA}<-0$

$\mathrm{nA}<-0$

$\operatorname{mes} A<-0$

$\mathrm{yB}<-0$

$\mathrm{nB}<-0$

mesB $<-0$

\#\#\#\#\#\# divisao dos vetores segundo os tratamentos A e B

for(i in 1:47)

\{

$y A[i]<-y[i]$

$n A[i]<-n[i]$

$\operatorname{mes} \mathrm{A}[\mathrm{i}]<-\operatorname{mes}[\mathrm{i}]\}$ 
for(i in $1: 61)$

\{

$y B[i]<-y[i+47]$

$\mathrm{nB}[\mathrm{i}]<-\mathrm{n}[\mathrm{i}+47]$

$\operatorname{mesB}[\mathrm{i}]<-\operatorname{mes}[\mathrm{i}+47]\}$

\#\#\#\#\#\# Gráfico da taxa de risco observada para obter uma idéia do seu comportamento

hiA obs $<-y \mathrm{~A} / \mathrm{nA}$

win.printer(file="c:Ilcarinelltesellgraficollgraph2_A.wmf", format ="placeable metafile")

plot(mesA,hiA_obs, bty="l", xlab="tempo meses",ylab="taxa de risco observada", cex=1.09)

dev.off()

hiB_obs<-yB/nB

win.printer(file="c:Ilcarinelltesellgraficollgraph2_B.wmf", format ="placeable metafile")

plot(mesB,hiB_obs, bty="l", xlab="tempo meses",ylab="taxa de risco observada", cex=1.09)

dev.off()

Regressao Logistica Parcial

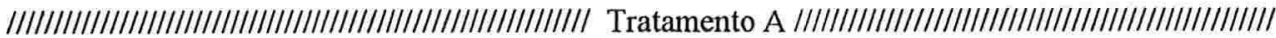

ymatA $<-$ cbind $(\mathrm{yA}, \mathrm{nA}-\mathrm{yA})$

freqA $<-y A / n A$

mesA<-mesA-0.5

\#\#\#\#\# Modelo linear para o tratamento A

fit $1 \mathrm{~A}<-\operatorname{glm}(\mathrm{ymat} A \sim \operatorname{mes} \mathrm{A}$, family=binomial $)$

vetor $1 \mathrm{~A}<-\operatorname{coef}($ fitl $\mathrm{A})$

summary(fit1A)

\#\#\#\#\# Modelo cúbico para o tratamento A

fit $2 \mathrm{~A}<-\operatorname{glm}\left(\mathrm{ymat} A \sim \operatorname{mes} \mathrm{A}+\operatorname{mes}^{\wedge} 2+\operatorname{mes}^{\wedge} 3\right.$, family=binomial $)$

vetor $2 \mathrm{~A}<-\operatorname{coef}($ fit $2 \mathrm{~A})$

summary(fit2A)

\#\#\#\#\# Modelo cubico-linear "spline" para o tratamento A

$\times 2<-0$

for ( $\mathrm{i}$ in $1: 47$ )

if $(\operatorname{mes} A[\mathrm{i}]<11) \times 2[\mathrm{i}]<-(\operatorname{mes} A[\mathrm{i}]-11)^{\wedge} 2$ else $\times 2[\mathrm{i}]<-0$

$\mathrm{x} 3<-0$

for ( $\mathrm{i}$ in 1:47)

if $(\operatorname{mes} A[\mathrm{i}]<11) \times 3[\mathrm{i}]<-(\operatorname{mes} A[\mathrm{i}]-11)^{\wedge} 3$ else $\times 3[\mathrm{i}]<-0$

fit $3 A<-g l m(y m a t A \sim \operatorname{mes} A+x 2+x 3$, family=binomial)

vetor $3 A<-\operatorname{coef}($ fit $3 A)$

summary(fit $3 \mathrm{~A}$ )

Modelo escolhido para o tratamento A : cubico-linear "spline" 
\#\#\#\#\# calculo do risco

$\mathrm{x}<-$ vetor $3 \mathrm{~A}[1]$-vetor $3 \mathrm{~A}[2]^{*}$ mesA-vetor $3 \mathrm{~A}[3]^{*} \times 2$-vetor $3 \mathrm{~A}[4]^{*} \mathrm{x} 3$

$\mathrm{x}<-\exp (\mathrm{x})$

$\mathrm{h} 3 \mathrm{~A}<-1 /(1+\mathrm{x})$

\#\#\#\#\# cálculo do desvio-padrão da função de risco e intervalo de confiança de $95 \%$

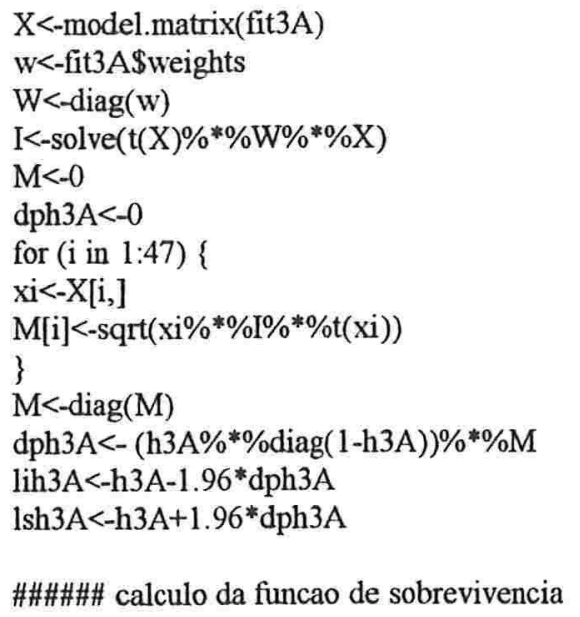

$\mathrm{h}<-1-\mathrm{h} 3 \mathrm{~A}$

G3A<-cumprod(h)

\#\#\#\#\# calculo do desvio da função de sobrevivência e intervalo de confiança de $95 \%$

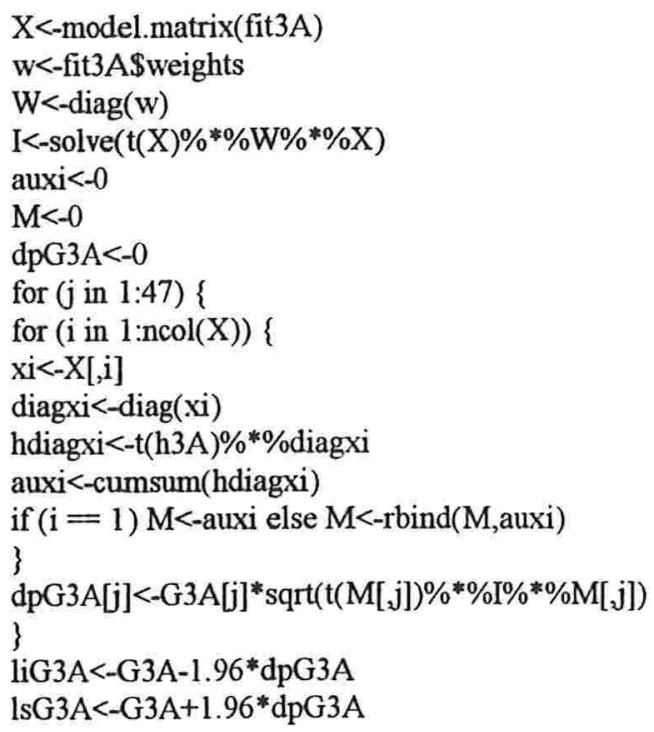

Ajuste do modelo de superdispersão para o tratamento A:

fit $4 A<-g \operatorname{lm}(y m a t A \sim m e s A+x 2+x 3$, family=quasi $($ link=logit , variance="mu( $(1-m u) "))$ 
offsett $<-0$

for $(\mathrm{i}$ in $1: 18)\{$

mesB[i]<-mesB[i]-0.25

offsett $[\mathrm{i}]<-\log (0.5)\}$

for ( $\mathrm{i}$ in 1:18) \{

mesB[i+18]<-mesB[i+18]-0.5

offsett $[\mathrm{i}+18]<-0\}$

for (i in 1:25)

$\operatorname{mesB}[i+36]<-\operatorname{mes} B[i+36]-1$

offsett $[\mathrm{i}+36]<-\log (2)\}$

ymatB <- cbind $(y B, n B-y B)$

\#\#\#\#\# Modelo linear para o tratamento B

fit1B <- glm(ymatB mesB+offset(offsett), family=binomial)

vetor $1 \mathrm{~B}<-$ coef(fit1B)

summary(fit1B)

\#\#\#\#\# Modelo cúbico para o tratamento B

fit $2 \mathrm{~B}<-\operatorname{glm}\left(\mathrm{ymat} B \sim \operatorname{mes} \mathrm{B}+\operatorname{mes}^{\wedge} 2+\operatorname{mesB}^{\wedge} 3+\right.$ offset(offsett), family=binomial)

vetor $2 \mathrm{~B}<-\operatorname{coef}($ fit2B)

summary(fit2B)

\#\#\#\#\# Modelo cubico-linear "spline" para o tratamento B

$\mathrm{x} 4<-0$

for ( $\mathrm{i}$ in 1:61)

if $(\operatorname{mesB}[\mathrm{i}]<11) \times 4[\mathrm{i}]<-(\operatorname{mesB}[\mathrm{i}]-11)^{\wedge} 2$ else $\times 4[\mathrm{i}]<-0$

$\times 5<-0$

for (i in 1:61)

if $(\operatorname{mesB}[\mathrm{i}]<11) \times 5[\mathrm{i}]<-(\operatorname{mesB}[\mathrm{i}]-11) \wedge 3$ else $\times 5[\mathrm{i}]<-0$

fit3B<-glm(ymatB mesB+x4+x5+offset(offsett), family=binomial)

vetor $3 B<-\operatorname{coef}($ fit $3 B)$

summary(fit3B)

Modelo escolhido para o tratamento B : cubico-linear "spline"

\#\#\#\#\# calculo do risco

$\mathrm{x}<-\left(-\right.$ vetor3B[1]-vetor $3 \mathrm{~B}[2]^{*}$ mesB-vetor $3 \mathrm{~B}[3]^{*} \mathrm{x} 4$-vetor3B $[4]^{*} \mathrm{x} 5$ )

$\mathrm{x}<-\exp (\mathrm{x})$

h3B $<-1 /(1+x)$

\#\#\#\#\# cálculo do desvio-padrão da função de risco e intervalo de confiança de $95 \%$

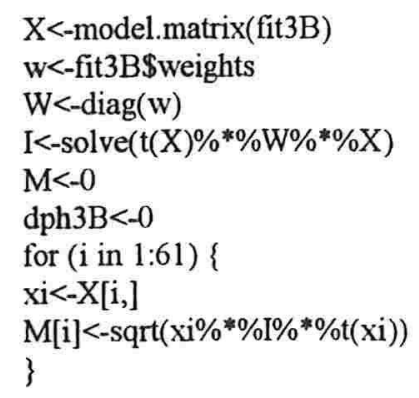


$\mathrm{M}<-\operatorname{diag}(\mathrm{M})$

dph3B $<-(\mathrm{h} 3 \mathrm{~B} \% * \% \operatorname{diag}(1-\mathrm{h} 3 \mathrm{~B})) \% * \% \mathrm{M}$

lih3B $<$-h3B-1.96*dph3B

1 sh $3 \mathrm{~B}<-\mathrm{h} 3 \mathrm{~B}+1.96 * \mathrm{dph} 3 \mathrm{~B}$

\#\#\#\#\# calculo da funcao de sobrevivencia

$\mathrm{h}<-1-\mathrm{h} 3 \mathrm{~B}$

G3B<-cumprod(h)

\#\#\#\#\#\# calculo do desvio da função de sobrevivência e intervalo de confiança de $95 \%$

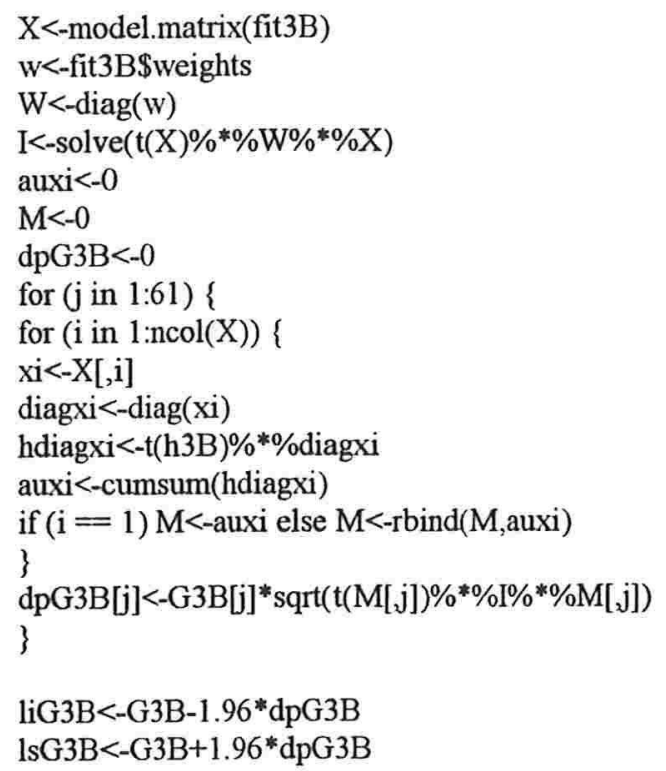

\section{Gráficos}

\#\#\#\#\# Gráfico comum da taxa de risco dos tratamento A e B para o modelo cúbico-linear

win.printer(file="c:Ilcarinelltesellgraficollgraph2_1.wmf", format ="placeable metafile" )

plot(mesA,h3A,type="l",bty="l", xlab="tempo (meses)",ylab="taxa de risco", xlim=c(0,80), ylim=c $(0,0.15)$, cex=1.09)

lines(mesB,h3B, lty $=2$, cex $=1.09$ )

legend(25,0.12,c("Tratamento A", "Tratamento B"),lty=c(1,2),bty="n", cex=1.09)

dev.off()

\#\#\#\#\# Gráfico comum da curva de sobrevida dos tratamento A e B para o modelo cúbico-linear

win.printer(file="c: Ilcarinelltesellgraficollgraph2_2.wmf", format ="placeable metafile")

plot(mesA,G3A,type="l",bty="l",xlab="tempo (meses)",ylab="Prob(sobrevida)",xlim=c(0,80), cex=1.09)

lines(mesB, G3B, lty $=2$, cex $=1.09$ )

legend(30,0.7,c("Tratamento A", "Tratamento B"),lty=c(1,2),bty="n",cex=1.09)

dev.off()

\#\#\#\#\# Gráfico da taxa de risco para o tratamento A com banda de confiança de $95 \%$ referente ao modelo cubico-linear

win.printer(file="c:Ilcarinelltesellgraficollgraph2_3.wmf", format $=$ "placeable metafile" )

plot(mesA,h3A,type="l", bty="l", xlab="tempo (meses)",ylab="taxa de risco",ylim=c(0,0.2),cex=1.09)

lines(mes $A, \operatorname{lih} 3 \mathrm{~A}, 1 \mathrm{ty}=2, \mathrm{cex}=1.09)$

lines(mesA,lsh $3 A, 1 t y=2, \operatorname{cex}=1.09)$

dev.off() 
\#\#\#\# Gráfico da taxa de risco para o tratamento B com banda de confiança de $95 \%$ referente ao modelo cubico-linear

win.printer(file="c:Ilcarinelltesellgraficollgraph2_4. wmf", format $=$ "placeable metafile" $)$

plot(mesB,h3B,type="l",bty="l", xlab="tempo (meses)",ylab="taxa de risco",ylim=c(0,0.2), cex=1.09)

lines(mesB,lih3B, lty $=2$, cex=1.09)

lines (mesB, $1 \operatorname{sh} 3 B, 1$ ty $=2, \operatorname{cex}=1.09$ )

dev.off()

\#\#\# Gráfico da curva de sobrevida para o tratamento A com banda de confiança de $95 \%$ referente ao modelo cubico-linear

win.printer(file="c:Icarinelltesellgraficollgraph2_5wmf", format ="placeable metafile")

plot(mesA,G3A,type="l",bty="l", xlab="tempo (meses)", ylab="Prob(sobrevida)", ylim=c(0,1.0),cex=1.09)

lines(mesA,liG3A, lty=2,cex=1.09)

lines(mesA, $1 \mathrm{sG} 3 \mathrm{~A}, 1 \mathrm{ty}=2, \operatorname{cex}=1.09$ )

dev.off()

\#\#\# Gráfico da curva de sobrevida para o tratamento B com banda de confiança de $95 \%$ referente ao modelo cubico-linear

win.printer(file="c:llcarinelltesellgraficollgraph2_6wmf", format $=$ "placeable metafile")

plot(mesB,G3B,type="l",bty="l", xlab="tempo (meses)", ylab="Prob(sobrevida)", ylim=c(0,1.0),cex=1.09)

lines(mesB,liG3B, lty $=2, \mathrm{cex}=1.09$ )

lines(mesB, lsG3B, lty $=2, \operatorname{cex}=1.09$ )

dev.off()

Análise de resíduos

\#\#\#\#\# Analise de resíduo do modelo cúbico-linear ajustado para o tratamento A

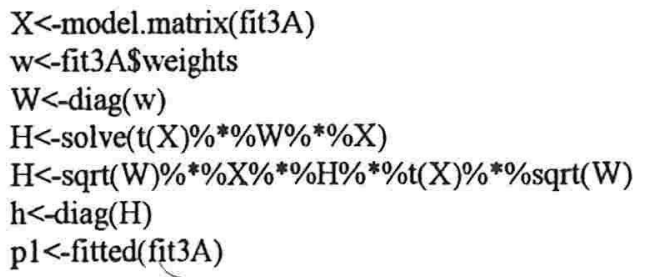

\#\#\#\#\# Gráfico do residuo Tdi versus valores ajustados das probabilidades

rd<-resid(fit3A,type="deviance")

td<-rd*sqrt(1/(1-h))

win.printer(file="c:Icarinelltesellgraficollgraph2_7.wmf", format ="placeable metafile")

plot(p1,td, xlab="Valores ajustados das probabilidades", ylab="Componente do Desvio", cex=1.09)

abline $(-2,0,1$ ty $=2$, cex $=1.09)$

abline $(2,0,1 \mathrm{ty}=2$, cex $=1.09)$

abline $(0,0,1$ ty $=2$, cex $=1.09)$

identify $(\mathrm{pl}, \mathrm{td}, \mathrm{n}=3, \mathrm{cex}=1.09)$

dev.off()

\#\#\#\#\# Grafico da distância de Cook, Ldi, versus valores ajustados das probabilidades

$\mathrm{LDi}<-\left(\mathrm{h}^{*}\left(\mathrm{yA}-\mathrm{nA}{ }^{*} \mathrm{pl}\right)^{\wedge} 2\right) /\left((1-\mathrm{h})^{\wedge} 2^{*} \mathrm{nA}^{*} \mathrm{pl}^{*}(1-\mathrm{pl})\right)$

win.printer(file="c:Ilcarinelltesellgraficollgraph2_8.wmf", format ="placeable metafile")

plot(p1,LDi,xlab="Valores ajustados das probabilidades", ylab="Distância de Cook", cex=1.09)

identify(pl, LDi ,n=2, cex=1.09) 
dev.off()

\#\#\#\#\# Grafico dos residuos padronizados e envelopes

fit.model<-fit3A

$\mathrm{n}<-\mathrm{nA}$

$\mathrm{y}<-\mathrm{yA}$

win. printer(file="c:Ilcarinelltesellgraficollgraph2_9.wmf", format ="placeable metafile" $)$

source("c:Ilcarinellpos/lmod_reg|lcap2llenvelr.bin")

dev.off()

\#\#\#\#\# Analise de residuo do modelo cúbico-linear ajustado para o tratamento B

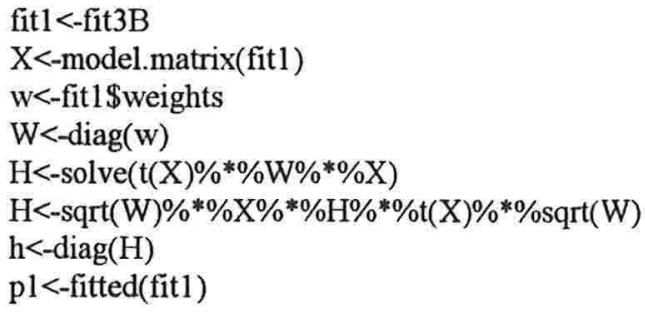

\#\#\#\#\# Gráfico do residuo Tdi versus valores ajustados das probabilidades

win.printer(file="c: llcarinelltesellgraficollgraf2_10.wmf", format ="placeable metafile") rd<-resid(fit1,type="deviance")

td<-rd*sqrt(1/(1-h))

plot $(\mathrm{pl}$,td, xlab="Valores ajustados das probabilidades", ylab="Componente do Desvio",cex=1.09,ylim=c(-2.1,2.1))

abline $(-2,0,1$ ty $=2$, cex $=1.09)$

abline $(2,0,1$ ty $=2$, cex $=1.09)$

abline $(0,0,1$ ty $=2$, cex $=1.09)$

dev.off()

\#\#\#\#\# Grafico da distância de Cook, Ldi, versus valores ajustados das probabilidades

$\mathrm{LDi}<-\left(\mathrm{h}^{*}(\mathrm{yB}-\mathrm{nB} * \mathrm{p} 1)^{\wedge} 2\right) /\left((1-\mathrm{h})^{\wedge} 2 * \mathrm{nB}^{*} \mathrm{pl} \mathrm{p}^{*}(1-\mathrm{p} 1)\right)$

win.printer(file="c: Ilcarinelltesellgraficollgraf2_11.wmf", format ="placeable metafile")

plot(p1,LDi,xlab="Valores ajustados das probabilidades", ylab="Distância de Cook", cex=1.09,ylim=c(0,1.2)) dev.off()

\#\#\#\#\# Grafico dos residuos padronizados e envelopes

$\mathrm{n}<-\mathrm{nB}$

$\mathrm{y}<-\mathrm{yB}$

fit.model<-fit3B

win.printer(file="c:Ilcarinelltesellgraficollgraf2_12.wmf", format ="placeable metafile")

source("c:Ilcarinellposilmod_regllcap2llenvelr.bin")

dev.off() 
Cálculo da estatística de Hosmer e Lemeshow (exemplo)

\section{\#\#\#\# TRATAMENTO A}

\section{p $<$-fitted(fitlA)}

Maux<-cbind(p,yA,nA)

Maux<-Maux[ sort.list(Maux[,1]),]

p<-Maux[,1]

$\mathrm{s}<-$ Maux[,2]

$\mathrm{n}<-$ Maux[,3]

$\mathrm{n} 1<-0$

$\mathrm{n} 2<-0$

n3 $3<-0$

$\mathrm{n} 4<-0$

n $5<-0$

n6 $<-0$

$\mathrm{n} 7<-0$

$\mathrm{n} 8<-0$

$\mathrm{n} 9<-0$

$\mathrm{s} 1<-0$

s2 $<-0$

s $3<-0$

$s 4<-0$

s $5<-0$

s6 $<-0$

s7 $<-0$

s8 $<-0$

s $9<-0$

g1 $<-0$

g2 $<-0$

g $3<-0$

g $4<-0$

g $5<-0$

g6 $<-0$

g7 $<-0$

g8 $<-0$

g $9<-0$

for(i in 1:5)

\{

$\mathrm{nl}[\mathrm{i}]<-\mathrm{n}[\mathrm{i}]$

s1[i]<-s[i]

g1[i]<-p[i]

$\mathrm{n} 2[\mathrm{i}]<-\mathrm{n}[\mathrm{i}+5]$

$s 2[i]<-s[i+5]$

g2 $[\mathrm{i}]<-\mathrm{p}[\mathrm{i}+5]$

n3[i]<- n[i+10]

s3[i]<-s[i+10]

g3 $[i]<-p[i+10]$

n4[i] $<-n[i+15]$

$\mathrm{s} 4[\mathrm{i}]<-\mathrm{s}[\mathrm{i}+15]$

$\mathrm{g} 4[\mathrm{i}]<-\mathrm{p}[\mathrm{i}+15]$

$\mathrm{n} 5[\mathrm{i}]<-\mathrm{n}[\mathrm{i}+20]$

$\mathrm{s} 5[\mathrm{i}]<-\mathrm{s}[\mathrm{i}+20]$ 


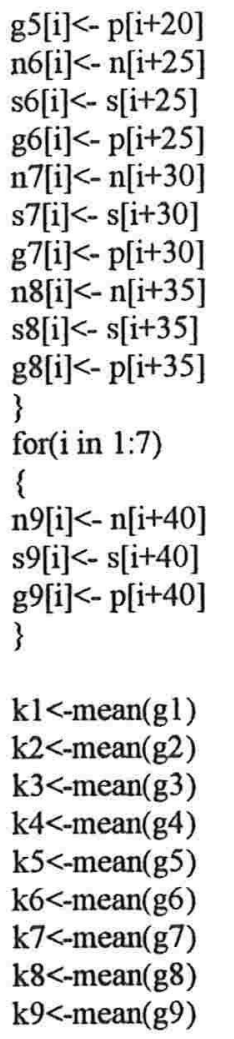

ol<-sum(s1)

$02<-\operatorname{sum}(\mathrm{s} 2)$

03<-sum(s3)

04<-sum(s4)

05<-sum(s5)

06<-sum(s6)

07<-sum(s7)

o8<-sum(s8)

09<-sum(s9)

nn1<-sum(n1)

nn2 $<-\operatorname{sum}(\mathrm{n} 2)$

nn3<-sum(n3)

nn4<-sum(n4)

nn5<-sum(n5)

nn6<-sum(n6)

nn7<-sum(n7)

nn8<-sum(n8)

nn9<-sum(n9)

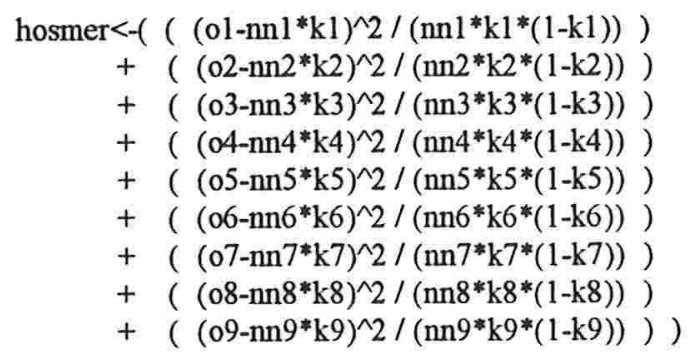

pvalue $<-1$-pchisq(hosmer,7) 


\section{REFERÊNCIAS BIBLIOGRÁFICAS}

Atkinson A. C. (1985). Plots, Transformations and Regressions. Oxford Statistical Science Series, Oxford.

Collet, D. (1991). Modelling Binary Data. Chapman and Hall, London.

DeGroot M. H. (1989). Probability and Statistics. Addison-Wesley, 2ed, London.

Efron, B. (1988). Logistic Regression, Survival Analysis, and the Kaplan-Meier Curve. Journal of the American Statistical Association, 83, 414-425.

Hosmer, D. W. e Lemeshow, S. (1989). Applied Logistic Regression. John Wiley, New York.

James, B. R.(1981). Probabilidade: um curso em nível intermediário. IMPA, Rio de Janeiro.

Lawless J. F. (1982). Statistical Models and Methods for Lifetime Data. John Wiley, New York.

Lee E.T. (1980). Statistical Methods for Survival Data Analysis. Lifetime Learning Publication, Belmont, California.

Lima, A. C. P. (1996). MAE783-Análise de sobrevivência. Curso ministrado no Instituto de Matemática e Estatística da USP, São Paulo. 
Lima, A. C. P. (1988). Estimação em modelos de sobrevivência. Dissertação de Mestrado, IME-USP, São Paulo.

MacCullagh, P. e Nelder, J. A. (1989). Generalized Linear Models. 2ed. Chapman and Hall, London.

Mantel, N. (1966). Evaluation of survival data and two new rank order statistics arising in its consideration. Cancer Chemother, 50, 163-170.

Miller, R. G., Jr. (1981).Survival Analysis. John Wiley, New York.

Miller, R. G., Jr. (1983).'What Price Kaplan-Meier ?". Biometrics, 39, 1077-1081.

Morgan, B. J. T. (1992) Analysis of Quantal Response Data. Chapman and Hall, London.

Paula, G. A., Barbosa, L. S. e Ferreira, R. F. G. (1989). Relatório de Análise Estatística sobre o Projeto: Comportamento biológico evolutivo do tumor $K B$ no decorrer de suas passagens seriadas em ratos nudes adultos. RAE-SEA-8904, IME-USP

Paula, G. A.(1997). Modelos de Regressão. IME-USP, São Paulo (versão preliminar).

Singer J. D. e Willett J. B. (1995). It's about time: Using Discrete-time Survival Analysis to Study Duration and the Timing Events. The Journal of Educational Statistics.

William, D. A. (1986). Generalized linear model diagnostic using deviance and single case deletion. Applied Statistic, 36, 181-191.

William, D. A. (1984). Residuals in generalized linear model. In: Proceedings of the 12th. International Biometrics Conference, Tokyo, 59-68. 